\title{
Tosio Kato's work on non-relativistic quantum mechanics, Part 2
}

\author{
Barry Simon \\ Departments of Mathematics and Physics \\ Mathematics 253-37, California Institute of Technology \\ Pasadena, CA 91125, USA \\ bsimon@caltech.edu
}

Received 8 November 2017

Accepted 20 January 2018

Published 3 April 2019

Communicated by Ari Laptev

\begin{abstract}
We review the work of Tosio Kato on the mathematics of non-relativistic quantum mechanics and some of the research that was motivated by this. Topics in this second part include the absence of embedded eigenvalues, trace class scattering, Kato smoothness, the quantum adiabatic theorem and Kato's ultimate Trotter Product Formula.
\end{abstract}

Keywords: Kato; Schrödinger operators; quantum mechanics.

Mathematics Subject Classification: Primary: 81Q10, 81U05, 47A55; Secondary: 35Q40, 46N50, 81Q15

\section{Eigenvalues, I: Bound State of Atoms}

This is the second part of a two-part article. There is one picture on p. 21 in this part and four pictures in Part 1. The reader should be sure to read the notational warnings near the end of Sec. 1 in Part 1. Just before those warnings is a summary of the organization of the full paper which includes the following about Part 2.

Part 2 begins with two pioneering works on the aspects of bound states - his result on non-existence of positive energy bound states in certain two body systems and his paper on the infinity of bound states for Helium, at least for infinite nuclear mass.

Next, four sections on scattering and spectral theory which discuss the KatoBirman theory (trace class scattering), Kato smoothness, Kato-Kuroda eigenfunction expansions and the Jensen-Kato paper on threshold behavior.

This is an Open Access article published by World Scientific Publishing Company. It is distributed under the terms of the Creative Commons Attribution 4.0 (CC-BY) License. Further distribution of this work is permitted, provided the original work is properly cited. 
Last is a set of three miscellaneous gems: his work on the adiabatic theorem, on the Trotter product formula and his pioneering look at eigenfunction regularity.

In a short companion paper [302] to his famous 1951 paper 301, Kato proved the following theorem.

Theorem 11.1 ([302]). The non-relativistic Helium atom with infinite nuclear mass has infinitely many bound states. With the physical masses, it has at least 25,585 bound states.

The number 25,585 seems unusual but it is just $\sum_{j=1}^{42} j^{2}$ corresponding to the number of bound states in the first 42 complete shells of a Hydrogenic atom.

An operator like the Helium atom, Hamiltonian typically has an essential spectrum, $[\Sigma, \infty)$ (for an arbitrary self-adjoint operator, $A$, we define $\Sigma(A)=\inf \{\lambda \mid \lambda \in$ $\left.\sigma_{\text {ess }}(A)\right\}$, where, we recall, $\sigma_{\text {ess }}(A)=\sigma(A) \backslash \sigma_{d}(A)$ and $\sigma_{d}(A)$, the discrete spectrum, is the isolated points of $\sigma(A)$, the spectrum, for which the spectral projection is finite dimensional (see Sec. 2 in Part 1).

There may be one or more eigenvalues of $A$ below $\Sigma$, i.e. counting multiplicity, $\left\{E_{k}\right\}_{k=1}^{N}, N \in\{0,1,2, \ldots\} \cup\{\infty\}$, where $E_{j-1} \leq E_{j}<\Sigma$. If $N=\infty$, then $\lim _{k \rightarrow \infty} E_{k}=\Sigma$.

Most modern approaches to results like Theorem 11.1 rely on the min-max principle [587, Theorem 3.14.5] which says that if $A$ is self-adjoint and bounded from below, and if one defines

$$
\mu_{n}(A)=\sup _{\psi_{1}, \ldots, \psi_{n-1}}\left(\inf _{\substack{\varphi \in D(A),\|\varphi\|=1 \\ \varphi \perp \psi_{1}, \ldots, \psi_{n-1}}}\langle\varphi, A \varphi\rangle\right)
$$

then $\mu_{j}(A)=E_{j}(A)$ for $j \leq N$ and if $N<\infty$, then for $j>N, \mu_{j}(A)=\Sigma(A)$. Instead, Kato notes the following.

Lemma 11.2. Let $A$ be a self-adjoint operator which is bounded from below and $W \subset D(A)$ a subspace of dimension $k$ so that

$$
\sup _{\varphi \in W,\|\varphi\|=1}\langle\varphi, A \varphi\rangle=J
$$

then

$$
\operatorname{dim} \operatorname{ran} P_{(-\infty, J]}(A) \geq k .
$$

Remarks. (1) $P_{\Omega}(A)$ are the spectral projections of $A$, see [587, Sec. 5.1].

(2) While Kato uses this lemma instead of the min-max principle, it should be emphasized that this lemma can be used to prove that principle!

Proof. Suppose that $\operatorname{dim} \operatorname{ran} P_{(-\infty, J]}(A)<k$. Then we can find $\varphi \in W$ so $\varphi \perp \operatorname{ran} P_{(-\infty, J]}(A)$. Thus, by the spectral theorem, $\langle\varphi, A \varphi\rangle>J$ contrary to (11.2). 
For Kato, $\Sigma$ is defined not in terms of essential spectrum but by

$$
\Sigma=\inf \left\{\lambda \mid \operatorname{dim} \operatorname{ran} P_{(-\infty, \lambda)}(A)=\infty\right\}
$$

although it is the same. His strategy is simple.

(1) Get a lower bound, $\Sigma_{0}$, on $\Sigma$.

(2) Find a $k$-dimensional subspace, $W$, and a $J$ given by (11.2) which obeys $J<\Sigma_{0}$. By (11.4), dim $\operatorname{ran} P_{(\infty, J]}(A)<\infty$ and by the lemma, it is at least $k$ so there must be at least $k$ discrete eigenvalues, counting multiplicity in $(-\infty, J]$.

Let us discuss first the case where the nuclear mass is infinite. The Hamiltonian in suitable units is

$$
H=-\Delta_{1}-\Delta_{2}-\frac{2}{r_{1}}-\frac{2}{r_{2}}+\frac{1}{\left|\boldsymbol{r}_{1}-\boldsymbol{r}_{\mathbf{2}}\right|}
$$

on $L^{2}\left(\mathbb{R}^{6}, d^{6} x\right)$, where $x=\left(\boldsymbol{r}_{\mathbf{1}}, \boldsymbol{r}_{\mathbf{2}}\right), \boldsymbol{r}_{\boldsymbol{j}} \in \mathbb{R}^{3}$. Kato then considers

$$
\tilde{H}=H-\frac{1}{\left|\boldsymbol{r}_{\mathbf{1}}-\boldsymbol{r}_{\mathbf{2}}\right|}=h \otimes \mathbf{1}+\mathbf{1} \otimes h
$$

where

$$
h=-\Delta-\frac{2}{r} .
$$

He talks about "two independent Hydrogen-like atoms" rather than tensor products, but it is the same thing. Thus the spectrum of $\tilde{H}$ is $\left\{\lambda_{1}+\lambda_{2} \mid \lambda_{1}, \lambda_{2} \in \sigma(h)\right\}$. Since $\sigma(h)=\left\{-1 / n^{2}\right\}_{n=1}^{\infty} \cup[0, \infty)$, we see that $\Sigma(\tilde{H})=-1$. Since $H \geq \tilde{H}$, we conclude that

$$
\Sigma(H) \geq-1 \equiv \Sigma_{0}
$$

(we will eventually see that this is actually equality). This concludes Step 1 in this infinite nuclear mass case.

Kato next picked the subspace, $W$, of trial functions. Let $\varphi_{0}$ be the ground state of $h$, i.e.

$$
h \varphi_{0}=-\varphi_{0} .
$$

Kato notes the explicit formula, $\varphi_{0}(\boldsymbol{x})=\pi^{-1 / 2} e^{-|x|}$ but other than that, it is spherically symmetric, the exact formula plays no role. He picks $W=\left\{\varphi_{0} \otimes \eta \mid \eta \in\right.$ $\left.W_{1}\right\}$, where $W_{1}$ will be a suitable subspace of $L^{2}\left(\mathbb{R}^{3}\right)$, i.e. $\varphi\left(\boldsymbol{x}_{\mathbf{1}}, \boldsymbol{x}_{\mathbf{2}}\right)=\varphi_{0}\left(\boldsymbol{x}_{\mathbf{1}}\right) \eta\left(\boldsymbol{x}_{\mathbf{2}}\right)$.

One easily computes that

$$
\langle\varphi, H \varphi\rangle=-1+\langle\eta,(-\Delta+Q(x)) \eta\rangle,
$$

where

$$
Q(x)=-\frac{2}{|x|}+\int\left|\varphi_{0}(y)\right|^{2} \frac{1}{|x-y|} d^{3} y .
$$

The second term in (11.11) is the gravitational potential of a spherically symmetric "mass distribution" $\left|\varphi_{0}(y)\right|^{2} d^{3} y$ and this has been computed by Newton who showed 
that

$$
\int_{S^{2}} \frac{d \omega}{|r \omega-\boldsymbol{x}|}=\frac{1}{\max (|x|, r)},
$$

(where $d \omega$ is normalized measure on the unit 2-sphere). Thus

$$
\begin{aligned}
Q(x) & =-\frac{2}{|x|}+\int\left|\varphi_{0}(y)\right|^{2} \frac{1}{\max (|x|,|y|)} d^{3} y \\
& \leq-\frac{1}{|x|}
\end{aligned}
$$

since $\max (|x|,|y|) \geq|x|$. Thus

$$
\langle\varphi, H \varphi\rangle \leq-1+\langle\eta,(-\Delta-1 / r) \eta\rangle .
$$

Picking $\eta$ in the space of dimension $\frac{1}{6} k(k+1)(2 k+1)$ of linear combinations of eigenfunctions of $-\Delta-1 / r$ of energies $\left\{-\frac{1}{4 j^{2}}\right\}_{j=1}^{k}$, we see that the $J$ of (11.2) is $-1-\left(1 / 4 k^{2}\right)<\Sigma_{0}$, so there are infinitely many eigenvalues below $\Sigma_{0}$ (which also shows that $\left.\Sigma=\Sigma_{0}\right)$.

If one now considers a nucleus of mass $M$ and electrons of mass $m$, the Hamiltonian with the center of mass motion removed becomes (instead of (11.5))

$$
H=-\Delta_{1}-\Delta_{2}-2 \alpha \nabla_{\mathbf{1}} \cdot \nabla_{\mathbf{2}}-\frac{2}{r_{1}}-\frac{2}{r_{2}}+\frac{1}{\left|\boldsymbol{r}_{\mathbf{1}}-\boldsymbol{r}_{\mathbf{2}}\right|},
$$

where

$$
\alpha=\frac{m}{M+m} .
$$

The extra $2 \alpha \boldsymbol{\nabla}_{\mathbf{1}} \cdot \boldsymbol{\nabla}_{\mathbf{2}}$ term, called the Hughes-Eckart term (after [248]), is present if one uses atomic coordinates, $\mathbf{r}_{j}=\mathbf{x}_{j}-\mathbf{x}_{3} ; j=1,2$, where $\mathbf{x}_{j}$ is the coordinate of electron $j$ and $\mathbf{r}_{3}$ is the nuclear position (we will say a lot about such $N$-body kinematics as follows).

The second step in the proof is unchanged. Since $\left\langle\varphi_{0}, \nabla \varphi_{0}\right\rangle=0$ (by either the reality of $\varphi$ or its spherical symmetry), the Hughes-Eckart terms contribute nothing to the calculation of $\langle\varphi, H \varphi\rangle$ and we get a subspace of trial functions of dimension $\frac{1}{6} k(k+1)(2 k+1)$ with $J_{k}=-1-1 / 4 k^{2}$.

Here is how Kato estimated $\Sigma$ in this case. With $p_{j}=-i \nabla_{j}$, one can write

$$
\boldsymbol{p}_{1}^{2}+\boldsymbol{p}_{2}^{2}+2 \alpha \boldsymbol{p}_{1} \cdot \boldsymbol{p}_{2}=\alpha\left(\boldsymbol{p}_{1}+\boldsymbol{p}_{2}\right)^{2}+(1-\alpha)\left(\boldsymbol{p}_{1}^{2}+\boldsymbol{p}_{2}^{2}\right)
$$

Since $\left|\boldsymbol{r}_{1}-\boldsymbol{r}_{2}\right|^{-1} \geq 0$ and $\alpha\left(\boldsymbol{p}_{1}+\boldsymbol{p}_{2}\right)^{2} \geq 0$, we see that

$$
H \geq(1-\alpha)\left(-\Delta_{1}-\Delta_{2}\right)-\frac{2}{r_{1}}-\frac{2}{r_{2}} \equiv H_{\text {Kato }}
$$

As in the infinite mass case, $H_{\text {Kato }}$ is a sum of independent Hydrogen-like atoms, so one finds that

$$
\Sigma \geq \Sigma_{0}=\Sigma\left(H_{\text {Kato }}\right)=-\frac{1}{1-\alpha}
$$


Putting in the physical value of $\alpha$ (i.e. (11.16) with $M=$ Helium nuclear mass and $m=$ electron mass), one finds that

$$
\Sigma_{0} \geq-1-1 / 4 k^{2} \text { if } k \leq 42,
$$

so Kato concluded that there were at least 42 shells and obtained the number 25,585 of Theorem 11.1.

Remarks. (1) As Kato emphasized, before his work, it was not proven that the Helium Hamiltonian had any bound states!

(2) Kato ignored both spin (the Hamiltonian is spin-independent but each electron has two spin states, so on $L^{2}\left(\mathbb{R}^{3 N} ; \mathbb{C}^{2} \otimes \mathbb{C}^{2}, d^{3 N} x\right)$, there are four times as many states) and statistics (the Pauli principle, which, as interpreted by Fermi and Dirac, says that the total wave function is antisymmetric under interchange of a pair of particles in both spin and space). $H$ is symmetric under interchange of the two electrons in space alone, so its eigenfunctions can be chosen to be either symmetric or antisymmetric under spatial interchange. Kato's trial functions are neither but the lower bound, $N_{\text {Kato }}$ that he gets a lower bound on $N_{S}+N_{A}$, the sum of the spatially symmetric and spatially antisymmetric functions. To get a state totally antisymmetric under interchange of space and spin, each spatially symmetric wave function is multiplied by a spin 0 state (multiplicity 1 ) and each spatially antisymmetric state is multiplied by a spin 1 state (multiplicity $3)$. So taking into account both spin and statistics, the total number of states is $N_{S}+3 N_{A}$, so

$$
N_{S}+N_{A} \leq N_{S}+3 N_{A} \leq 3\left(N_{S}+N_{A}\right)
$$

In particular, $N_{\text {Kato }}$ is a lower bound on $N_{S}+3 N_{A}$, so Kato's estimates are lower bounds even if one properly takes into account spin and statistics.

(3) Even in the infinite mass case, Kato's method does not work for three electron atoms. The problem is with his estimate of $\Sigma$. If one drops the repulsion of electron 3 from both 1 and 2, one gets an independent sum of an ion and a charge 3 Hydrogen-like atom. The bottom of the essential spectrum of such a system is actually twice the ground state energy of two of the charge 3 Hydrogen-like atoms which is below the energy of the ion where one expects (and we actually know) the bottom of the essential spectrum really is.

This completes our description of Kato's paper. To go beyond it, one realizes the weak point of his analysis (as seen in Remark 3 above) is not an efficient way of estimating the bottom of the continuous spectrum. As a preliminary to discussing this bottom, we pause to present some $N$-body kinematics, an issue that already entered when we discussed the Hughes-Eckart term above. We will be more expansive than absolutely necessary, in part, because we will need this when we briefly turn to $N$-body scattering in Secs. 13-15] and, in part, because the elegant formalism, which I learned from Sigalov-Sigal [539] (see also [251]), deserves to be better known. 
Given $N$ particles $\left(\boldsymbol{r}_{1}, \ldots, \boldsymbol{r}_{N}\right)$ with masses $m_{1}, \ldots, m_{N}$, we consider the inner product

$$
\left\langle r^{(1)}, r^{(2)}\right\rangle=\sum_{j=1}^{N} m_{j} \boldsymbol{r}_{j}^{(1)} \cdot \boldsymbol{r}_{j}^{(2)}
$$

on $x$-space, $X=\mathbb{R}^{\nu N}$. This is natural because the free Hamiltonian

$$
H_{0}=-\sum_{j=1}^{N}\left(2 m_{j}\right)^{-1} \Delta_{r_{j}}
$$

is precisely one half the Laplace-Beltrami operator for the Riemann metric associated to (11.22).

We let $X^{*}$ be the dual to $X$, which we think of as momentum space. If $\boldsymbol{p} \in X^{*}$ and $\boldsymbol{x} \in X$, they are paired as

$$
\langle\boldsymbol{p}, \boldsymbol{x}\rangle=\sum_{j=1}^{N} \boldsymbol{p}_{j} \cdot \boldsymbol{x}_{j}
$$

as occurs in the Fourier transform. This induces an inner product on $X^{*}$

$$
\left\langle p^{(1)}, p^{(2)}\right\rangle_{X^{*}}=\sum_{j=1}^{N}\left(m_{j}\right)^{-1} \boldsymbol{p}_{j}^{(\mathbf{1})} \cdot \boldsymbol{p}_{j}^{(\mathbf{2})}
$$

consistent with (11.23).

A coordinate change is associated to a linear basis, $e_{1}, \ldots, e_{N}$ of $\mathbb{R}^{N}$ via

$$
\boldsymbol{\rho}_{j}\left(\boldsymbol{x}_{1}, \ldots, \boldsymbol{x}_{N}\right)=\sum_{r=1}^{N} e_{j r} \boldsymbol{x}_{r} .
$$

(The $e_{j r} \in \mathbb{R}$ and $\boldsymbol{x}_{r} \in \mathbb{R}^{\nu}$.)

To be a trifle pedantic, we note that $X$ and $X^{*}$ depend on $N$ and $\nu$. We will use $Y$ for the case $\nu=1$ so that $X=Y \otimes \mathbb{R}^{\nu}$ and the $X$ inner product is the tensor product of the $Y$ inner product and the Euclidean inner product on $\mathbb{R}^{\nu}$ which we denoted with · in (11.22) and (11.26). Since the $e$ 's act on $Y$, we think of them as lying in $Y^{*}$ (acting isotropically on the $\mathbb{R}^{\nu}$ piece). The dual basis $f_{j}$ is defined by

$$
\left\langle f_{j}, e_{\ell}\right\rangle=\delta_{j \ell}, \quad \text { i.e. } \sum_{r=1}^{N} f_{j r} e_{\ell r}=\delta_{j \ell} \text {. }
$$

If we think of $E, F$ as the $N \times N$ matrices with $F_{j r}=\left(f_{j}\right)_{r}, E_{j r}=\left(e_{j}\right)_{r}$, then (11.27) says that $F E^{T}=\mathbf{1}$. Since $\mathbf{1}^{T}=\mathbf{1}$ and for finite matrices $A B=\mathbf{1} \Rightarrow B A=$ 1, we conclude that $E F^{T}=E^{T} F=F^{T} E=1$, i.e.

$$
\sum_{j} f_{r j} e_{s j}=\sum_{j} f_{j r} e_{j s}=\sum_{j} f_{s j} e_{r j}=\sum_{j} f_{j s} e_{j r}=\delta_{r s} .
$$


First this implies that if

$$
\boldsymbol{k}_{j}\left(\boldsymbol{p}_{1}, \ldots, \boldsymbol{p}_{N}\right)=\sum_{q=1}^{N} f_{j q} \boldsymbol{p}_{q}
$$

then by (11.28)

$$
\sum_{j=1}^{N} \boldsymbol{k}_{j} \cdot \boldsymbol{\rho}_{j}=\sum_{j=1}^{N} \sum_{q=1}^{N} \sum_{r=1}^{N} f_{j q} e_{j r} \boldsymbol{p}_{q} \cdot \boldsymbol{x}_{r}=\sum_{q=1}^{N} \boldsymbol{p}_{q} \cdot \boldsymbol{r}_{q},
$$

so the $k$ 's are the Fourier duals to the $\rho$ 's and (11.28) describes the transformation of momenta.

Moreover, we claim that $\left\langle e_{j}, e_{k}\right\rangle_{Y^{*}}$ and $\left\langle f_{j}, f_{k}\right\rangle_{Y}$ are inverse matrices to each other, i.e.

$$
\langle e, e\rangle_{Y *}\langle f, f\rangle_{Y}=\mathbf{1}
$$

If $e_{j}^{(0)}=\delta_{j}$, then $f_{j}^{0}=\delta_{j}$ and $\left\langle e_{j}^{(0)}, e_{k}^{(0)}\right\rangle_{Y^{*}}=m_{j}^{-1} \delta_{j k}$ is indeed the inverse to $\left\langle f_{j}^{(0)}\right.$, $\left.f_{k}^{(0)}\right\rangle_{Y}=m_{j} \delta_{j k}$. Since $e_{r}=\sum_{q=1}^{N} E_{r q} e_{q}^{(0)}$ and $f_{j}=\sum_{k=1}^{N} F_{j k} f_{K}^{(0)}$, we see that $\langle e, e\rangle_{Y^{*}}\langle f, f\rangle_{Y}=E^{T}\left\langle e^{(0)}, e^{(0)}\right\rangle_{Y^{*}} E F^{T}\left\langle f^{(0)}, f^{(0)}\right\rangle_{Y} F=\mathbf{1}$ by (11.28) and (11.27) for the $e^{(0)}, f^{(0)}$ special case just proven.

Finally by (11.26) and (11.27), we see that

$$
\begin{gathered}
\sum_{j=1}^{N} m_{j} \boldsymbol{r}_{j}^{2}=\sum_{r, s=1}^{N}\left\langle f_{r}, f_{s}\right\rangle_{Y} \boldsymbol{\rho}_{r} \cdot \boldsymbol{\rho}_{s}, \\
\sum_{j=1}^{N} m_{j}^{-1} \boldsymbol{p}_{j}^{2}=\sum_{r, s=1}^{N}\left\langle e_{r}, e_{s}\right\rangle_{Y^{*}} \boldsymbol{k}_{r} \cdot \boldsymbol{k}_{s} .
\end{gathered}
$$

Example 11.3 (Removing the center of mass). First consider $N=2$. Since we have $V\left(\boldsymbol{r}_{1}-\boldsymbol{r}_{2}\right)$, we want $\boldsymbol{r}_{1}-\boldsymbol{r}_{2}$ to be one coordinate, i.e. $e_{1}=(1,-1)$. The natural second coordinate should be orthogonal in $Y^{*}$, i.e. $\frac{1}{m_{1}} e_{21}-\frac{1}{m_{2}} e_{22}=0$ so $\left(m_{1}, m_{2}\right)$ will work but it is more usual to take $e_{2}=\frac{1}{M}\left(m_{1}, m_{2}\right), M=m_{1}+m_{2}$ the total mass. That is, the second coordinate is $\left(m_{1} \boldsymbol{r}_{1}+m_{2} \boldsymbol{r}_{2}\right) / M$, the center of mass. One computes

$$
\begin{aligned}
& \left\langle e_{1}, e_{1}\right\rangle_{Y^{*}}=\frac{1}{m_{1}}+\frac{1}{m_{2}} \equiv \frac{1}{\mu}, \quad\left\langle e_{1}, e_{2}\right\rangle_{Y^{*}}=0, \\
& \left\langle e_{2}, e_{2}\right\rangle_{Y^{*}}=\frac{1}{M^{2}}\left(\frac{m_{1}^{2}}{m_{1}}+\frac{m_{2}^{2}}{m_{2}}\right)=\frac{1}{M} .
\end{aligned}
$$

We compute

$$
f_{1}=\left(\frac{m_{2}}{M},-\frac{m_{2}}{M}\right), \quad f_{2}=(1,1)
$$


By either direct calculation or 11.31,

$$
\begin{aligned}
& \left\langle f_{1}, f_{1}\right\rangle_{Y}=\frac{m_{1} m_{2}^{2}+m_{1}^{2} m_{2}}{M^{2}}=\frac{m_{1} m_{2}}{M}=\mu, \quad\left\langle f_{1}, f_{2}\right\rangle_{Y}=0, \\
& \left\langle f_{2}, f_{2}\right\rangle_{Y}=m_{1}+m_{2}=M .
\end{aligned}
$$

Thus

$$
\begin{aligned}
& \boldsymbol{r}_{12}=\boldsymbol{r}_{1}-\boldsymbol{r}_{2}, \quad \boldsymbol{R}=\frac{1}{M}\left(m_{1} \boldsymbol{r}_{1}+m_{2} \boldsymbol{r}_{2}\right), \\
& \boldsymbol{k}_{12}=\frac{m_{2} \boldsymbol{p}_{1}-m_{1} \boldsymbol{p}_{2}}{M}, \quad \boldsymbol{K}=\boldsymbol{p}_{1}+\boldsymbol{p}_{2}
\end{aligned}
$$

and we see that

$$
m_{1} \boldsymbol{r}_{1}^{2}+m_{2} \boldsymbol{r}_{2}^{2}=\mu \boldsymbol{r}_{12}^{2}+M \boldsymbol{R}^{2} ; \quad H_{0}=-\frac{1}{2 M} \Delta_{\boldsymbol{R}}-\frac{1}{2 \mu} \Delta_{\boldsymbol{r}_{12}} .
$$

For $N$ bodies, motivated by the above, we want to take $f_{N}=(1, \ldots, 1)$ and $f_{1}, \ldots, f_{N-1}$ all orthogonal to it. Then $\langle f, f\rangle_{Y}$ will be the direct sum of an $(N-$ $1) \times(N-1)$ matrix and $\left\langle f_{N}, f_{N}\right\rangle_{Y}=M$. Thus $\langle e, e\rangle_{Y *}$ with be the direct sum of an $(N-1) \times(N-1)$ matrix and $\left\langle e_{N}, e_{N}\right\rangle_{Y^{*}}=1 / M$. Moreover, we claim that

$$
\left\langle e_{N}, f\right\rangle=\left\langle f_{N}, f\right\rangle /\left\langle f_{N}, f_{N}\right\rangle
$$

since this holds for each $f_{j}$. Putting $f=\delta_{j}$, we conclude that $e_{N}=$ $M^{-1}\left(m_{1}, \ldots, m_{N}\right)$. We summarize in this Proposition.

Proposition 11.4. In any coordinate system, $\boldsymbol{\rho}_{1}, \ldots, \boldsymbol{\rho}_{N}$, where $\boldsymbol{\rho}_{j}, j=1, \ldots$, $N-1$ is a linear combination of $\boldsymbol{r}_{k}-\boldsymbol{r}_{\ell}$ and

$$
\boldsymbol{\rho}_{N}=\frac{1}{M} \sum_{j=1}^{N} m_{j} \boldsymbol{r}_{j}
$$

we have that

$$
H_{0}=-\sum_{j=1}^{N} \frac{1}{2 m_{j}} \Delta_{r_{j}}=h_{0} \otimes \mathbf{1}+\mathbf{1} \otimes T_{0}
$$

where $h_{0}=-(2 M)^{-1} \Delta_{\boldsymbol{\rho}_{N}}$ and $T_{0}$ is a quadratic form in $-i \boldsymbol{\nabla}_{\boldsymbol{\rho}_{j}}, j=1, \ldots, N-1$.

Example 11.5 (Atomic coordinates). This is named for the natural coordinates when there is a heavy nucleus, $\boldsymbol{r}_{N}$ and $N-1$ electrons. We take (with $m_{j}=m$ for $j=1, \ldots, N-1)$

$$
\boldsymbol{\rho}_{j}=\boldsymbol{r}_{j}-\boldsymbol{r}_{N}, \quad j=1, \ldots, N-1 ; \quad \boldsymbol{\rho}_{N}=\frac{1}{M} \sum_{j=1}^{N} m_{j} \boldsymbol{r}_{j} .
$$

Thus, by 11.26,

$$
e_{j}=\delta_{j}-\delta_{N} ; \quad e_{N}=\frac{1}{M}
$$


Since $\langle a, a\rangle_{Y^{*}}=\sum_{j=1}^{N} m_{j}^{-1} a_{j}^{2}$, we see that

$$
\begin{aligned}
\left\langle e_{N}, e_{j}\right\rangle_{Y^{*}} & =M^{-1} \delta_{N j}, \\
\left\langle e_{j}, e_{j}\right\rangle_{Y^{*}} & =\frac{1}{m}+\frac{1}{m_{N}} \equiv \frac{1}{\mu}, \quad j=1, \ldots, N-1, \\
\left\langle e_{j}, e_{k}\right\rangle & =\frac{1}{m_{N}}, \quad 1 \leq j, \quad k \leq N-1 ; \quad j \neq k .
\end{aligned}
$$

Thus, by (11.33),

$$
\begin{aligned}
T_{0} & =-\sum_{j, k=1}^{N-1} \frac{1}{2}\left\langle e_{j}, e_{k}\right\rangle_{Y^{*}} \nabla_{j} \cdot \nabla_{k} \\
& =-\sum_{j=1}^{N-1} \frac{1}{2 \mu} \Delta_{j}-\frac{1}{m_{N}} \sum_{j<k} \nabla_{j} \cdot \nabla_{k}
\end{aligned}
$$

(there is no 2 in front of $m_{N}$ because we have changed from a sum over $j \neq k$ to $j \leq k)$. Noting that

$$
\frac{\mu}{m_{N}}=\frac{m m_{n}}{m+m_{n}} \frac{1}{m_{n}}=\frac{m}{m_{n}+m}
$$

which is (11.15) / (11.16) (taking into account a changed meaning for the symbol $M$ there and here!).

Example 11.6 (Jacobi coordinates). These coordinates changes go back to classical mechanics. Jacobi noted that one could avoid cross-terms in the kinetic energy changing first from $r_{1}$ and $r_{2}$ to $r_{1,2}$ and the center of mass, $R_{12}$, of the first two particles. Then one goes from $R_{12}$ and $r_{3}$ to $r_{3}-R_{12}$ and the center of mass of the first three particles. After $N-1$ steps, one has $R$, the total center of mass as one of the coordinates, and $N-1$ "internal" coordinates.

Example 11.7 (Clustered Jacobi coordinates). Given $\{1, \ldots, N\}$, a cluster decomposition or clustering, $\mathcal{C}=\left\{C_{\ell}\right\}_{\ell=1}^{k}$, is a partition, i.e. a family of disjoint subsets whose union is $\{1, \ldots, N\}$. We set $\#\left(C_{\ell}\right)$ to be the number of particles in $C_{\ell}$. A coordinate, $\boldsymbol{\rho}$, is said to be internal to $C_{\ell}$ if it is a function only of $\left\{\boldsymbol{r}_{m}\right\}_{m \in C_{\ell}}$ and is invariant under $\boldsymbol{r}_{m} \rightarrow \boldsymbol{r}_{m}+\boldsymbol{a}$, equivalently, it is a linear combination of $\left\{\boldsymbol{r}_{m}-\boldsymbol{r}_{q}\right\}_{m, q \in C_{\ell}}$. A clustered Jacobi coordinate system is a set of $\#\left(C_{\ell}\right)-1$ independent internal coordinates for each cluster together with $\boldsymbol{R}_{\ell}=\left(\sum_{q \in C_{\ell}} m_{q} \boldsymbol{r}_{q}\right) /\left(\sum_{q \in C_{\ell}} m_{q}\right)$. If we write $\mathcal{H}\left(C_{\ell}\right)$ to be $L^{2}$ of the internal coordinates and $\mathcal{H}^{(\mathcal{C})}$ to be $L^{2}$ of the internal coordinates, then

$$
\begin{aligned}
\mathcal{H} & =\mathcal{H}^{(\mathcal{C})} \otimes \bigotimes_{\ell=1}^{k} \mathcal{H}\left(C_{\ell}\right), \\
H_{0} & =\widetilde{T}^{(\mathcal{C})} \otimes \mathbf{1} \cdots \otimes \mathbf{1}+\sum_{\ell=1}^{k} \mathbf{1} \otimes \cdots \otimes T\left(C_{\ell}\right) \otimes \cdots \otimes \mathbf{1},
\end{aligned}
$$




\section{B. Simon}

where $\widetilde{T}^{(\mathcal{C})}=-\sum_{\ell=1}^{k}\left(2 M\left(C_{\ell}\right)\right)^{-1} \Delta_{\boldsymbol{R}_{\ell}}$ and $T\left(C_{\ell}\right)$ is a quadratic form in the derivatives of the internal coordinates.

As noted, the big limitation in Kato's work on Helium bound states concerns his estimate of $\Sigma$, the bottom of the essential spectrum of $H$. We turn to understanding that. In the two-body case, $H=-\Delta+V$, one expects that $\sigma_{\text {ess }}(H)=[0, \infty)$. This requires that $V$ goes to zero at spatial infinity in some sense. If one is looking at $V$ 's for which $D(H)=D(-\Delta)$, the natural condition is that $V(-\Delta+1)^{-1}$ is a compact operator (see [587, Sec. 3.14]). To be explicit, we introduce $L^{p}\left(\mathbb{R}^{\nu}\right)+L^{\infty}\left(\mathbb{R}^{\nu}\right)_{\epsilon}$ to be the set of $V$ so that for any $\epsilon>0$, one can decompose $V=V_{1, \epsilon}+V_{2, \epsilon}$ with $V_{1, \epsilon} \in L^{p}\left(\mathbb{R}^{\nu}\right)$ and $\left\|V_{2, \epsilon}\right\|_{\infty} \leq \epsilon$. If $p$ is $\nu$-canonical, one can prove that if $V \in L^{p}\left(\mathbb{R}^{\nu}\right)+L^{\infty}\left(\mathbb{R}^{\nu}\right)_{\epsilon}$, then $V(-\Delta+1)^{-1}$ is compact and $\sigma_{\text {ess }}(H)=[0, \infty)$. If one wishes, there are Stummel-type conditions to replace this, but we will make such $L^{p}$ assumptions below for simplicity of exposition.

We also want to remove the total center of mass motion if all masses are finite. That is, we let $\boldsymbol{R}=\left(\sum_{j=1}^{N} m_{j} \boldsymbol{r}_{j}\right) /\left(\sum_{j=1}^{N} m_{j}\right)$ and pick some set of internal coordinates so that $\mathcal{H}^{\text {full }}=\mathcal{H}_{\mathrm{CM}} \otimes \mathcal{H}, \mathcal{H}^{\text {full }}=L^{2}\left(\mathbb{R}^{\nu N}\right), \mathcal{H}_{\mathrm{CM}}=$ functions of $\boldsymbol{R}$, $\mathcal{H}=$ functions of the internal coordinates. If $H^{\text {full }}=H_{0}+\sum_{j<k} V_{j k}$, then under this tensor product decomposition

$$
H^{\text {full }}=H_{0, \mathrm{CM}} \otimes \mathbf{1}+\mathbf{1} \otimes H,
$$

where $H_{0, \mathrm{CM}}=-\left(2 \sum_{j=1}^{N} m_{j}\right)^{-1} \Delta_{\boldsymbol{R}}$. We will consider $H$ as follows.

In (11.50), the operator $\widetilde{T}^{(\mathcal{C})}$ has a decomposition like (11.51), where $\mathcal{H}$ is replaced by $\mathcal{H}^{(\mathcal{C})}$, the functions of the differences of the centers of mass of the $C_{j}$. We write

$$
\widetilde{T}^{(\mathcal{C})}=H_{0, \mathrm{CM}} \otimes \mathbf{1}+\mathbf{1} \otimes T^{(\mathcal{C})} .
$$

Given a cluster decomposition, $\mathcal{C}=\left\{C_{\ell}\right\}_{\ell=1}^{k}$, we write $(j q) \subset \mathcal{C}$ if $j$ and $q$ are in the same cluster of $\mathcal{C}$ and $(j q) \not \subset \mathcal{C}$ if they are in different clusters. We define

$$
\begin{aligned}
V\left(C_{\ell}\right) & =\sum_{\substack{j, q \in C_{\ell} \\
j<q}} V_{j q}, \\
V(\mathcal{C}) & =\sum_{\ell=1}^{k} V\left(C_{\ell}\right)=\sum_{\substack{(j q) \subset \mathcal{C} \\
j<q}} V_{j q}, \\
I(\mathcal{C}) & =\sum_{j<q} V_{j q}-V(\mathcal{C})=\sum_{\substack{(j q) \not \subset \mathcal{C} \\
j<q}} V_{j q} .
\end{aligned}
$$

$V(\mathcal{C})$ is the intracluster interaction and $I(\mathcal{C})$ the intercluster interaction. We define on $\mathcal{H}\left(C_{\ell}\right)$

$$
h\left(C_{\ell}\right)=T\left(C_{\ell}\right)+V\left(C_{\ell}\right) .
$$




$$
\begin{aligned}
H(\mathcal{C}) & =T^{(\mathcal{C})} \otimes \mathbf{1} \cdots \otimes \mathbf{1}+\sum_{\ell=1}^{k} \mathbf{1} \otimes \cdots \otimes h\left(C_{\ell}\right) \otimes \cdots \otimes \mathbf{1} \\
& =H-I(\mathcal{C}), \\
\Sigma(\mathcal{C}) & =\sum_{\ell=1}^{k} \inf \sigma\left(H\left(C_{\ell}\right)\right) .
\end{aligned}
$$

We let $\mathcal{C}_{\text {min }}$ be the one cluster decomposition of $\{1, \ldots, N\}$ so $H\left(\mathcal{C}_{\text {min }}\right)=H$. We note that

$$
\mathcal{C} \neq \mathcal{C}_{\min } \Rightarrow \sigma\left(T^{(\mathcal{C})}\right)=[0, \infty) .
$$

By 11.57, we have that $\sigma(H(\mathcal{C}))=\sigma\left(T^{(\mathcal{C})}\right)+\sigma\left(H\left(C_{1}\right)\right)+\cdots+\sigma\left(H\left(C_{k}\right)\right)$. By (11.59),

$$
\mathcal{C} \neq \mathcal{C}_{\min } \Rightarrow \sigma(H(\mathcal{C}))=[\Sigma(\mathcal{C}), \infty)
$$

When we discuss $N$-body spectral and scattering theory briefly in Secs. 12 14, we will be interested in thresholds. A threshold, $t$, is a decomposition $\mathcal{C}=\left\{C_{\ell}\right\}_{\ell=1}^{k} \neq$ $\mathcal{C}_{\min }$ and an eigenvalue, $E_{\ell}$ of $h\left(C_{\ell}\right)$ for each $\ell=1, \ldots, k$. The threshold energy is $E(t)=\sum_{\ell=1}^{k} E_{\ell}$. Of course, $E(t) \geq \Sigma(\mathcal{C})$.

Fix $\mathcal{C} \neq \mathcal{C}_{\text {min }}$. Pick distinct vectors, $X_{1}, \ldots, X_{k} \in \mathbb{R}^{\nu}$. For $\lambda \in \mathbb{R}$, let $U(\lambda)$ be the unitary implementing $x_{j} \mapsto x_{j}+\lambda X_{p}$ if $j \in C_{q}$. It is easy to see that $U(\lambda) H(\mathcal{C}) U(\lambda)^{-1}=H(\mathcal{C})$ and if each $V_{j q} \in L^{p}\left(\mathbb{R}^{\nu}\right)+L^{\infty}\left(\mathbb{R}^{\nu}\right)_{\epsilon}$, then for all $\varphi \in$ $D(-\Delta)$, one has that

$$
\lim _{\lambda \rightarrow \infty}\left[U(\lambda) H U(\lambda)^{-1}-H(\mathcal{C})\right] \varphi=0
$$

which implies [587, Problem 3.14.5] that $\sigma(H(\mathcal{C}))=[\Sigma(\mathcal{C}), \infty) \subset \sigma(H)$. In particular, if

$$
\Sigma=\inf _{\mathcal{C} \neq \mathcal{C}_{\min }} \Sigma(\mathcal{C})
$$

then

$$
[\Sigma, \infty) \subset \sigma(H)
$$

The celebrated HVZ theorem says that.

Theorem 11.8 (HVZ Theorem). For $N$-body Hamiltonians with $V_{j q} \in L^{p}\left(\mathbb{R}^{\nu}\right)+$ $L^{\infty}\left(\mathbb{R}^{\nu}\right)_{\epsilon}$ (with $p \nu$-canonical), one has that

$$
\sigma_{\mathrm{ess}}(H)=[\Sigma, \infty) .
$$

Remarks . (1) There is a variant where there are infinite mass particles, i.e. some $V_{j}$ terms, and the center of mass is not removed. Decompositions are now of $\{0,1, \ldots, N\}$. One says that $(j) \subset \mathcal{C}$ if 0 and $j$ are in the same cluster.

(2) The result is named after Hunziker [249, van Winter 628, and Zhislin 676]. 
(3) There are essentially three generations of proofs of this theorem. The initial proofs of Hunziker and van Winter relied on integral equations (what are now called the Weinberg-van Winter equations). van Winter restricted her work to $L^{2}\left(\mathbb{R}^{3}\right)$ potentials since she only considered Hilbert-Schmidt operators while Hunziker's independent work handled the general case above. This work was independent of the earlier work of Zhislin who only considered and proved results for atomic Hamiltonians. His methods were geometric.

(4) The second wave concerns geometric proofs by Enss [135], Simon [556, Agmon [5], Gårding [176] and Sigal [527]. In one variant, the key is a geometric fact that there exists a partition of unity $\left\{J_{\mathcal{C}}\right\}_{\mathcal{C} \neq \mathcal{C}_{\min }}$ indexed non-minimal partitions so that $\sum_{\mathcal{C}} J_{\mathcal{C}}=1$ and so that on $\operatorname{supp} J_{\mathcal{C}} \cap\{x|| x \mid>1\}$, one has that, for some $Q>0$, $\left|x_{j}-x_{k}\right| \geq Q|x|$ if $(j k) \not \subset \mathcal{C}$. One proves that $[f(H)-f(H(\mathcal{C}))] J_{\mathcal{C}}$ is a compact operator for continuous functions, $f$ of compact support. This, in turn, implies that when supp $f \subset(-\infty, \Sigma)$, then $f(H)$ is compact. For details, see [98, Sec. 3.3]. Agmon's version [5] looks at limits as one translates in an arbitrary direction and is especially intuitive. In this regard, Agmon considered a class of potentials that generalize $N$-body systems. $\left\{\pi_{j}\right\}$ is a family of nontrivial projections in $\mathbb{R}^{\nu N}$ and $V=\sum V_{j}\left(\pi_{j} x\right)$, where $V_{j}$ is a functions on $\mathbb{R}^{\operatorname{dim} \operatorname{ran} \pi_{j}}$. This setup has been used by many authors since.

(5) The third generation works in cases where $\sigma_{\text {ess }}(A)$ can have gaps. This approach appeared (more or less independently) in [83, 84, 180, 394, 395, 423, 471. Perhaps, the cleanest result from 395] defines the notion of right limits and proves that $\sigma_{\text {ess }}(H)$ is the union over all right limits of $\sigma\left(H_{r}\right)$. See also [581 Sec. 7.2].

With the HVZ theorem in hand, one can easily carry Kato's argument to its logical conclusion.

Theorem 11.9 ([542]). Let $H$ be an $N$-body Hamiltonian with center of mass removed. Suppose that $\Sigma$ is a two-body threshold, i.e. there is a cluster decomposition, $\mathcal{C}=\left\{C_{1}, C_{2}\right\}$ and vectors, $\varphi_{j} \in \mathcal{H}\left(C_{j}\right), j=1,2$ so that $H\left(C_{j}\right) \varphi_{j}=$ $E_{j} \varphi_{j},\left\|\varphi_{j}\right\|=1$ and $E_{1}+E_{2}=\Sigma$. Define $W$ on $\mathbb{R}^{\nu}$ as follows: $y \in \mathbb{R}^{\nu}$ is the difference of the centers of mass of $C_{1}$ and $C_{2}$ and let $x_{k}\left(y, \zeta_{1}, \zeta_{2}\right)$ be the position of particle $k$ in terms of $y$ and the internal coordinates $\zeta_{j}$ of $C_{j}$. Then

$$
W(y)=\sum_{\substack{q \in C_{1} \\ k \in C_{2}}} \int V_{q k}\left(x_{q}\left(y, \zeta_{j}\right)-x_{k}\left(y, \zeta_{j}\right)\right)\left|\varphi_{1}\left(\zeta_{1}\right)\right|^{2}\left|\varphi_{2}\left(\zeta_{2}\right)\right|^{2} d \zeta_{1} d \zeta_{2} .
$$

Let $\mu$ be the reduced mass of the two clusters and suppose that

$$
-(2 \mu)^{-1} \Delta_{y}+W(y)
$$

has an infinite number of eigenvalues below 0 as an operator on $L^{2}\left(\mathbb{R}^{\nu}\right)$. Then $H$ has an infinite number of eigenvalues below $\Sigma$.

Remarks. (1) Thus, with $M\left(C_{j}\right)=\sum_{k \in C_{j}} m_{k}$, we have that $\mu^{-1}=M\left(C_{1}\right)^{-1}+$ $M\left(C_{2}\right)^{-1}$. 
(2) One might think that if $j \in C_{1}$, then $x_{j}\left(y, \zeta_{1}, \zeta_{2}\right)$ is independent of $\zeta_{2}$ but that is wrong for the total center of mass, $\boldsymbol{R}$, enters in $x_{j}$ and that causes a $\zeta_{2}$ dependence.

(3) The proof is essentially unchanged from the ideas in Kato 302. If $\psi\left(y, \zeta_{1}, \zeta_{2}\right)=\varphi_{1}\left(\zeta_{1}\right) \varphi_{2}\left(\zeta_{2}\right) \eta(y)$, then $\langle\psi, H \psi\rangle=\Sigma+\left\langle\eta,\left(-(2 \mu)^{-1} \Delta+W\right) \eta\right\rangle$.

(4) This result is from Simon [542] who revisited Kato's paper after the discovery of the HVZ theorem.

Now fix $Z, N>0 . N$ is an integer but $Z$ need not be. We define on $L^{2}\left(\mathbb{R}^{3 N}\right)$ :

$$
\begin{aligned}
& H(Z, N)=\sum_{j=1}^{N}\left(-\Delta_{j}-\frac{Z}{\left|x_{j}\right|}\right)+\sum_{1 \leq j, k \leq N} \frac{1}{\left|x_{j}-x_{k}\right|}, \\
& E(Z, N)=\inf \sigma(H(Z, N)) .
\end{aligned}
$$

One can accommodate Hughes-Eckart terms in much of the discussion but we will not include them.

By the arguments before (11.60), $\sigma(H(Z, N-1)) \subset \sigma(H(Z, N))$ so the HVZ theorem implies that

$$
\Sigma(H(Z, N))=E(Z, N-1)
$$

so we are interested in

$$
\delta(Z, N)=-E(Z, N)+E(Z, N-1),
$$

the ionization energy to remove electron $N$ from a nucleus of charge $Z$. Put differently, $\delta \geq 0$ and $\delta>0$ if and only if $N$ electrons bind to a charge $Z$ nucleus.

Corollary 11.10 ([676]). If $Z>N-1$, then $H(Z, N)$ has infinitely many bound states below $\Sigma$. In particular, $\delta(Z, N)>0$.

Remarks. (1) This is because by induction, $\Sigma$ is determined by a two-cluster breakup into $N-1$ particles (in the same cluster as 0 ) and one particle and then that $W(y)=[Z-(N-1)]|y|^{-1}+\mathrm{o}(1 /|y|)$ and such a potential has infinitely many bound states.

(2) This result was first proved by Zhislin using arguments somewhat more involved than Kato's argument (and before Simon noted that Kato's arguments work).

This completes the summary of the direct extensions of Kato's work. We will end this section with a brief discussion of results on bound states of $H(Z, N)$ which are a direct descendent of Kato's consideration. There is an enormous literature not only on this subject but also on bounds on the number of bound states when finite and on moments of the eigenvalues. We refer the reader to the forthcoming book of Frank et al. [155].

The other side of Corollary 11.10 is as follows.

Theorem 11.11. If $Z \leq N-1$, then $H(Z, N)$ has only finitely many bound states. 
Remarks. (1) This theorem is due to Zhislin 677. There were earlier results of Uchiyama 625. (for $N=2, Z<1$ ), and by Vugal'ter-Zhislin 633. and Yafaev 660, 661] (for $Z=N-1$ ).

(2) The intuition is that the leftover Coulomb repulsion (if $Z<N-1$ ) or residual Coulomb attraction (if $Z=N-1$ ) is such that an effective $-\Delta+W$ has only finitely many states. Of course, one needs techniques to conclude that when an effective two-body problem has that property, the full $N$-body does - one of the most effective methods is due to Sigal [527]. I note in passing that there are three particle systems with short-range interactions that surprisingly have an infinite number of bound states, $\left\{E_{j}\right\}_{j=1}^{\infty}$ with asymptotic geometric sequence placement, i.e. $E_{j+1} / E_{j} \rightarrow \alpha<1$. At least two of the three two body clusters must have zero energy resonances (what this means is discussed in Sec. 16) so the bottom of the essential spectrum is 0. The discovery on a formal level is due to Efimov [132] after whom the effect is named. For mathematical proofs, see 659, 604, 605, 592, 455. Wang [636, 637] discussed this for $N$-body systems. For popular science treatments of experimental verification of the geometric progression (even for small $j$ !) see [454, 652.

(3) This theorem is stated for systems with no statistics. For $Z<N-1$, the result extends without much trouble to Fermi statistics [677]. For $Z=N-1$, one needs to assume that there is not an atomic ground state with a dipole moment (for there to be such a state, there would need to be a degeneracy of states with different parity) because $-\Delta+\lambda \hat{e} \cdot \boldsymbol{r} /(1+r)^{3}$ has an infinity of bound states when $\lambda$ is large enough. In fact, in 556, it is claimed (quoting Lieb) that a molecule with two centers, $Z_{1}=1 / 3, Z_{2}=2 / 3, N=2$ (so $Z=N-1$ ) and $\left|\boldsymbol{R}_{\mathbf{1}}-\boldsymbol{R}_{\mathbf{2}}\right|$ large will have an infinity of bound states (although a proof has never been published to my knowledge). In any event, under an assumption about no atomic ground state with dipole moment, the theorem does extend to $N=Z+1633$.

For most of the discussion as follows, we look at $E(Z, N)$ with Fermi statistics. One might expect that for $Z$ fixed, one has that $\delta(Z, N)=0$ for all sufficiently large $N$, i.e. there is an $N_{c}(Z)$ so that $\delta(Z, N)=0$ if $N \geq N_{c}(Z)$ and so that $\delta\left(Z, N_{c}(Z)-1\right)>0$. Ruskai [506] and Sigal [527, 529] proved that for every $Z$, there is such an $N_{c}(Z)$ and Lieb [410] found a simple, elegant argument that $N_{c}(Z) \leq 2 Z+1$ which, in particular, implies that $H^{--}$does not exist although $H^{-}$ does.

In nature, there is no known example for $\delta(Z, N)>0$ if $N \geq Z+2$, that is, there are once negatively charged ions in nature, but no twice negatively charged ions. So it might even be that $N_{c}(Z)$ is always bounded by $Z+1$. In any event, there is a conjecture 580 that $N_{c}(Z) \leq Z+k$ for some finite $k$. It is known 413 that for fermion electrons, one has that $\lim _{Z \rightarrow \infty} N_{c}(Z) / Z=1$ but Benguria-Lieb [49] have proven that the liminf is strictly bigger than 1 for bosonic electrons. There is considerable literature since these two basic papers, but since this is already removed from Kato's work, we will not try to summarize it. 


\section{Eigenvalues, II: Lack of Embedded Eigenvalues}

Consider on $\mathbb{R}^{\nu}$, the equation $(-\Delta+V) \varphi=\lambda \varphi$ with $V(x) \rightarrow 0$ as $|x| \rightarrow \infty$ and $\lambda>0$. Naively, one might expect that no solution, $\varphi$, can be in $L^{2}\left(\mathbb{R}^{\nu}, d^{\nu} x\right)$. The intuition is clear: classically, if the particle is in the region $\{x|| x \mid>R\}$, where $R$ is picked so large that $|x|>R \Rightarrow V(x)<\lambda / 2$ and if the velocity is pointing outwards, the particle is not captured and so not bound. Due to tunneling, in quantum theory, a particle will always reach this region so there should not be positive energy bound states. This intuition of no embedded eigenvalues is incomplete due to the fact that bumps can cause reflections even when the bumps are smaller than the energy, so an infinite number of small bumps which do not decay too rapidly might be able to trap a particle. Indeed, in 1929, near the birth of modern quantum theory, von Neumann-Wigner 632 presented an example with an embedded eigenvalue of energy 1 (in fact they picked $V(x) \rightarrow-1$ at infinity and $\lambda=0$; we will shift energies by 1 and also pick their arbitrary constant $A$ to be 1$)$. They had the idea of guessing the wave function, $\psi$, and setting $V(x)=1+\psi^{-1} \Delta \psi(x)$. They picked $\psi$ so that it had oscillations that cancelled the +1 at infinity. Their choice as a function of $r=|x|$ in three dimensions was

$$
\psi(x)=\frac{\sin r}{r}\left[1+g(r)^{2}\right]^{-1} ; \quad g(r)=2 r-2 \sin (2 r)
$$

and they claimed that (where $\tilde{g}(r)=2 r+2 \sin (2 r)$ )

$$
V(x)=-32 \cos ^{4} r \frac{1-3 \tilde{g}(r)^{2}}{\left[1+\tilde{g}(r)^{2}\right]^{2}} .
$$

With slow enough decay, one can have much more than a single embedded eigenvalue. It is known (see [570, 373]) that if $0<\beta<1 / 2$ and $q_{\omega}(x)$ is a random potential in one dimension with uniformly spaced independent, identically distributed random bumps, then $-\frac{d^{2}}{d x^{2}}+\left(1+x^{2}\right)^{-\beta / 2} q_{\omega}(x)$ has only dense pure point spectrum, i.e. the essential spectrum is $[0, \infty)$ and there is a complete orthonormal set of $L^{2}$ eigenvectors!

In 1959, Kato proved the first strong result on the non-existence of positive eigenvalues.

Theorem 12.1 ([317], announced in [316]). Let $V(x)$ be continuous on $\mathbb{R}^{\nu}$ and obey

$$
\lim _{r \rightarrow \infty} r \sup _{|y|>r}|V(y)|=0 .
$$

Then $(-\Delta+V) \varphi=\lambda \varphi$ with $\lambda>0$ has no (nonzero) $L^{2}$ solutions.

Remarks . (1) ODE techniques easily prove in one dimension and in arbitrary dimension if $V$ is spherically symmetric, that there are no positive eigenvalues if $\int_{1}^{\infty}|V(r)| d r<\infty$. This goes back at least to Weyl 644 who quotes the results of Kneser 363. In modern parlance, it follows from the existence of Jost solutions. 
(2) Earlier, Brownell [71, Theorem 6.7] proved the absence of such eigenvalues under bounds of the form $|V(x)| \leq C_{1} \exp \left(-C_{2}|x|\right)$.

(3) There is both earlier and illuminating later work in the one-dimensional (equivalently spherical symmetric) case. Let

$$
K \equiv \limsup _{|x| \rightarrow \infty}[|x||V(x)|] .
$$

Kato proved in general dimension that there are no eigenvalues, $E$, with $E \geq K^{2}$. The (corrected) Wigner-von Neumann example has $K=8, E=1$ so one knows from that one cannot do better than $K^{2} / 64$ and it is easy to modify this example to show that one cannot do better than $K^{2} / 4$. In 1948, Wallach [634] proved the $E \leq K^{2}$ in one dimension (extended by Borg [63] and Eastham [126]) and provided an example showing that one could not do better than $K^{2} / 4$. A breakthrough in this one-dimensional case was made by Atkinson-Everitt [22] who proved that there is no eigenvalue if $E \geq 4 K^{2} / \pi^{2}$ and that there are examples with eigenvalues arbitrarily close to this bound. Note that $4 / \pi^{2}=0.405 \ldots$ lies in $(1 / 4,1)$. Their example is a relative of the Wigner-von Neumann example but uses $\operatorname{sgn}(\sin (r))$ in place of $\sin (r)$. Their method using Prüfer transforms is much of one-dimensional. Eastham-Kalf 128 give a textbook presentation of this work and mention that Halvorsen (unpublished) also found the optimal $4 K^{2} / \pi^{2}$. Remling 489] extended the AtkinsonEveritt result to prove no singular continuous spectrum in $\left[4 K^{2} / \pi^{2}, \infty\right)$.

(4) Kato proved results about more than $L^{2}$ solutions. For example, he proved that if $|V(x)| \leq(1+|x|)^{-\alpha}$ near infinity with $\alpha>1$, and if $(-\Delta+V) \varphi=\lambda \varphi$ with $\lambda>0$ with $\varphi(x) \rightarrow 0$ as $x \rightarrow \infty$, then $\varphi$ vanishes near infinity (and depending on the structure of the singularities of $V$, one can often use unique continuation (see below) to conclude that $\varphi \equiv 0$ ). This will be useful in Sec. 15

The observant reader may have noted that since $g(r) / r \rightarrow 1$ as $r \rightarrow \infty$, the potential, $V(x)$, given by (12.2) is $\mathrm{O}\left(r^{-2}\right)$ so it seems to be a counterexample to Theorem 12.1 In fact, von Neumann-Wigner had a calculational error: in the middle, they used $\cos r / \sin r=\tan r(!)$ and this error produces a remarkable cancellation. Doing the calculation correctly yields

$$
V(r)=-32 \sin r \frac{g(r)^{3} \cos r-3 g(r)^{2} \sin ^{3} r+g(r) \cos r+\sin ^{3} r}{\left[1+g(r)^{2}\right]^{2}}
$$

so that $V(r)=-8 \sin (2 r) / r+\mathrm{O}\left(r^{-2}\right)$ consistent with Kato's theorem. I once pointed out this error to Wigner, who thought for a moment and then said to me: "Oh, Johnny did that calculation".

Kato proved some differential inequalities on $M(r)=r^{\nu-1} \int|\varphi(r \omega)|^{2} d \omega$ (where $d \omega$ is surface measure on the unit sphere) and used them to prove that if $\int^{\infty} M(r) d r<\infty$ (i.e. $\varphi \in L^{2}\left(\mathbb{R}^{\nu}\right)$ ), then $M(r)=0$ for $r>R_{0}$ for some $R_{0}$. The final step in his proof needs a result that any solution of $(-\Delta+W) \varphi=0$ that vanishes on an open set is identically zero. This is called a unique continuation 
theorem (we note the analog fails for hyperbolic equations). Such theorems go back to Carleman [80] in 1939. He only treated $\nu=2$ and required that $V \in L^{\infty}$. The kind of estimates he used, now called Carleman estimates, have been a staple, not only of later work on unique continuation, but for many other topics in the theory of elliptic PDEs. Unique continuation when $V \in L^{\infty}$ and $\nu \geq 3$ was proven by Müller [432] in 1954 (see also [17]). So when Kato did his work, there was only unique continuation for bounded $V^{\prime} s$. Thus, in the final step, one needs to know there is a compact set, $S$, of measure zero so that $\mathbb{R}^{\nu} \backslash S$ is connected and so that $V$ is locally bounded on this connected set.

Starting in 1980, there were a number of unique continuation results with $L_{\text {loc }}^{p}$ conditions on $V$ culminating in the classic 1985 paper of Jerison-Kenig [278] who require (for $\nu \geq 3$; for $\nu=2$, the condition is more complicated) that $V \in L_{\mathrm{loc}}^{\nu / 2}$ which is known to be optimal.

In fact, one only needs something weaker than unique continuation, namely that there are no eigenfunctions of compact support. We will discuss this shortly.

Ikebe-Uchiyama [256] extended Kato's result to allow magnetic fields which are $\mathrm{o}\left(x^{-1}\right)$ at infinity and Roze [505] allowed suitable non-constant coefficient secondorder elliptic term.

Froese et al. 172 proved a variant of Kato's result. They first proved that if $V$ is $-\Delta$-bounded and $(-\Delta+1)^{-1 / 2}(|x| V)(-\Delta+1)^{-1}$ is a compact operator, and if $(-\Delta+V) \varphi=\lambda \varphi, \varphi \in D(H)$ and $\lambda>0$, then $e^{\alpha|x|} \varphi \in L^{2}$ for all $\alpha>0$. They then prove (and this also shows no compact support eigenfunctions) that if $V(-\Delta+1)^{-3 / 4}$ is bounded, $\lim _{\gamma \rightarrow \infty},\left\|V(-\Delta+\gamma)^{-3 / 4}\right\|=0$ and $\lim _{R \rightarrow \infty}\left\|\chi_{R}(1+|x|) V(-\Delta+1)^{-3 / 4}\right\|=0$ (where $\chi_{R}$ is the characteristic function of $\{x|| x \mid>R\})$, then $(-\Delta+V) \varphi=\lambda \varphi$ and $e^{\alpha|x|} \varphi \in L^{2}$ for all $\alpha>0 \Rightarrow \varphi=0$. This provides a proof of a variant of Kato's theorem without a need for pointwise bounds on $V$.

A very interesting alternate proof to a theorem very close to Kato is due to Vakulenko 626]. While Vakulenko and Yafaev 668] (who have a clear exposition of Vakulenko's work) say that he recovers Kato's result, instead he has a condition for a class of $V$ 's with lots of overlap to, but distinct from, Kato's condition (12.3). A Vakulenko bounding function, $\eta(r)$, is a function on $(0, \infty)$ obeying

$$
\forall_{r \in(0, \infty)} \eta(r)>0 ; \quad \lim _{r \downarrow 0} r \eta(r)=0 ; \quad \int_{0}^{\infty} \eta(r) d r<\infty .
$$

A Vakulenko potential, $V(x)$, on $\mathbb{R}^{\nu}$ is a measurable function for which there exists a Vakulenko bounding function, $\eta(r)$ with

$$
|V(x)| \leq \eta(|x|)
$$

If $\eta(x)=(1+|x|)^{-1-\epsilon}$ and $V$ obeys (12.7), then $V$ obeys both Vakulenko's condition and Kato's (12.3). If we consider $V(x)=(1+|x|)^{-1}[\log (2+|x|)]^{-\alpha}$, then $V$ obeys (12.3) if $\alpha>0$ but is only a Vakulenko potential if $\alpha>1$. On the other 
hand, if

$$
V(x)= \begin{cases}|x|^{-\beta} & \text { if for some } n=1,2, \ldots, n^{2}<|x|<n^{2}+1, \\ 0 & \text { otherwise, }\end{cases}
$$

then $V(x)$ obeys Kato's (12.3) only if $\beta>1$ but is a Vakulenko potential if $\beta>1 / 2$. So neither class is contained in the other, although they are very close. There is, of course, a connection to his condition and the fact that in one dimension, it has been long known that if the potential is in $L^{1}$, then the positive spectrum is purely absolutely continuous (as mentioned in Remark 1 after Theorem 12.1).

Theorem 12.2 ([626]). Let $V(x)$ be a Vakulenko potential with (12.7) for some $\eta$. Let $H=-\Delta+V$ and let $B$ be multiplication by $\sqrt{\eta}$. Then for any $0<a<$ $b<\infty$, there is a relatively $H$-bounded operator, $A$, so that for all $\lambda \in[a, b]$ and all $\varphi \in D(H)$, we have that

$$
\operatorname{Re}\langle(H-\lambda) \varphi, A \varphi\rangle \geq\|B \varphi\|^{2}
$$

In Sec.15, we will see that (12.9) has implications for local smoothness of $B$ and implies strong spectral properties of $H$. We will also prove the theorem when $\nu=1$ and say something about the proof for general $\nu$. For now, we note the following.

Corollary 12.3 ([626]). If $V$ is a Vakulenko potential and $H=-\Delta+V$, then $H$ has no positive eigenvalues.

Proof. Let $\lambda>0$. Pick $a, b$ with $0<a<\lambda<b<\infty$. If $H \varphi=\lambda \varphi$ for $\varphi \in D(H)$, by (12.9), we have that $\|B \varphi\|=0$. Since $\eta$ is everywhere non-vanishing, we conclude that $\varphi=0$.

The Wigner-von Neumann example has oscillations and one expects that if such oscillations are absent, then there should also be no positive eigenvalues. For example, if $V(x)$ looks like $r^{-\alpha}, 0<\alpha \leq 1$, one expects that there should also be no positive eigenvalues. Odeh 451 ] proved that if $\boldsymbol{x} \cdot \boldsymbol{\nabla} V \leq 0$ for all large $x$, then Kato's method could be modified to show that there are no positive eigenvalues. Shortly thereafter, Agmon [2] and Simon [540], using Kato's methods, independently proved (with enough local regularity to apply a unique continuation theorem) that there are no positive eigenvalues if $V(x)=V_{1}(x)+V_{2}(x)$ so long as when $x \rightarrow \infty$, one has that $|x|\left|V_{1}(x)\right| \rightarrow 0, V_{2}(x) \rightarrow 0$ and $\boldsymbol{x} \cdot \nabla V_{2}(x) \rightarrow 0$. Most later works and, in particular, both Froese et al. [172] and Vakulenko 626], also considered such sums. Khosrovshahi-Levine-Payne 354] and Kalf-Krishna Kumar [288] allow a third highly oscillatory piece and prove no positive eigenvalues (so for example, they allow $r^{-1} \sin \left(r^{\beta}\right)$ for $\beta>1$ and Agmon-Simon allow $\beta<1$ ).

Another way of extending Odeh's result proves the absence of positive eigenvalues using the virial theorem as discussed as follows (see also the discussion of Lavine's work in Sec. 15).

Before discussing more results on the absence of positive energy eigenvalues, we pause for some other examples, motivated by the Wigner-von Neumann example, 
where there are positive energy eigenvalues. By taking suitable sums of $b_{j} \sin \left(\alpha_{j} r\right) / r$ (cutoff away from infinity), Naboko [434] and Simon [579] constructed, for each $\delta>0, V(x)$, bounded by $r^{-1+\delta}$ near infinity with dense point spectrum. In what follows is one such result (taken from 579 ).

Theorem 12.4. For any countable subset $\left\{E_{k}\right\}_{k=1}^{\infty}$ of $(0, \infty)$ and any $\epsilon, \delta>0$, there is $V(x)$ on $(0, \infty)$ so that $-\frac{d^{2}}{d x^{2}}+V(x)$ on $L^{2}(0, \infty ; d x)$ with $\varphi(0)=0$ boundary conditions has $\varphi_{k} \in L^{2} \cap C^{2}(0, \infty)$, so $\varphi_{k}(0)=0$ and $-\varphi_{k}^{\prime \prime}+V \varphi_{k}=E_{k} \varphi_{k}$ and so that

$$
|V(x)| \leq \epsilon(1+|x|)^{-1+\delta} .
$$

Remark. If $0<\delta<1 / 2$, it is known [90, 489, 105, 356] that $-\frac{d^{2}}{d x^{2}}+V(x)$ has a.c. spectrum on all of $[0, \infty)$ so this is point spectrum embedded in continuous spectrum. As noted already, if $\delta>1 / 2$, one can find $V$ 's with only point spectrum.

The Wigner-von Neumann and Naboko-Simon examples are spherically symmetric. Ionescu-Jerison [257] found examples where the slow $\mathrm{O}\left(r^{-1}\right)$ decay is only in a parabolic tube about a single direction.

Theorem 12.5 ([257]). Fix $\nu \geq 2$. There exists $C>0$ and for each $n=1,2, \ldots$, a potential obeying

$$
\left|V\left(x_{1}, \ldots, x_{\nu}\right)\right| \leq \frac{C}{n+\left|x_{1}\right|+\left|x_{2}\right|^{2}+\cdots+\left|x_{\nu}\right|^{2}}
$$

and so that $(-\Delta+V) \varphi=\varphi$ has a nonzero $L^{2}$ solution.

Frank-Simon [160] have simplified the Ionescu-Jerison construction by hewing more closely to the Wigner-von Neumann method. They use the wave function

$$
\varphi_{n}(x)=\sin x_{1}\left(n^{2}+g\left(x_{1}\right)^{2}+\left(x_{2}^{2}+\cdots+x_{\nu}^{2}\right)^{2}\right)^{-\alpha},
$$

where $\alpha>\nu / 4$ (which implies that $\psi_{n} \in L^{2}$ ) and $g$ is given by (12.1). $V_{n}$ is then defined by

$$
V_{n}(x)=\frac{\Delta \psi_{n}+\psi_{n}}{\psi_{n}}
$$

which is seen to obey (12.11). 160 also has versions of the central Wigner-von Neumann potentials for dimensions different from 1 and 3.

Note that (12.11) implies that $V_{n} \in L^{p}\left(\mathbb{R}^{\nu}\right)$ for any $p>\frac{1}{2}(\nu+1)$. That says that the value of $p$ in the following is optimal.

Theorem 12.6 $([370])$. Let $\nu \geq 2$. If $V \in L^{p_{1}}\left(\mathbb{R}^{\nu}\right)+L^{p_{2}}\left(\mathbb{R}^{\nu}\right)$, where $p_{1}=\frac{1}{2} \nu<$ $p_{2}=\frac{1}{2}(\nu+1)\left(\right.$ if $\nu=2$, one needs to take $\left.p_{1}>1\right)$, then $-\Delta+V$ has no eigenvalues in $(0, \infty)$.

Remarks. (1) Earlier Ionescu-Jerison [257] proved the weaker result where $p_{2}=$ $\frac{1}{2}(\nu+1)$ is replaced by $p_{2}=\frac{1}{2} \nu$. 
(2) As we noted above, by Theorem 12.5, $p_{2}=\frac{1}{2}(\nu+1)$ is optimal. The lower bound on $p$ is needed to assure esa- $\nu$.

(3) The proof relies on $L^{p}$ Carleman estimates and the machinery of 369].

In many ways, the subtlest results on the absence of positive eigenvalues concern $N$-body systems. After all, we saw in Secs. 3 and 4 in Part 1 (Example 3.2 and Example 3.2 revisited) that $N$-body systems can have eigenvalues embedded in negative continua without carefully tuned potentials due to either non-interacting clusters or due to an eigenvalue of one symmetry embedded in a continuum of another symmetry. The earliest $N$-body results involve the Virial Theorem and showed no positive eigenvalues under specialized circumstances, for example, repulsive potentials and also $V^{\prime}$ 's homogeneous of degree $\beta$ (i.e. $V(\lambda \vec{x})=\lambda^{\beta} V(\vec{x}), 0>$ $\beta>-2$ ) which includes the physically important Coulomb case. This is discussed in 639, 8, 285. (or 479, Theorems XIII.59 and XIII.60]).

Undoubtedly, the deepest results on lack of positive eigenvalues for $N$-body systems are in Froese-Herbst [170]. They assume that the $V_{i j}(r)=v_{i j}\left(r_{i}-r_{j}\right)$, where $v_{i j}$ as functions on $\mathbb{R}^{\nu}$ obey $v_{i j}(-\Delta+1)^{-1}$ and $(-\Delta+1)^{-1}\left(y \cdot \nabla_{y} v_{i j}\right)(y)$ $(-\Delta+1)^{-1}$ are compact (here $\Delta$ is the Laplacian and all operators act on $\left.\mathbb{R}^{\nu}\right)$. These hypotheses are made so that Mourre theory applies (see [431, 465, 171, 12, 507]).

One takes $N$ particles $\left(x_{1}, \ldots, x_{N}\right), x_{j} \in \mathbb{R}^{\nu}$ and defines

$$
|x|=\left(2 \sum_{j=1}^{N} m_{j}\left|x_{j}-R\right|^{2}\right)^{1 / 2}
$$

where $R=\left(\sum_{j=1}^{N} m_{j}\right)^{-1}\left(\sum_{j=1}^{N} m_{j} x_{j}\right)$. If we are looking at a Hamiltonian on $L^{2}\left(\mathbb{R}^{\nu(N-1)}\right)$ with center of mass motion removed or if we have some $v_{j}$ representing interactions with infinite mass particles, then we act on $L^{2}\left(\mathbb{R}^{\nu N}\right)$, and set $R=0$. What Froese-Herbst found is the following.

Theorem 12.7 ([170]). Under the above hypotheses, if $H \psi=\lambda \psi, \psi \in L^{2}\left(\mathbb{R}^{\kappa}\right)$, then

$$
\beta \equiv \sup _{\alpha \geq 0}\left\{\alpha^{2}+\lambda \mid e^{\alpha|x|} \psi \in L^{2}\right\} \in \mathcal{T} \cup\{\infty\}
$$

where $\mathcal{T}$ is the set of thresholds of the system (see Sec. 11 for a discussion of thresholds).

If there are no positive thresholds (which one can prove inductively if there is a way to prove no positive eigenvalues), then if $\lambda>0$, the $\beta$ in (12.15) must be $\infty$. For suitable two-body systems, we saw above that eigenfunctions cannot obey $e^{\alpha|x|} \psi \in L^{2}$ for all $\alpha>0$. Froese et al. [173] proved the same for suitable $N$-body 
systems (see the paper for precise conditions); see also [571, Theorem C.3.8]. In this way, one proves that certain $N$-body systems have no positive eigenvalues.

The above touched on $L^{2}$ isotropic exponential bounds (and as we will see in Sec.19 that implies pointwise exponential bounds). There is a huge and beautiful literature on this subject and on non-isotropic bounds. We refer the reader to the book of Agmon [5] and the review article of Simon [571] which contains many references.

\section{Scattering and Spectral Theory, I: Trace Class Perturbations}

This is the first of four sections on spectral and scattering theory. For the 15 years between 1957 and 1972, this area was a major focus of Kato. When Kato was invited to give a plenary lecture at the 1970 International Congress of Mathematicians, his talk 326] was entitled "Scattering Theory and Perturbation of Continuous Spectra" (interestingly enough, Agmon and Kuroda gave invited talks at the same congress and spoke on closely related subjects). This section and the next two have brief introductory remarks introducing this subject. This section's introduction has much of the background we will give on scattering theory, the next section discusses the basics of spectral theory and something about the connection between timeindependent and time-dependent scattering theory and Sec. 15 will say more about the background behind the time-independent approach.

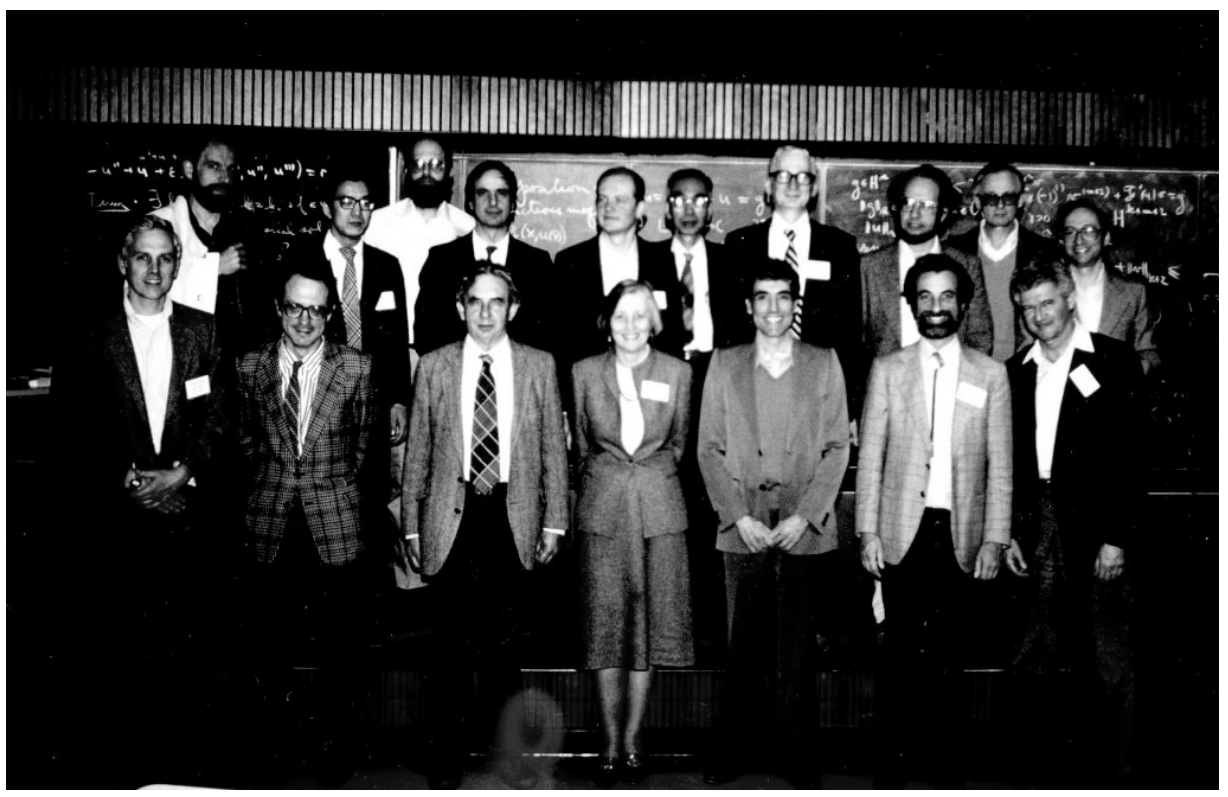

Birmingham, AL, Meeting on Differential Equations, 1983.

Back row: Fröhlich, Yajima, Simon, Temam, Enss, Kato, Schechter, Brezis, Carroll, Rabinowitz.

Front row: Crandall, Ekeland, Agmon, Morawetz, Smoller, Lieb, Lax. 


\section{B. Simon}

Starting with Rutherford's 1911 discovery of the atomic nucleus, scattering has been a central tool in fundamental physics, so it is not surprising that one of the first papers in the new quantum theory was by Born 64 on scattering. At its root, scattering is a time-dependent phenomenon: something comes in, interacts and moves off. But since it relied on eigenfunctions, Born's work used timeindependent objects. He assumed that one could construct non- $L^{2}$ eigenfunctions, $(-\Delta+V) \varphi=k^{2} \varphi,\left(\vec{k} \in \mathbb{R}^{3}, k=|\vec{k}|\right)$ which as $r \rightarrow \infty$ looks like

$$
\varphi(\vec{x}) \sim e^{i \vec{k} \cdot \vec{x}}+f(\theta) \frac{e^{i k r}}{r} ; \quad r=|\vec{x}|, \quad \vec{k} \cdot \vec{x}=k r \cos (\theta) .
$$

The time dependence gives $e^{-i t H} \varphi(x)$ a $e^{i \vec{k} \cdot(\vec{x}-\vec{k} t)}$ term which is a usual plane wave with velocity $\vec{k}$ and a scattered wave $f(\theta) r^{-1} e^{i k(r-k t)}$. One expects such a term to live near points, where $r=k t$. So if $t<0$, that term should not contribute (since $r>0$ ) while for $t$ positive and large, we have an outgoing spherical wave representing the scattering. We will say a little more about making mathematical sense of this formal argument in Sec. 15. $|f(\theta)|^{2}$ was then interpreted as a scattering differential cross-section. Born also found a leading-order perturbation formula for $f(\theta)$ :

$$
f(\theta)=-(2 \pi) \int e^{i\left(\overrightarrow{k^{\prime}}-\vec{k}\right) \cdot \vec{x}} V(\vec{x}) d \vec{x},
$$

where $k^{\prime}=k$ and $\overrightarrow{k^{\prime}} \cdot \vec{k}=k^{2} \cos \theta$. This Born approximation turns out to be leading order not only in $V$, but also for $V$ fixed as $k \rightarrow \infty$.

In the early 1940s, the theoretical physics community first considered timedependent approaches to scattering. Wheeler [646] and Heisenberg [219] defined the $S$-matrix and Møller [429] introduced wave operators as limits (with no precision as to what kind of limit).

It was Friedrichs in a prescient 1948 paper [166] who first considered the invariance of the absolutely continuous spectrum under sufficiently regular perturbations. Friedrichs was Rellich's slightly older contemporary. Both were students of Courant at Göttingen in the late 1920s (in 1925 and 1929, respectively). By 1948, Friedrichs was a professor at Courant's institute at NYU. Friedrichs considered two classes of examples in this paper. One was the model mentioned in Example 3.1 of a perturbation of an embedded point eigenvalue. The other was $H=H_{0}+\lambda K$, where $H_{0}$ is multiplication by $x$ on $L^{2}([0,1], d x)$ and $K$ is a Hermitian integral operator with an integral kernel $K(x, y)$ assumed to vanish on the boundary (i.e. if $x$ or $y$ is 0 or 1 ) and to be Hölder continuous in $x$ and $y$. Using what we would call time-independent methods, Friedrichs constructed unitary operators, $U_{\lambda}$, for $\lambda$ sufficiently small, so that

$$
H_{0}+\lambda K=U_{\lambda} H_{0} U_{\lambda}^{-1}
$$

While Friedrichs neither quoted Møller nor ever wrote down the explicit formulae

$$
\Omega^{ \pm}\left(H, H_{0}\right)=\mathrm{s}^{-} \lim _{t \rightarrow \mp \infty} e^{i t H} e^{-i t H_{0}}
$$


(we remind the reader that the strange \pm versus $\mp$ convention that we use is universal in the theoretical physics community and uncommon among mathematicians and is not the convention that Kato used), he did prove something equivalent to showing that the limit $\Omega^{+}$existed and was $U_{\lambda}$ and that the limit $\Omega^{-}$existed and was equal to $S_{\lambda} \Omega^{+}$. Here $S_{\lambda}$ was an operator he constructed and identified with the $S$-matrix (although it differs slightly with what is currently called the $S$-matrix).

Motivated in part by Friedrichs, in 1957, Kato published two papers [313, 314] that set out the basics of the theory we will discuss in this section. In the first, he had the important idea of defining

$$
\Omega^{ \pm}(A, B)=\mathrm{s}-\lim _{t \rightarrow \mp \infty} e^{i t A} e^{-i t B} P_{\mathrm{ac}}(B),
$$

where $P_{\text {ac }}(B)$ is the projection onto $\mathcal{H}_{\mathrm{ac}}(B)$, the set of all $\varphi \in \mathcal{H}$ for which the spectral measure of $B$ and $\varphi$ is absolutely continuous with respect to Lebesgue measure (see [587] Sec. 5.1] or the discussion at the start of Sec. [14). If these strong limits exist, we say that the wave operators $\Omega^{ \pm}(A, B)$ exist.

By replacing $t$ by $t+s$, one sees that if $\Omega^{ \pm}(A, B)$ exist, then $e^{i s A} \Omega^{ \pm}=$ $\Omega^{ \pm} e^{i s B}$. Since $\Omega^{ \pm}$are unitary maps, $U^{ \pm}$, of $\mathcal{H}_{\mathrm{ac}}(B)$ to their ranges, we see that $U^{ \pm} B \nmid \mathcal{H}_{\mathrm{ac}}(B)\left(U^{ \pm}\right)^{-1}=A\left\lceil\operatorname{ran} \Omega^{ \pm}\right.$. In particular, ran $\Omega^{ \pm}$are invariant subspaces for $A$ and lie in $\mathcal{H}_{\text {ac }}(A)$. It is thus natural to define: $\Omega^{ \pm}(A, B)$ are said to be complete if

$$
\operatorname{ran} \Omega^{+}(A, B)=\operatorname{ran} \Omega^{-}(A, B)=\mathcal{H}_{\mathrm{ac}}(A) .
$$

Remarks. (1) Kato also noted the relation

$$
\Omega^{ \pm}(A, B) \Omega^{ \pm}(B, C)=\Omega^{ \pm}(A, C)
$$

in that if both wave operators on the left exist, so does the one on the right and one has the equality.

(2) The wisdom of taking $P_{\mathrm{ac}}(B)$ in the definition of wave operator is shown by the fact that it follows from the results of Aronszajn [18] and Donoghue [120] (see also [564]) that if $A-B=\langle\varphi, \cdot\rangle \varphi$ with $\varphi$ a cyclic vector for $B$, then $e^{i t A} e^{-i t B} \psi$ has a limit if and only if $\psi \in \mathcal{H}_{\mathrm{ac}}(B)$.

In 313, Kato proved the following.

Theorem 13.1 ([313]). Let $\Omega^{ \pm}(A, B)$ exist. Then they are complete if and only if $\Omega^{ \pm}(B, A)$ exist.

The proof is almost trivial. It depends on noting that

$$
\psi=\lim _{t \rightarrow \infty} e^{i A t} e^{-i t B} \varphi \Leftrightarrow \varphi=\lim _{t \rightarrow \infty} e^{i t B} e^{-i t A} \psi
$$

since

$$
\left\|\psi-e^{i A t} e^{-i t B} \varphi\right\|=\left\|e^{i t B} e^{-i t A} \psi-\varphi\right\| .
$$

That said, it is a critical realization because it reduces a completeness result to an existence theorem. In particular, it implies that symmetric conditions which imply existence also imply completeness. We will say more about this as follows. 
To show the importance of this idea, motivated by it in [107, Deift and Simon proved that completeness of multichannel scattering for $N$-body scattering was equivalent to the existence (using the $N$-body language of Sec. 11) of $\mathrm{s}-\lim _{t \rightarrow \pm \infty} e^{i t H}(\mathcal{C}) J_{\mathcal{C}} e^{-i t H} P_{\text {ac }}(H)$ for the partition of unity $\left\{J_{\mathcal{C}}\right\}_{\mathcal{C} \neq \mathcal{C}_{\text {min }}}$ discussed in Remark 4 after Theorem 11.8 All proofs of asymptotic completeness for $N$-body systems prove it by showing the existence of these Deift-Simon wave operators in support of Kato's Theorem 13.1.

In 313, Kato proved the following.

Theorem 13.2 ([313]). Let $H_{0}$ be a self-adjoint operator and $V$ a (bounded) selfadjoint, finite rank operator. Then $H=H_{0}+V$ is a self-adjoint operator and the wave operators $\Omega^{ \pm}\left(H, H_{0}\right)$ exist and are complete.

This implies the unitary equivalence of $H_{0} \uparrow \mathcal{H}_{\mathrm{ac}}\left(H_{0}\right)$ and $H \uparrow \mathcal{H}_{\mathrm{ac}}(H)$. Remarkably, in the same year, Aronszajn [18] proved that this invariance holds for finite rank perturbations of boundary conditions for Sturm-Liouville operators (extended later using similar ideas by Donoghue [120] to general finite rank perturbations). Their methods are totally different from Kato's and do not involve wave operators.

Later in 1957, Kato [314] proved the following.

Theorem 13.3 (Kato-Rosenblum Theorem). The conclusions of Theorem 13.2 remain true if $V$ is a (bounded) trace class operator.

In a sense this theorem is optimal. It is a result of Weyl-von Neumann 643, 631] (see [587, Theorem 5.9.2]) that if $A$ is a self-adjoint operator, one can find a HilbertSchmidt operator, $C$, so that $B=A+C$ has only pure point spectrum. Kato's student, Kuroda [380], shortly after Kato proved Theorem 13.3, extended this result of Weyl-von Neumann to any trace ideal strictly bigger than trace class. So within trace ideal perturbations, one cannot do better than Theorem 13.3 .

The name given to this theorem comes from the fact that before Kato proved Theorem 13.3 Rosenblum 503 proved a special case that motivated Kato: namely, if $A$ and $B$ have purely a.c. spectrum and $A-B$ is trace class, then $\Omega^{ \pm}(A, B)$ exist and are unitary (so complete).

I had always assumed that Rosenblum's paper was a rapid reaction to Kato's finite rank paper which, in turn, motivated Kato's trace class paper. But I recently learned that this assumption is not correct. Rosenblum was a graduate student of Wolf at Berkeley who submitted his thesis in March 1955. It contained his trace class result with some additional technical hypotheses; a December 1955 Berkeley technical report had the result as eventually published without the extra technical assumption. Rosenblum submitted a paper to the American Journal of Mathematics which took a long time refereeing it before rejecting it. In April 1956, Rosenblum submitted a revised paper to the Pacific Journal in which it eventually appeared (this version dropped the technical condition; I have no idea what the original journal submission had). 
Kato's finite rank paper was submitted to J. Math. Soc. Japan on March 15, 1957 and was published in the issue dated April, 1957(!). The full trace class result was submitted to Proc. Japan Acad. on May 15, 1957. Kato's first paper quotes an abstract of a talk Rosenblum gave to an Amer. Math. Soc. meeting but I do not think that abstract contained many details. This finite rank paper has a note added in proof thanking Rosenblum for sending the technical report to Kato, quoting its main result and saying that Kato had found the full trace class results ("Details will be published elsewhere."). That second paper used some technical ideas from Rosenblum's paper.

I have heard that Rosenblum always felt that he had not received sufficient credit for his trace class paper. There is some justice to this. The realization that trace class is the natural class is important. As I have discussed, trace class is maximal in a certain sense. Kato was at Berkeley in 1954 when Rosenblum was a student (albeit some time before his thesis was completed) and Kato was in contact with Wolf. However, there is no indication that Kato knew anything about Rosenblum's work until shortly before he wrote up his finite rank paper when he became aware of Rosenblum's abstract. My surmise is that both, motivated by Friedrichs, independently became interested in scattering.

It should be emphasized that 1956-1957 was a year that (time-dependent) scattering theory seemed to be in the air. Cook 93. found a simple, later often used, method for proving that $\Omega^{ \pm}(A, B)$ exists: if $\int_{-\infty}^{\infty}\left\|(A-B) e^{-i u B} \varphi\right\| d u<\infty$, then by integrating a derivative

$$
\limsup _{\substack{t, s \rightarrow \infty \\ \text { or } t, s \rightarrow-\infty}}\left\|e^{i t A} e^{-i t B} \varphi-e^{i s A} e^{-i s B} \varphi\right\| \leq \lim \int_{s}^{t}\left\|(A-B) e^{-i u B} \varphi\right\| d u=0
$$

so it suffices that

$$
\int_{-\infty}^{\infty}\left\|(A-B) e^{-i u B} \varphi\right\| d u<\infty
$$

for a dense set of $\varphi$ for $\Omega^{ \pm}(A, B)$ to exist. Cook applied this to $B=-\Delta ; A=$ $-\Delta+V ; V \in L^{2}\left(\mathbb{R}^{3}\right)$ (which translates to $\mathrm{O}\left(|x|^{-3 / 2-\epsilon}\right.$ ) decay). Hack [206] and Kuroda 381 extended this to allow $\mathrm{O}\left(|x|^{-1-\epsilon}\right)$ decay.

Since, for the free dynamics, $x \sim c t$, one expects and can prove that if $\alpha \leq 1$, then $\int_{-\infty}^{\infty}\left\|(1+|x|)^{-\alpha} e^{i u \Delta} \varphi\right\| d u=\infty$ for all $\varphi$. Indeed, Dollard [118] showed that one needs modified wave operators for Coulomb potentials (again, there is a large literature on the subject of Coulomb or slower decay of which we mention [90, 113]).

Extensions of Cook's ideas and other scattering theory notions to quadratic form perturbations can be found in [383, [511, 557] 337. Kato states his results in a two-Hilbert space setting (see as follows). $J$ is a bounded linear operator from $\mathcal{H}_{1}$ to $\mathcal{H}_{2}$ and $H_{j}$ are self-adjoint operators on $\mathcal{H}_{j} ; j=1,2$. For $z \in \mathbb{C} \backslash \mathbb{R}$, let $C(z)=\left(H_{2}-z\right)^{-1} J-J\left(H_{1}-z\right)^{-1}$. Kato proves that if for some $z$ and $\varphi \in \mathcal{H}_{1}$, one 


\section{B. Simon}

has that

$$
\int_{0}^{\infty}\left\|C(z) e^{-i t H_{1}} \varphi\right\|_{2} d t<\infty
$$

then

$$
\lim _{t \rightarrow \infty} e^{i t H_{2}} J e^{-i t H_{1}} \varphi \text { exists. }
$$

He then shows that this allows some cases where $H_{2}$ is only defined as a quadratic form, e.g. $H_{1}=-\Delta, H_{2}=-\Delta+V$ with $V \geq 0, V \in L^{1}\left(\mathbb{R}^{3},(1+|x|)^{1-\epsilon} d x\right)$.

For many years, it was thought that this simple idea of Cook was limited to existence but not useful for completeness or spectral theory. This was overturned by a brilliant paper of Enss [136] (see also [464, 478, Sec. XI.17] or [562]), a subject we will not pursue here.

In 1958-1959, there were also several influential papers by Jauch [269, 270] that discussed scattering in a general framework.

In considering extensions of the Kato-Rosenblum, I begin with four issues that involve work by Kato himself. First, we discuss proofs. Like Friedrichs, both Kato and Rosenblum proved that a time-dependent limit exists by first constructing objects with time-independent methods which they prove is the required limit. The first fully time-dependent proof of Theorem 13.3 is in Japanese language paper by Kato 315. also published in 1957. His argument was repeated with permission in a paper by his student Kuroda [382. The slickest version of this time-dependent proof is in Kato's 1966 book [332. It is a variant of this argument that Pearson used in his proof of Theorem 13.4

The second concerns Kato's paper 321] on what is called the invariance principle: for suitable functions $\Phi$, one shows that $A-B$ trace class $\Rightarrow \Omega^{ \pm}(\Phi(A), \Phi(B))$ exist and are complete. In case that $\Phi$ is strictly monotone increasing (respectively, decreasing), one has that $\Omega^{ \pm}(\Phi(A), \Phi(B))=\Omega^{ \pm}(A, B)$ (respectively, $\Omega^{\mp}(A, B)$ ). The first examples of this phenomenon are due to Birman [54, 55]. Kato focused on the general form of the principle. There is a considerable literature on non-trace class versions of an invariance principle; see [478, Notes to Sec. XI.3] for references.

The third involves two-Hilbert space scattering theory 323. This came out of a set of concrete problems. In Sec. 8 in Part 1 (see the discussion beginning with (8.6)), we saw that the equation $\frac{\partial^{2} u}{\partial t^{2}}=(\Delta-V) u$ had a unitary propagation in the norm $\left[\|\dot{u}\|_{2}^{2}+\langle u,(-\Delta+V) u\rangle\right]^{1 / 2}$. This means to compare solutions of this equation to, say, the one with $V=0$, one needs to consider two different Hilbert space norms. If for some $0<\alpha<\beta<\infty$, one has for all $x$ that $\alpha \leq V(x) \leq \beta$, then there is a natural map, $J$ between the two spaces so that $J$ is bounded with bounded inverse which takes $\varphi$ viewed as an element of one Hilbert space into itself but viewed in other Hilbert space. One is interested in the limit in (13.13) (and also the limit as $t \rightarrow-\infty)$. A similar setup applies to other hyperbolic systems, especially to the physically significant Maxwell's equation. Long after Kato's work on the subject, Isozaki-Kitada 261] discovered that one could use a $J$ operator to discuss 
long-range scattering where ordinary wave operators do not exist. Before 323, several authors [512, 525, 614, 649] discussed scattering theory for some concrete examples of such systems. Kato [323] looked at the theory systematically, focusing, for example, on $J$ 's with $s$ - $\lim _{t \rightarrow \pm \infty}\left(J^{*} J-\mathbf{1}\right) e^{-i t H_{1}}=0$ which implies that the wave operators are isometries if they exist. Under certain invertibility hypotheses on $J$, Kato could carry over the usual trace class scattering theory to get some two Hilbert space results. Stronger results were subsequently obtained by Belopol'skiiBirman [43, Birman [57] and then Pearson [459] who proved the following.

Theorem 13.4 (Pearson's Theorem [459]). Let A, B be self-adjoint operators on Hilbert spaces $\mathcal{H}_{1}$ and $\mathcal{H}_{2}$. Let $J$ be a bounded operator from $\mathcal{H}_{1}$ to $\mathcal{H}_{2}$ so that $C=A J-J B$ is trace class (in the sense that there is a bounded operator $C$ from $\mathcal{H}_{1}$ to $\mathcal{H}_{2}$ with $\sqrt{C^{*} C}$ trace class and for $\varphi \in D(B)$ and $\psi \in D(A)$ we have that $\langle A \psi, J \varphi\rangle-\langle\psi, J B \varphi\rangle=\langle\psi, C \varphi\rangle)$. Then

$$
\Omega^{ \pm}(A, B ; J)=s-\lim _{t \rightarrow \mp \infty} e^{i t A} J e^{-i t B} P_{\mathrm{ac}}(B)
$$

exists.

No completeness is claimed (e.g. consider $J=0$ ) but one can sometimes get completeness. For example, if $\mathcal{H}_{1}=\mathcal{H}_{2}=\mathcal{H}$ and $A, B \geq 0$ are two positive operators on $\mathcal{H}$ so that $(A+1)^{-1}-(B+1)^{-1}$ is trace class, then one can pick $J=(A+$ $1)^{-1}(B+1)^{-1}$. $C$ is trace class, so $\Omega^{ \pm}(A, B ; J)$ exist. Apply this to $(B+1) \varphi$ to see that $\Omega^{ \pm}\left(A, B ;(A+1)^{-1}\right)$ exists. Since $(A+1)^{-1}-(B+1)^{-1}$ is compact, the Riemann-Lebesgue lemma shows that $\Omega^{ \pm}\left(A, B ;(A+1)^{-1}-(B+1)^{-1}\right)=0$. It follows that $\Omega^{ \pm}\left(A, B ;(B+1)^{-1}\right)$ exists. Applying this to $(B+1) \varphi$, we see that $\Omega^{ \pm}(A, B)$ exists. By symmetry, it is complete. We thus recover Birman's result (see as follows ) that $(A+1)^{-1}-(B+1)^{-1}$ trace class implies that $\Omega^{ \pm}(A, B)$ exists and is complete. Pearson's proof is a clever variant of Kato's time-dependent proof from [332]; see [478, pp. 33-38] for details and further applications.

Example 13.5. The fourth of Kato's applications/extensions of the trace class theory is an example in a joint paper with Kuroda 348. They consider three Hamiltonians on $L^{2}\left(\mathbb{R}^{2}, d^{2} x\right)$ :

$$
H_{0}=-\frac{\partial^{2}}{\partial x_{1}^{2}}-\frac{\partial^{2}}{\partial x_{2}^{2}} ; \quad H_{1}=H_{0}+V\left(x_{2}\right) ; \quad H=H_{1}+K,
$$

where $V \in L^{1}(\mathbb{R}) \cap L^{2}(\mathbb{R})$ and $K$ is a rank 1 operator, $K u=c\langle\varphi, u\rangle \varphi$ with $\varphi$ a norm 1 function in $L^{2}\left(\mathbb{R}^{2}\right)$ and $c$ is a constant. Moreover, they pick $V$ so that $h_{1}=-\frac{d^{2}}{d x^{2}}+V(x)$, as an operator on $L^{2}(\mathbb{R})$, has exactly one eigenvalue in $(-\infty, 0]$.

Let $h_{0}=-\frac{d^{2}}{d x^{2}}$. By results of Kuroda 381, using the trace class theory, $\Omega^{ \pm}\left(h_{1}, h_{0}\right)$ exist and are complete. Since $H_{0}, H_{1}$ are of the form $H_{j}=\mathbf{1} \otimes h_{j}+$ $h_{0} \otimes \mathbf{1}$, one sees that $\Omega^{ \pm}\left(H_{1}, H_{0}\right)$ exist with $\operatorname{ran} \Omega^{+}\left(H_{1}, H_{0}\right)=\operatorname{ran} \Omega^{-}\left(H_{1}, H_{0}\right)$. But they are not complete because $\mathcal{H}_{\mathrm{ac}}\left(H_{1}\right)$ has vectors of the form $\psi \otimes \varphi_{0}$, where $\psi \in L^{2}(\mathbb{R})$ and $\varphi_{0}$ is the bound state of $h_{1}$. 
Since $K$ is rank $1, \Omega^{ \pm}\left(H, H_{1}\right)$ exist and so by the chain rule $\Omega^{ \pm}\left(H, H_{0}\right)$ exist. But by a calculation, $K$ links the two parts of the a.c. spectrum of $H_{1}$, at least for $c$ small. Thus they claim that, for $c$ small, $\operatorname{ran} \Omega^{+}\left(H, H_{0}\right) \neq \operatorname{ran} \Omega^{-}\left(H, H_{0}\right)$ and the $S$-matrix is non-unitary. Hence the title of their paper "A Remark on the Unitarity Property of the Scattering Operator".

However, as Kuroda [379] subsequently noted, this analysis leaves something out. The $S$-matrix is unitary if one looks at the right $S$-matrix! This is a multichannel system and if one includes also the channel for $\left\{\psi \otimes \varphi_{0}\right\}$, the arguments do imply unitarity. So rather than finding a non-unitary $S$-matrix, they found the first example of a multichannel scattering system with asymptotic completeness!

We conclude this section with some brief remarks on developments in the trace class scattering theory subsequent to Kato's original work. Many of the significant results are due to Birman so much so that the theory has taken the name KatoBirman theory.

(1) A first key issue was making the theory apply to Schrödinger operators, $H_{0}=-\Delta, H=-\Delta+V$ on $L^{2}\left(\mathbb{R}^{\nu}\right)$. The pioneer was Kato's student, Kuroda, who first proved an extension of the Kato-Rosenblum theorem. If $V$ is $H_{0}$-bounded with relative bound less than 1 and $|V|^{1 / 2}\left(H_{0}+1\right)^{-1}$ is Hilbert-Schmidt, then Kuroda proved that $\Omega^{ \pm}\left(H, H_{0}\right)$ exist and are complete. He used this to prove existence and completeness if $\nu \leq 3$ and $V \in L^{1}\left(\mathbb{R}^{\nu}\right) \cap L^{2}\left(\mathbb{R}^{\nu}\right)$. In terms of $V^{\prime}$ s with

$$
|V(x)| \leq C(1+|x|)^{-\alpha},
$$

this requires $\alpha>\nu$ whereas the existence by Cook's method only needs $\alpha>1$, so for $\nu \geq 2$, there is a gap that we will discuss much more in the next two sections. Kuroda also noted that if $V(\vec{x})=V(|\vec{x}|)$ is a central potential, then, for any $\nu$, one can do a partial wave expansion (see [586. Theorem 3.5.8]) and reduce the problem to half-line problems. Since it is known that when (13.16) holds for any $\alpha>0$, that the essential spectrum for the half-line problem is $[0, \infty)$ and the spectrum is simple, one can see that the existence implies completeness without needing the trace class theory.

(2) Birman is responsible for a wide variety of extensions and applications of the trace class theory. First, he proved with Krein [58] an extension to the situation where $U$ and $V$ are two unitaries for which $V-U$ is trace class. In that case, $s-\lim _{n \rightarrow \pm \infty}\left(V^{*}\right)^{n} U^{n} P_{\mathrm{ac}}(U)$ exists, has range $\operatorname{ran} P_{\mathrm{ac}}(V)$ and is a unitary equivalence of the a.c. parts of $U$ and $V$. Second [54 55, he proved that if $A, B$ are self-adjoint and $(A-z)^{-1}-(B-z)^{-1}$ is trace class for some $z \notin \sigma(A) \cup \sigma(B)$, then $\Omega^{ \pm}(A, B)$ exist and are complete (deBranges [104 proved the same result). Kuroda's result on $|V|^{1 / 2}\left(H_{0}+1\right)^{-1}$ Hilbert Schmidt follows from this. Later Birman 56. proved that if $P_{I}(A)(A-B) P_{I}(B)$ is trace class for all bounded intervals, $I$, and if a technical condition called mutual subordinacy holds, then $\Omega^{ \pm}(A, B)$ exist and are complete. His proof was involved but using Pearson's Theorem (Theorem [13.4), one can easily prove this result of Birman (see [478, Theorem XI.10]). 
With this result, one can prove existence and completeness of $\Omega^{ \pm}\left(H, H_{0}\right)$ for $H_{0}=-\Delta, H=-\Delta+V$ on $L^{2}\left(\mathbb{R}^{\nu}\right)$ if $V \in L^{\nu / 2}\left(\mathbb{R}^{\nu}\right) \cap L^{1}\left(\mathbb{R}^{\nu}\right)$, so $\alpha>\nu$ in (13.16) leaving quite a gap from the expected $\alpha>1$ (see the next two sections).

(3) One can apply the trace class theory to the changes of boundary condition. The pioneer here is Birman [52 [53]; see also [106, Appendix].

(4) When $A$ and $B$ are bounded and $A-B$ is trace class, one can define an $L^{1}(\mathbb{R}, d x)$ function, $\xi(x)$, called the Krein spectral shift so that for $f$ a $C^{2}$ function of compact support, one has that $f(A)-f(B)$ is trace class and

$$
\operatorname{Tr}(f(A)-f(B))=-\int f^{\prime}(x) \xi(x) d x
$$

(see [564, Sec. 11.4] or [663, Chap. 8] for more on the spectral shift function). Birman-Krein [58] prove the beautiful Birman-Krein formula:

$$
\operatorname{det}(S(\lambda))=e^{-2 \pi i \xi(\lambda)}
$$

when $A-B$ is trace class. Here $S=\Omega^{-}(A, B)^{*} \Omega^{+}(A, B)$ is a unitary operator on $\mathcal{H}_{\text {ac }}(B)$ which commutes with $B$, so according to the spectral multiplicity theory [587. Sec. 5.4], $B$ has a direct integral decomposition $\mathcal{H}_{\mathrm{ac}}(B)=\int_{\sigma_{\mathrm{ac}}(B)}^{\oplus} \mathcal{H}_{\lambda} d \lambda, B=$ $\int_{\sigma_{\mathrm{ac}}(B)}^{\oplus}$ and $S=\int_{\sigma_{\mathrm{ac}}(B)}^{\oplus} S(\lambda) d \lambda$, where $S(\lambda)$ is a unitary operator on $\mathcal{H}_{\lambda}$. BirmanKrein prove that $S(\lambda)-\mathbf{1}$ is a trace class operator on $\mathcal{H}_{\lambda}$ and (13.18) holds where det is the Fredholm determinant [587 Sec. 3.10].

\section{Scattering and Spectral Theory, II: Kato Smoothness}

This is the second section on spectral and scattering theory. We begin with a quick primer on spectral theory that will assume familiarity with the spectral theorem and spectral measures (see [587, Secs. 5.1 and 7.2]). For a self-adjoint operator, $H$, on a (complex, separable) Hilbert space, $\mathcal{H}$, the most basic questions are connected to the Lebesgue decomposition theorem [583. Theorem 4.7.3] that says that any measure, $d \mu$ on $\mathbb{R}$ can be uniquely decomposed $d \mu=d \mu_{\mathrm{ac}}+d \mu_{\mathrm{sc}}+d \mu_{\mathrm{pp}}$, where $d \mu_{\mathrm{pp}}$ is pure point, $d \mu_{\mathrm{ac}}$ is $d x$-absolutely continuous and $d \mu_{\mathrm{sc}}$ has no pure points and is singular with respect to $d x$ (so "singular continuous"). There is a corresponding decomposition $\mathcal{H}=\mathcal{H}_{\mathrm{ac}}(H) \oplus \mathcal{H}_{\mathrm{sc}}(H) \oplus \mathcal{H}_{\mathrm{pp}}(H)$, where $\mathcal{H}_{y}$ is the set of those vectors, $\varphi$, whose $H$-spectral measure is purely of type $y$.

In simple quantum mechanical systems, $\mathcal{H}_{\text {ac }}$ spectrum is often associated with scattering theory as we have seen, and $\mathcal{H}_{\mathrm{pp}}$ is associated with bound states. As my advisor, Arthur Wightman, told me that there is no reasonable interpretation for states in $\mathcal{H}_{\mathrm{sc}}$, so he called the idea that $\mathcal{H}_{\mathrm{sc}}=\{0\}$ the "no goo hypothesis". A major concern of quantum theoretic spectral theorists in the period from 1960 to 1985 , and, in particular, of Kato, was the proof that $\mathcal{H}_{\mathrm{sc}}=\{0\}$ for two-(and $N$-)body quantum systems whose potentials obey (13.16) for $\alpha>1$.

Ironically, after Kato became less active in NRQM, it was discovered that, in some ways, singular continuous spectrum is ubiquitous. As I have remarked: "I seem 


\section{B. Simon}

to have spent the first part of my career proving that singular continuous spectrum never occurs and the second proving that it always does". A key breakthrough was the discovery by Pearson [460] that sparse potentials with slow decay have purely s.c. spectrum. I explored this in a series of papers [576, 110, 279, 109, 599, 577, 578] of which a typical result concerns $h$ on $\ell^{2}(\mathbb{Z})$ given by $(h u)_{n}=u_{n+1}+u_{n-1}+b_{n} u_{n}$. Fix $\alpha>0$ and let $Q_{\alpha}$ be the Banach space of $b^{\prime} s$ with $\sup _{n}\left[(1+|n|)^{\alpha}\left|b_{n}\right|\right] \equiv$ $\|b\|_{\alpha}<\infty$ with $|n|^{\alpha}\left|b_{n}\right| \rightarrow 0$ as $|n| \rightarrow \infty$. Then (see [576]), if $\alpha<1 / 2$, for a dense $G_{\delta}$ in $Q_{\alpha}$, the associated $h$ has purely s.c. spectrum (i.e. $\mathcal{H}_{\mathrm{sc}}(h)=\mathcal{H}$ ).

A main tool in the quest to prove that $\mathcal{H}_{\mathrm{sc}}=\{0\}$ is the fact that Stone's formula [587, Eq. (5.7.30)]

$$
\lim _{\epsilon \downarrow 0} \int_{a}^{b} \operatorname{Im}\langle\varphi, R(x+i \epsilon) \varphi\rangle d x=\left\langle\varphi, \frac{1}{2}\left[P_{(a, b)}(H)+P_{[a, b]}(H)\right] \varphi\right\rangle,
$$

(where $R(z)=(H-z)^{-1}$ for $\left.z \in \mathbb{C} \backslash \mathbb{R}\right)$ immediately implies that for any $p>1$, we have that

$$
\sup _{0<\epsilon<1} \int_{a}^{b}|\operatorname{Im}\langle\varphi, R(x+i \epsilon) \varphi\rangle|^{p} d x<\infty \Rightarrow P_{(a, b)}(H) \varphi \in \mathcal{H}_{\mathrm{ac}}(H) .
$$

Thus, the most common way of proving that $\mathcal{H}_{\text {sing }}=\{0\}$ is showing that for a dense set of $\varphi$, and enough intervals $(a, b)$, we have that

$$
\sup _{\substack{\epsilon>0 \\ a<x<b}}|\langle\varphi, R(x+i \epsilon) \varphi\rangle|<\infty
$$

(stronger than needed, but what one often gets).

We will say a lot more about time-independent scattering in the next section, but we note that in some sense, the key notion of that theory is that control of $\langle\varphi, R(x+i \epsilon) \varphi\rangle$ as $\epsilon \downarrow 0$ also says something about long time behavior of dynamics as seen in

$$
\int_{0}^{\infty} e^{-\epsilon t} e^{i t \lambda} e^{-i t H} \varphi d t=-i R(\lambda+i \epsilon) \varphi
$$

for any $\varphi \in \mathcal{H}$ because $\int_{0}^{\infty} e^{-\epsilon t} e^{i(\lambda-x) t} d t=-i(x-\lambda-i \epsilon)^{-1}$.

We turn now to the theory of Kato smoothness which is based primarily on two papers of Kato [322, 324]. The first is the basic one with four important results: the equivalence of many conditions giving the definition, the connection to spectral analysis, the implications for existence and completeness of wave operators and, finally, a perturbation result. The second paper concerns the Putnam-Kato theorem on positive commutators.

To me, the 1951 self-adjointness paper is Kato's most significant work (with the adiabatic theorem paper a close second), Kato's inequality his deepest and the subject of this section his most beautiful. One of the things that is so beautiful is that there is not just a relation between the time-independent and time-dependent objects - there is an equivalence! Here is the set of equivalent definitions. 
Theorem 14.1 ([322]). Let $H$ be a self-adjoint operator and $A$ a closed operator. The following are all equal $\left(R(\mu)=(H-\mu)^{-1}\right)$ :

$$
\begin{aligned}
& \sup _{\substack{\|\varphi\|=1 \\
\epsilon>0}} \frac{1}{4 \pi^{2}} \int_{-\infty}^{\infty}\left(\|A R(\lambda+i \epsilon) \varphi\|^{2}+\|A R(\lambda-i \epsilon) \varphi\|^{2}\right) d \lambda, \\
& \sup _{\|\varphi\|=1} \frac{1}{2 \pi} \int_{-\infty}^{\infty}\left\|A e^{-i t H} \varphi\right\|^{2} d t, \\
& \sup _{\substack{\|\varphi\|=1, \varphi \in D\left(A^{*}\right) \\
-\infty<a<b<\infty}} \frac{\left\|P_{(a, b)}(H) A^{*} \varphi\right\|^{2}}{b-a}, \\
& \sup _{\mu \notin \mathbb{R}, \varphi \in D\left(A^{*}\right)} \frac{1}{\|\varphi\|=1}\left|\left\langle A^{*} \varphi,[R(\mu)-R(\bar{\mu})] A^{*} \varphi\right\rangle\right|, \\
& \sup _{\mu \notin \mathbb{R}, \varphi \in D\left(A^{*}\right)} \frac{1}{\|\varphi\|=1} \\
& \left\|R(\mu) A^{*} \varphi\right\|^{2}|\operatorname{Im} \mu| .
\end{aligned}
$$

In particular, if one is finite (respectively, infinite), then all are.

Remarks. (1) In (14.4) / 14.5), we set $\|A \psi\|=\infty$ if $\psi \notin D(A)$, so, for example, to say that 14.5) is finite implies that for each $\varphi$, we have that $e^{-i t H} \varphi \in D(A)$ for Lebesgue a.e. $t \in \mathbb{R}$.

(2) If one and so all of the above quantities are finite we say that $A$ is $H$-smooth. The common value of these quantities is called $\|A\|_{H}^{2}$.

(3) The proof is not hard. If the integral in 14.5 has a factor of $e^{-2 \epsilon t}$ put inside it, the equality of the integrals in (14.4) and (14.5) follows from (14.3) and the Plancherel theorem. By monotone convergence, the sup of the time integral with the $e^{-2 \epsilon t}$ factor is the integral without that factor.

(4) The equivalence of (14.7) and (14.8) is just $R(\mu)-R(\bar{\mu})=(\mu-\bar{\mu}) R(\mu) R(\bar{\mu})$.

(5) If $d \nu_{A^{*} \varphi}$ is the $H$-spectral measure for $A^{*} \varphi$ (so $\int f(\lambda) d \nu_{A^{*} \varphi}(\lambda)=$ $\left\langle A^{*} \varphi, f(H) A^{*} \varphi\right\rangle$ ), then the equivalence of (14.6) and (14.7) involves the relation of $\frac{\epsilon}{\pi} \int \frac{d \nu(\lambda)}{(\lambda-x)^{2}+\epsilon^{2}}$ and $\frac{\nu((a, b))}{b-a}$. A bound like (14.6) implies a.e. in $d \lambda$ a bound on $\frac{d \nu(\lambda)}{d \lambda}$. Since $\frac{\epsilon}{\pi} \int \frac{d \lambda}{(\lambda-x)^{2}+\epsilon^{2}}=1$, we get (14.7). Conversely (14.7) implies (14.6) via Stone's formula.

(6) To see that 14.6 (14.4), it suffices by taking limits to consider the case where $a$ and $b$ are not eigenvalues of $H$. One writes $P_{(a, b)}(H)$ by Stone's formula to see that

$$
\begin{aligned}
\left|\left\langle A^{*} \varphi, P_{(a, b)}(H) \psi\right\rangle\right| & \leq \frac{1}{2 \pi}\|\varphi\| \limsup _{\epsilon \downarrow 0} \int_{a}^{b}\|A[R(\lambda+i \epsilon)-R(\lambda-i \epsilon)] \psi\| d \lambda \\
& \leq\|\varphi\|\left(\int_{a}^{b} 1 d \lambda\right)^{1 / 2}\left(\frac{1}{4 \pi^{2}} \int_{a}^{b} \text { Integrand in (14.4) } d \lambda\right)^{1 / 2}
\end{aligned}
$$

proving that $\left\|P_{(a, b)}(H) A^{*} \varphi\right\| \leq\|\varphi\|[\text { right-hand side of }(\underline{14.4})]^{1 / 2}|b-a|^{1 / 2}$. 
(7) To see that (14.4) $\leq 14.7$, thereby completing the proof of all the equivalences, let $\alpha$ be the sup in (14.7). For $z \in \mathbb{C}_{+}$, let $K(z)$ be the positive square root of $(2 \pi i)^{-1}(R(z)-R(\bar{z}))$. Then $\|A K(z)\|^{2} \leq \alpha$, so

Quantity whose sup is taken in (14.4) $=\int_{-\infty}^{\infty}\left\|A K(\lambda+i \epsilon)^{2} \varphi\right\|^{2} d \lambda$

$$
\leq \alpha \int_{-\infty}^{\infty}\|K(\lambda+i \epsilon) \varphi\|^{2} d \lambda=\alpha\|\varphi\|^{2} .
$$

(8) By (14.3), if $A$ is $H$-smooth, then

$$
\begin{aligned}
\|A R(\lambda+i \mu) \varphi\| & \leq \int_{0}^{\infty} e^{-\mu t}\left\|A e^{-i t H} \varphi\right\| d t \\
& \leq\left(\int_{0}^{\infty} e^{-2 \mu t} d t\right)^{1 / 2}\left(\int_{0}^{\infty}\left\|A e^{-i t H} \varphi\right\|^{2} d t\right)^{1 / 2} \\
& \leq(2 \mu)^{-1 / 2}(2 \pi)^{1 / 2}\|A\|_{H}
\end{aligned}
$$

so $A H$-smooth $\Rightarrow A$ is $H$-bounded with relative bound zero.

(9) In 322, Kato states this equivalence in stages since, as the title of the paper indicates, his focus is on controlling certain non-self-adjoint operators (we focus on the self-adjoint case of greatest interest in NRQM). He first considers general $H$ with $\sigma(H) \subset \mathbb{R}$ and proves a version of Theorem 14.6 and then (following Friedrichs [166]) constructs similarity operators using a stationary replacement for wave operators. He next adds to $H$ a condition that it generates a group $\{U(t)\}_{t \in \mathbb{R}}$ of bounded operators with $\|U(t)\|=\mathrm{O}\left(e^{\epsilon t}\right)$ for all $\epsilon>0$. Then (14.3) holds with $e^{-i t H}$ replaced by $U(t)$ and Kato proves the equality of (14.4) and (14.5) in that case. Finally, he proves the full Theorem 14.1 when $H$ is self-adjoint.

Example 14.2. Let $H=-i \frac{d}{d x}$ on $L^{2}(\mathbb{R})$ and let $A$ be multiplication by $f(x)$. Since $e^{-i t H} \varphi(x)=\varphi(x-t)$, we compute

$$
\int_{-\infty}^{\infty}\left\|A e^{-i t H} \varphi\right\|^{2} d t=\int_{\mathbb{R}^{2}} f(x)^{2} \varphi(x-t)^{2} d x d t=\|f\|_{2}^{2}\|\varphi\|_{2}^{2}
$$

so, if $f \in L^{2}(\mathbb{R})$, then $A$ is $H$-smooth.

Example 14.3. If $H_{0}$ is $-\Delta$ on $L^{2}\left(\mathbb{R}^{3}\right)$, it is known [583, $\left.(6.9 .48)\right]$ that $\left(H_{0}+\kappa^{2}\right)^{-1}$ with $\operatorname{Re} \kappa>0$ has integral kernel $\frac{1}{4 \pi|x-y|} e^{-\kappa|x-y|}$. Suppose that

$$
\frac{1}{4 \pi} \int \frac{|V(x)||V(y)|}{|x-y|^{2}} d^{3} x d^{3} y \equiv\|V\|_{R}^{2}<\infty
$$

called the Rollnik class in [543] after [501]. Then the Hilbert-Schmidt norm $\left\||V|^{1 / 2}\left(H_{0}+\kappa^{2}\right)^{-1}|V|^{1 / 2}\right\|_{\mathrm{HS}} \leq\|V\|_{R}$, so, by (14.7) $|V|^{1 / 2}$ is $H_{0}$-smooth with $\left\||V|^{1 / 2}\right\|_{H_{0}} \leq \pi^{-1}\|V\|_{R}^{1 / 2}$. If $V \in L^{3 / 2}\left(\mathbb{R}^{3}\right)$, the HLS inequality [586, Theorem 6.2.1, 409, [156] implies that $V$ is Rollnik.

Smoothness has an immediate consequence for the spectral type of $H$. 
Theorem 14.4 ([322]). Let $H$ be a self-adjoint operator and let $A$ be $H$-smooth. Then $\operatorname{ran}\left(A^{*}\right) \subset \mathcal{H}_{\mathrm{ac}}(H)$. In particular, if $\operatorname{ker}(A)=\{0\}$, then $H$ has purely a.c. spectrum.

The proof is very easy. If $d \nu$ is the $H$-spectral measure for $A^{*} \varphi$, then (14.6) says that

$$
\nu(I) \leq\|A\|_{H}\|\varphi\|^{2}|I|
$$

(where $|\cdot|$ is Lebesgue measure) for open intervals, I. By taking unions and using outer regularity, (14.9) holds for all sets, so $\nu$ is absolutely continuous.

Smoothness also implies existence and completeness of wave operators.

Theorem 14.5 ([322 ). Let $H, H_{0}$ be two self-adjoint operators. Let $A, B$ be closed operators so that $A$ is $H$-smooth and $B$ is $H_{0}$-smooth and so that

$$
H-H_{0}=A^{*} B
$$

in the sense that for $\psi \in D(H)$ and $\varphi \in D\left(H_{0}\right)$, we have that

$$
\langle H \psi, \varphi\rangle-\left\langle\psi, H_{0} \varphi\right\rangle=\langle A \psi, B \varphi\rangle .
$$

Then $\Omega^{ \pm}\left(H, H_{0}\right)$ exist and are complete.

Remarks. (1) Since smoothness implies relative boundedness, if $\psi \in D(H)$ and $\varphi \in D\left(H_{0}\right)$, then the right side of (14.11) makes sense.

(2) In some applications, one assumes that $H-H_{0}=\sum_{j=1}^{n} A_{j}^{*} B_{j}$ with each $A_{j}$ $H$-smooth and each $B_{j}$ is $H_{0}$-smooth. The proof in Remark 3 extends to this case or, alternatively, one can define smoothness for closed operators, $A$, from $\mathcal{H}$, the space on which $H$ is defined to $\mathcal{K}$, a perhaps distinct Hilbert space, and then pick $\mathcal{K}=\oplus_{j=1}^{n} \mathcal{H}, B=\oplus_{j=1}^{n} B_{j}, A=\oplus_{j=1}^{n} A_{j}$ so $A^{*} B=\sum_{j=1}^{n} A_{j}^{*} B_{j}$.

(3) The proof is again easy (indeed, one of the beauties of Kato smoothness theory is how much one gets with simple proofs). If $\psi \in D(H)$ and $\varphi \in D\left(H_{0}\right)$, $W(t)=e^{+i t H} e^{-i t H_{0}}$, then for $s<t$,

$$
\begin{aligned}
|\langle\psi,(W(t)-W(s)) \varphi\rangle| & =\left|\int_{s}^{t}\left\langle A e^{-i u H} \psi, B e^{-i u H_{0}} \varphi\right\rangle d u\right| \\
& \leq\left(\int_{-\infty}^{\infty}\left\|A e^{-i u H} \psi\right\|^{2} d u\right)^{1 / 2}\left(\int_{-s}^{t}\left\|B e^{-i u H_{0}} \varphi\right\|^{2} d u\right)^{1 / 2} \\
& \leq \sqrt{2 \pi}\|A\|_{H}\|\psi\|\left(\int_{-s}^{t}\left\|B e^{-i u H_{0}} \varphi\right\|^{2} d u\right)^{1 / 2}
\end{aligned}
$$

so

$$
\|(W(t)-W(s)) \varphi\| \leq \sqrt{2 \pi}\|A\|_{H}\left(\int_{-s}^{t}\left\|B e^{-i u H_{0}} \varphi\right\|^{2} d u\right)^{1 / 2}
$$

is Cauchy. Therefore, $\Omega^{ \pm}\left(H, H_{0}\right)$ exists. Since $H_{0}-H=-B^{*} A$, we conclude that they are also complete by Theorem 13.1 
We say that a closed operator, $A$ is $H$-supersmooth if and only if

$$
\|A\|_{H, \mathrm{SS}}^{2} \equiv \sup _{z \in \mathbb{C} \backslash \mathbb{R}}\left\|A(H-z)^{-1} A^{*}\right\|<\infty .
$$

The notion is in 322 and the name is from 352 in 1989. The name has not stuck but I like it, so I will use it. The fourth important result in 322 is the following.

Theorem 14.6 ([322]). Let $H_{0}$ be a self-adjoint operator. Let $A$ be $H_{0^{-}}$ supersmooth and $C$ a bounded self-adjoint operator so that

$$
\alpha \equiv\|C\|\|A\|_{H_{0}, \mathrm{SS}}^{2}<1 .
$$

Let $B=A^{*} C A$. Then $B$ is relatively form bounded with relative form bound at most $\alpha$. If $H=H_{0}+B$, then $A$ is also $H$-supersmooth with

$$
\|A\|_{H, \mathrm{SS}} \leq\|A\|_{H_{0}, \mathrm{SS}}(1-\alpha)^{-1 / 2} .
$$

In particular, $\Omega^{ \pm}\left(H, H_{0}\right)$ exist and are complete.

Remarks. (1) Once again, the proofs are simple. The key is a formal geometric series:

$$
\begin{aligned}
A(H-z)^{-1} A^{*}= & A\left(H_{0}-z\right)^{-1} A^{*}+\sum_{j=0}^{\infty}(-1)^{j+1} A\left(H_{0}-z\right)^{-1} A^{*} \\
& \times\left[C A\left(H_{0}-z\right)^{-1} A^{*}\right]^{j} C A\left(H_{0}-z\right)^{-1} A^{*} .
\end{aligned}
$$

One proves the form boundedness and uses that to justify a formula like (14.16) but with an error term. Since $\left\|C A\left(H_{0}-z\right)^{-1} A^{*}\right\| \leq \alpha$, the error goes to zero and the series converges. The final assertion then comes from Theorem 14.5

(2) By the same analysis, the analog of Remark (2) after Theorem 14.5 holds. If $H=H_{0}+\sum_{j=1}^{n} A_{j}^{*} B_{j}$ and $\gamma_{j k}=\sup _{z \in \mathbb{C} \backslash \mathbb{R}}\left\|B_{j}\left(H_{0}-z\right)^{-1} A_{k}^{*}\right\|$ is finite and $\Gamma=\left\{\gamma_{j k}\right\}_{1 \leq j, k \leq n}$ is a matrix of norm $\alpha<1$, and if each $A_{j}$ and $B_{j}$ is supersmooth, then $\Omega^{ \pm}\left(H, H_{0}\right)$ exist and are complete.

(3) We repeat that in 322 , Kato considers cases where $H_{0}$ and $C$ need not be self-adjoint. He assumes that $\sigma\left(H_{0}\right) \subset \mathbb{R}$ and $\|C\| \sup _{z}\left\|A\left(H_{0}-z\right)^{-1} A^{*}\right\|<1$ and then defines an operator $H$ which is formally $H_{0}+A^{*} C A$ with a resolvent that obeys (14.16). He then uses ideas going back to Friedrichs [166] to define (in terms of resolvents, not time limits) invertible operators $W^{ \pm}$so that $W^{ \pm} H_{0}\left(W^{ \pm}\right)^{-1}=H$.

That completes our discussion of 322]. The main result of 324] is the following.

Theorem 14.7 (Putnam-Kato Theorem [470, 324]). Let $A$ and $B$ be bounded self-adjoint operators so that $D \equiv i[A, B]$ is strictly positive in the sense that for all $\varphi \neq 0$, we have that

$$
\langle\varphi, D \varphi\rangle>0
$$

Then $A$ and $B$ have purely a.c. spectrum. 
Remarks. (1) The result is due to Putnam. Kato found the real simple proof in the next remark.

(2) The proof is easy. For let $C$ be the square root of $i[A, B]$. Then $\frac{d}{d t}\left\langle e^{-i t A} \varphi, B e^{-i t A} \varphi\right\rangle^{2}=\left\|C e^{-i t A} \varphi\right\|^{2}$ so the integral of $\left\|C e^{-i t A} \varphi\right\|^{2}$ from $s$ to $t$ is bounded by $2\|B\|\|\varphi\|$, Thus $C$ is $A$-smooth and $A$ has only a.c. spectrum on the closure of $\operatorname{ran}(C)$ which is all of $\mathcal{H}$.

Example 14.8 (Weak coupling 2-body). In [322, Kato applied smoothness ideas to Schrödinger operators. If $\nu=3$, as we have seen in Example 14.3. if $V \in L^{3 / 2}$ (indeed, if $V$ is Rollnik), then $|V|^{1 / 2}$ is $-\Delta$-supersmooth, so for small real $\lambda$, the wave operators, $\Omega^{ \pm}(-\Delta+\lambda V,-\Delta)$ exist and are unitary. On $(0, \infty)$, if $h_{0}=-\frac{d^{2}}{d x^{2}}$ with $u(0)=0$, then $\left(h_{0}-z\right)^{-1}$ has an integral kernel dominated by $\min (x, y)$ (see [587, (7.9.53)]) for all $z \in \mathbb{C} \backslash \mathbb{R}$, so if $\int_{0}^{\infty} x|V(x)| d x<\infty$, then $|V|^{1 / 2}$ is $h_{0}$-supersmooth and one knows that for $\lambda$ small, that $\Omega^{ \pm}\left(h_{0}+\lambda V, h_{0}\right)$ exists and are unitary.

One knows that if $\nu=1$ or 2 and $V \in C_{0}^{\infty}\left(\mathbb{R}^{\nu}\right) ; V \not \equiv 0$, then for all $\lambda \neq 0$, either $-\Delta+\lambda V$ or $-\Delta-\lambda V$ (or both) have a negative energy bound state [554] so there cannot be $-\Delta$-supersmoothness.

By interpolating between $\left\|e^{i t \Delta} \varphi\right\|_{\infty} \leq(4 \pi t)^{-\nu / 2}\|\varphi\|_{1}$ and $\left\|e^{i t \Delta} \varphi\right\|_{2}=\|\varphi\|_{2}$, Kato 322] showed that if $\nu \geq 4$ and $V \in L^{\nu / 2+\epsilon} \cap L^{\nu / 2-\epsilon}$, then $|V|^{1 / 2}$ is $-\Delta$-supersmooth and he conjectured that this held for $\epsilon=0$. Indeed, the next theorem is true.

Theorem 14.9. Let $\nu \geq 3$ and $V \in L^{\nu / 2}\left(\mathbb{R}^{\nu}\right)$. Then $V$ is supersmooth. In particular, for $|\lambda|$ small and $H=-\Delta+\lambda V, H_{0}=-\Delta$, we have that $\Omega^{ \pm}\left(H, H_{0}\right)$ exist and are unitary so that $H$ has purely a.c. spectrum.

Remarks. (1) This result appeared in [352. As they added in a "Note added in proof", shortly before their paper, Kenig-Ruiz-Sogge 353. proved estimates that imply Theorem 14.9

(2) In 259, Iorio-O'Carroll used supersmoothness to show that $N$-body systems with weak coupling (and $\nu \geq 3$ ) have unitary wave operators (so no bound states, no nontrivial scattering channels and purely a.c. spectrum). They required that the two body potentials lie in $L^{\nu / 2+\epsilon} \cap L^{\nu / 2-\epsilon}$, but given Theorem 14.9, their method works for two body potentials in $L^{\nu / 2}$.

Kato-Yajima 352 also proved that $\left(1+|x|^{2}\right)^{-1 / 2}(1-\Delta)^{1 / 4}$ is $-\Delta$-supersmooth (which says something about $V(x)=|x|^{-2}$ on $L^{2}\left(\mathbb{R}^{\nu}\right) ; \nu \geq 3$ ). Further developments are due to Ben et al. [44] and Simon [575]. In particular, Simon obtained optimal constants in the associated smoothness estimates; for $\nu \geq 3$,

$$
\begin{aligned}
& \int_{-\infty}^{\infty}\left\|\left(x^{2}+1\right)^{-1 / 2}(-\Delta)^{1 / 4} e^{i t \Delta} \varphi\right\|^{2} d t \leq \frac{\pi}{2}\|\varphi\|^{2}, \\
& \int_{-\infty}^{\infty}\left\||x|^{-1} e^{i t \Delta} \varphi\right\|^{2} d t \leq \frac{\pi}{\nu-2}\|\varphi\|^{2} .
\end{aligned}
$$




\section{B. Simon}

Next, having completed our discussion of Kato's contributions to smoothness, we turn some applications beginning with repulsive potentials. In this (and other) regard, it is useful to have the notion of local smoothness due to Lavine [398. Let $\Omega \subset \mathbb{R}$ be a bounded Borel set. We say that $A$ is locally $H$-smooth on $\Omega$ if $A P_{\Omega}(H)$ is $H$-smooth (where $P_{X}(H)$ is a spectral projection for $H$ and set $X$ [587, Sec. 5.1]). It is easy to see [479, Theorem XIII.30] that if $A$ is an operator with $D(H) \subset D(A)$ and either $\sup _{0< \pm \epsilon<1 ; \lambda \in \Omega} \epsilon\|A R(\lambda+i \epsilon)\|<\infty$ or $\sup _{0<\epsilon<1 ; \lambda \in \Omega}\left\|A R(\lambda+i \epsilon) A^{*}\right\|<\infty$, then $A$ is locally $H$-smooth on $\Omega$. It is also obvious that if $\operatorname{ran}\left(A^{*}\right)$ is dense, then, if $A$ is locally $H$-smooth, $H\left\lceil\operatorname{ran} P_{\Omega}(H)\right.$ is purely absolutely continuous. The following is what makes local $H$-smoothness so useful.

Theorem 14.10 ([398]). Let $H$ and $H_{0}$ be self-adjoint and $\Omega \subset \mathbb{R}$ a bounded open set. Suppose that $H=H_{0}+A^{*} B$, where $B$ is $H_{0}$-bounded and locally $H_{0}$-smooth on $\Omega$ and $A$ is $H$-bounded and locally $H$-smooth on $\Omega$. Then

$$
\Omega^{ \pm}\left(H, H_{0} ; P_{\Omega}\left(H_{0}\right)\right)=s-\lim _{t \rightarrow \mp \infty} e^{i t H} e^{-i t H_{0}} P_{\Omega}\left(H_{0}\right)
$$

exist and have range $P_{\Omega}(H)$.

Remarks. (1) For complete proofs, see [398] or [479, Theorem XIII.31].

(2) The same proof as Theorem 14.5 shows that $s-\lim _{t \rightarrow \mp \infty} P_{\Omega}(H) e^{i t H} e^{-i t H_{0}}$ $P_{\Omega}\left(H_{0}\right)$ exists.

(3) Since $B e^{-i t H_{0}} P_{\Omega}\left(H_{0}\right)\left(H_{0}-z\right)^{-1} \varphi$ is in $L^{2}$ with an $L^{2}$ derivative, we conclude that for any $z \in \mathbb{C} \backslash \mathbb{R}$,

$$
s^{-} \lim _{t \rightarrow \mp \infty} B e^{-i t H_{0}} P_{\Omega}\left(H_{0}\right)\left(H_{0}-z\right)^{-1}=0 .
$$

(4) Writing $(H-z)^{-1}-\left(H_{0}-z\right)^{-1}=\left[A(H-\bar{z})^{-1}\right]^{*} B\left(H_{0}-z\right)^{-1}$ and using the assumed boundedness of $A(H-\bar{z})^{-1}$, we conclude by Remark (3) that $s-\lim _{t \rightarrow \mp \infty}\left[(H-z)^{-1}-\left(H_{0}-z\right)^{-1}\right] e^{-i t H_{0}} P_{\Omega}\left(H_{0}\right)=0$ and then by [98. Appendix to Chap. 3] that $s-\lim _{t \rightarrow \mp \infty}\left[f(H)-f\left(H_{0}\right)\right] e^{-i t H_{0}} P_{\Omega}\left(H_{0}\right)=0$ for any continuous function, $f$, so that $1-f$ has compact support. Using this, one sees if $I \subset \Omega$ is a compact set with $\operatorname{dist}(I, \mathbb{R} \backslash \Omega)>0$, then $s$ - $\lim _{t \rightarrow \mp \infty} P_{\mathbb{R} \backslash \Omega} e^{i t H} e^{-i t H_{0}} P_{I}\left(H_{0}\right)=0$. This implies that the limits in (14.20) exist and that $\operatorname{ran} \Omega^{ \pm}\left(H, H_{0} ; P_{\Omega}\left(H_{0}\right)\right) \subset \operatorname{ran} P_{\Omega}(H)$. This plus symmetry between $H$ and $H_{0}$ plus the idea behind Theorem 13.1 imply that $\operatorname{ran} \Omega^{ \pm}\left(H, H_{0} ; P_{\Omega}\left(H_{0}\right)\right)=\operatorname{ran} P_{\Omega}(H)$.

A potential, $V$, on $\mathbb{R}^{\nu}$ is called repulsive if and only if $\mathbf{x} \cdot \nabla V \leq 0$ (e.g. $V(x)=$ $(1+|x|)^{-\alpha}$, any $\left.\alpha>0\right)$. If $V(x) \rightarrow 0$ at infinity, then $V(x) \geq 0$. If $A=\frac{i}{2}(\mathbf{x} \cdot \nabla+\nabla \cdot \mathbf{x})$ is the generator of dilations and $V$ is repulsive, then $i\left[A, H_{0}+\right.$ $V]=2 H_{0}-\mathbf{x} \cdot \nabla V \geq 0$. One cannot use the Putnam-Kato theorem since neither $A$ nor $H$ is bounded. If you look at the above proof of the Putnam-Kato theorem, that $H$ is unbounded is not a problem if our goal is to find a $C$ which is $H$-smooth. But the unbounded $A$ is. Lavine's idea was to cutoff $\mathbf{x}$ in the definition of $A$ and get an $\tilde{A}$ which is $H$-bounded and so that $i[\tilde{A}, H] \geq c\left(1+|x|^{2}\right)^{-\beta}$ for suitable $\beta$ and as in the Putnam-Kato argument, get that $\left(1+|x|^{2}\right)^{-\beta / 2}(H+1)^{-1}$ is $H$-smooth. 
In this way (he used local smoothness to get wave operators), Lavine proved the following.

Theorem 14.11 ([396-399]). Let $H$ be an $N$-body Hamiltonian with center of mass removed on $L^{2}\left(\mathbb{R}^{(N-1) \nu}\right)$ whose two body potentials $V_{i j}$ lie in $L^{p}\left(\mathbb{R}^{\nu}\right)+L^{\infty}\left(\mathbb{R}^{\nu}\right)$ (with $p$ $\nu$-canonical) and are repulsive. Then $H$ has purely absolutely continuous spectrum. If moreover, for some $\beta>5 / 2$, we have that $\left|V_{i j}(x)\right| \leq C(1+|x|)^{-\beta}$, then $\Omega^{ \pm}\left(H, H_{0}\right)$ exist and are complete.

Remark. $5 / 2$ is an artifact of the proof and when the $V_{i j}$ are spherically symmetric, it has been improved to $\beta>1$ in 399 .

Our final major topic concerns ideas of Vakulenko [626]; the reader should first look at the discussion around Eq. (12.6) for definitions of Vakulenko bounding function and Vakulenko potential.

Lemma 14.12 ([626]). Let $H$ be self-adjoint and $A$ a closed $H$-bounded operator. Let $[a, b]$ be a bounded closed interval in $\mathbb{R}$ and $B$ a closed operator with $D(H) \subset$ $D(B)$ so that for all $\varphi \in D(H)$ and $\lambda \in[a, b]$, we have that

$$
\operatorname{Re}\langle(H-\lambda) \varphi, A \varphi\rangle \geq\|B \varphi\|^{2} .
$$

Then $B$ is $H$-smooth on $[a, b]$.

Remarks. (1) As a preliminary, we note that since $\frac{|x-\lambda|}{|x-(\lambda+i \epsilon)|} \leq 1$, we have that

$$
\|(H-\lambda) R(\lambda+i \epsilon)\| \leq 1
$$

(2) As a second preliminary, if

$$
\|A \varphi\| \leq \alpha\|H \varphi\|+\beta\|\varphi\|
$$

then

$$
\begin{aligned}
\|A R(\lambda+i \epsilon) \psi\| & \leq \alpha\|[(H-\lambda)+\lambda] R(\lambda+i \epsilon) \psi\|+\beta\|R(\lambda+i \epsilon) \psi\| \\
& \leq\left(\alpha+\alpha|\lambda| \epsilon^{-1}+\beta \epsilon^{-1}\right)\|\psi\| .
\end{aligned}
$$

(3) Letting $\varphi=R(\lambda+i \epsilon) \psi$ in (14.21), we see that

$$
\begin{aligned}
\|B R(\lambda+i \epsilon) \psi\|^{2} & \leq\|(H-\lambda) R(\lambda+i \epsilon)\|\|A R(\lambda+i \epsilon)\|\|\psi\|^{2} \\
& \leq C \epsilon^{-1}\|\psi\|^{2}
\end{aligned}
$$

(by (14.22) / 14.24) ) for $0<\epsilon<1$ and all $\lambda \in[a, b]$, where $C$ is a constant depending on $\alpha, \beta, a$ and $b$. This implies local smoothness by the discussion prior to Theorem 14.10

(4) Vakulenko's $A$ is close to $i$ times a cutoff dilation generator, so the left side of (14.21) is like an expectation of a commutator and thus this is a variant of a Mourre estimate but unlike the Mourre estimate, there is no (compact) error term. 
In Theorem 12.2, we stated a bound of the form 14.21 which immediately implies (given the lemma) the following.

Theorem $14.13([626])$. Let $V(x)$ be a Vakulenko potential with (12.7) for some Vakulenko bounding function $\eta$. Then $\sqrt{\eta}$ is $-\Delta+V$ locally smooth on $(0, \infty)$. In particular, the spectrum of $-\Delta+V$ is purely absolutely continuous on $(0, \infty)$ and the wave operators exist and are complete.

Remarks. (1) Since $\eta$ is everywhere non-vanishing, ran $\sqrt{\eta}$ is dense and this implies the absolute continuity on $(0, \infty)$.

(2) $\sqrt{\eta}$ is locally smooth for both $-\Delta+V$ and $-\Delta$ (since the zero potential is a Vakulenko potential with bounding function $\eta$ ). Since $|V|^{1 / 2} \leq \sqrt{\eta}$, we see that $|V|^{1 / 2}$ is locally smooth which implies that wave operators exist and are complete.

(3) The proof of Theorem 12.2 is particularly easy when $\nu=1$. Fix $\lambda_{0}>0$ and let

$$
\omega(x)=\exp \left[\frac{2}{\sqrt{\lambda_{0}}} \int_{-\infty}^{x} \eta(y) d y\right]
$$

and

$$
A=2 \omega \frac{d}{d x} .
$$

Since $\eta \in L^{1}(\mathbb{R}), \omega$ is bounded so since $\frac{d}{d x}(-\Delta+V+i)^{-1}$ is bounded, we see that $A$ is $H$-bounded. It is easy to see (since $\eta$ and $V$ are real) that it suffices to prove (14.21) when $\varphi$ is real in which case

$$
\langle(H-\lambda) \varphi, A \varphi\rangle=\int_{-\infty}^{\infty}\left[\omega^{\prime}\left[\left(\varphi^{\prime}\right)^{2}+\lambda(\varphi)^{2}\right]+2 \omega V \varphi \varphi^{\prime}\right] d x
$$

which we get by integration by parts in

$$
2 \int_{-\infty}^{\infty}\left(-\varphi^{\prime \prime}-\lambda \varphi\right) \omega \varphi^{\prime} d x=-\int_{-\infty}^{\infty} \omega\left[\left(\varphi^{\prime}\right)^{2}+\lambda(\varphi)^{2}\right]^{\prime} d x
$$

Since $\left(\left|\varphi^{\prime}\right|-\sqrt{\lambda} \varphi\right)^{2}=\left(\varphi^{\prime}\right)^{2}+\lambda(\varphi)^{2}-2 \sqrt{\lambda}\left|\varphi^{\prime}\right||\varphi|$ we see that

$$
\text { Right-hand side of (14.28) } \geq \int_{-\infty}^{\infty}\left(\omega^{\prime}-\frac{|V(x)|}{\sqrt{\lambda}} \omega\right)\left[\left(\varphi^{\prime}\right)^{2}+\lambda(\varphi)^{2}\right]^{\prime} d x \text {. }
$$

By construction of $\omega,|V| \leq \eta, \omega \geq 1$ and $\lambda>\lambda_{0}$, we have that

$$
\omega^{\prime}-\frac{|V(x)|}{\sqrt{\lambda}} \omega \geq \frac{1}{\sqrt{\lambda_{0}}} \omega \eta \geq \frac{\eta}{\sqrt{\lambda_{0}}} .
$$

Thus

$$
\begin{aligned}
\text { Right-hand side of }(14.29) & \geq \int_{-\infty}^{\infty} \frac{\lambda}{\sqrt{\lambda_{0}}} \eta(x)(\varphi)^{2} d x \\
& \geq \sqrt{\lambda_{0}}\|\sqrt{\eta} \varphi\|^{2}
\end{aligned}
$$

which is (14.21). The higher-dimensional case needs a carefully constructed $\omega$ but is along similar lines. 
(4) Since $\eta(x)=(1+|x|)^{-\alpha}, \alpha>1$ is a Vakulenko bounding function, we get the Corollary as follows.

Corollary 14.14. If

$$
|V(x)| \leq C(1+|x|)^{-\alpha}
$$

for some $\alpha>1$, then $H=-\Delta+V$ has purely a.c. spectrum on $(0, \infty)$ and with $H_{0}=-\Delta, \Omega^{ \pm}\left(H, H_{0}\right)$ exist and are complete.

Thus Vakulenko obtained a new and beautiful proof of an Agmon-KatoKuroda-type theorem of the kind we discuss in the next section (albeit 15 years after their work). Unlike their method, this one seems to require pointwise bounds and does not allow for local singularities.

Yafaev 665] has an approach to long-range two-body scattering that exploits some ideas from the theory of smooth perturbations.

We note that the earliest proofs of $N$-body asymptotic completeness for $0\left(|x|^{-1-\epsilon}\right.$ ) potentials (at least when $N \geq 4$ ) were by Sigal-Soffer [537, 538] and then by Graf [190] and Dereziński [112. [112] and [538] have results on long-range results. In 664], Yafaev found a proof that exploits smoothness ideas (as well as some of the tools - Mourre estimates 431, 465, 171, Deift-Simon wave operators [107, Enss-type phase space analysis [136, 137] — of the earlier approaches). Kato never considered $N$-body scattering, which is quite involved, so we refer the reader to Yafaev's original paper 664 or lecture notes [667 for details.

\section{Scattering and Spectral Theory, III: Kato-Kuroda Theory}

This is the third section on spectral and scattering theory; it focuses on stationary, aka time-independent, methods. As with the prior two sections, we will include an overview portion but we want to begin by describing the problem we will discuss and the contributions of Agmon, Kato and Kuroda. While it is significant that local singularities can be accommodated, we will mainly discuss the case (13.16), i.e.

$$
|V(x)| \leq C(1+|x|)^{-\alpha} .
$$

We consider $H_{0}=-\Delta, H=-\Delta+V(x)$ on $L^{2}\left(\mathbb{R}^{\nu}, d^{\nu} x\right)$. These are the questions that will concern us:

(A) Existence and Completeness of $\Omega^{ \pm}\left(H, H_{0}\right)$.

(B) Absence of singular continuous spectrum.

As a sidelight of the methods, one also gets continuum eigenfunction expansions of a type I will discuss as follows. There is also the issue of positive eigenvalues which except for the work of Vakulenko (as discussed in Secs. 12 and 14) was studied using very different methods from those used in this section; see Sec.12.

As we explained in Sec.13, it follows from Cook's method that $\Omega^{ \pm}\left(H, H_{0}\right)$ exist if $\alpha>1$ while they may not if $\alpha \leq 1$. It is known (see Sec. 20) that (B) can fail if 
$\alpha<1$ (although this was not known in the 1970s), so in the 15 years after 1957 , a lot of effort was made on studying problems (A) and (B) when $\alpha>1$. We will say a lot more about the detailed history later but start with the best results of Kato-Kuroda on the subject and on the optimal result.

In 1969, Kato 325] using, in part, ideas of Kato-Kuroda (of which we will say a lot more below) proved the following.

Theorem 15.1 ([325]). Let $V$ obey (15.1]) and $H, H_{0}$ as above. Then

(a) If $\alpha>1$, the wave operators exist and are complete.

(b) If $\alpha>5 / 4, H$ has no singular continuous spectrum.

In 1970, Agmon [3] announced the following.

Theorem 15.2 ([3, 4]). Let $V$ obey [15.1) and $H$ as above. If $\alpha>1, H$ has no singular continuous spectrum.

While Agmon did not discuss scattering in his announcement, Lavine 400] noted that his estimates and Lavine's theory of local smoothness implied existence and completeness of wave operators (and later, both Agmon and Hörmander presented other approaches to get completeness). We also note that as discussed, for example, in [479. Sec. XIII.8], one can accommodate local singularities; in place of assuming $(1+|x|)^{\alpha} V(x)$ is bounded, one needs only to assume that it is a relatively compact perturbation of $-\Delta$.

Agmon was able to go from $5 / 4$ to 1 by an astute observation (Step 8 in the scheme at the end of the section). By using the same idea, Kuroda could extend that Kato-Kuroda argument up to $\alpha>1$. Later we will say more about work of others on these problems.

Our goal in this section is to explain the machinery behind certain proofs of Theorems 15.1 and 15.2 We begin with some general overview of the stationary approach to scattering. The earliest mathematical approach to stationary scattering is in [166] but we will focus on a slightly later one of Povzner [468] in 1953 and Kato's student, Ikebe [252], in 1960 that discusses eigenfunction expansion. Their expansions are to be distinguished from what 571] calls BGK expansions after Berezanskii, Browder, Gårding, Gel'fand and Kac (see references in [571]). The BGK expansion is essentially a variant of the spectral theorem when an operator $A$ on $L^{2}\left(\mathbb{R}^{\nu}, d^{\nu} x\right)$ has local trace class properties (i.e. $f(x) P_{[a, b]}(A) f(x)$ is trace class for $\left.f \in C_{0}^{\infty}\left(\mathbb{R}^{\nu}\right)\right)$. This expansion is stated in terms of the spectral measures and so has no implications for the spectral properties. The advantage of BGK expansions is that they are always applicable for Schrödinger operators (see [571]) while the Povzner-Ikebe expansion only works in special situations, but when it does, it provides a lot of additional information.

The IP expansion of Povzner-Ikebe involves not spectral measures but $d^{\nu} k$ which is why it has important spectral consequences. The model is the Fourier transform for $H_{0}=-\Delta$ which in this introductory discussion, we will denote as $\hat{f}_{0}$ (since we 
will use $\hat{f}$ for something else) defined on $\mathbb{R}^{\nu}$ by

$$
\begin{aligned}
\hat{f}_{0}(\mathbf{k}) & =(2 \pi)^{-\nu / 2} \int \overline{\varphi_{0}(\mathbf{x}, \mathbf{k})} f(\mathbf{x}) d^{\nu} x, \\
\varphi_{0}(\mathbf{x}, \mathbf{k}) & =e^{i \mathbf{k} \cdot \mathbf{x}}
\end{aligned}
$$

(see [583 Sec. 6.5] for the meaning of (15.2) when $f$ is only in $L^{2}$ and not in $L^{1}$ ). This provides an eigenfunction expansion of $H_{0}$ in that (except for places where we want to emphasize the vector nature of $\mathbf{x}$ and $\mathbf{k}$, we will start using non-boldface)

$$
\begin{aligned}
& f(x)=(2 \pi)^{-\nu / 2} \int \varphi_{0}(x, k) \hat{f}_{0}(k) d^{\nu} k, \\
& \widehat{H_{0} f_{0}}(k)=|k|^{2} \hat{f}_{0}(k),
\end{aligned}
$$

so that formally (and much more), $H_{0} \varphi(\cdot, k)=|k|^{2} \varphi(\cdot, k)$.

For suitable $V$ and $H=H_{0}+V$, what Povzner and Ikebe found are functions, $\varphi(\mathbf{x}, \mathbf{k})$, so that if $\hat{f}$ is defined by

$$
\hat{f}(k)=(2 \pi)^{-\nu / 2} \int \overline{\varphi(x, k)} f(x) d^{\nu} x
$$

and if $\left\{\varphi_{n}(x)\right\}_{n=1}^{N}$ is an orthonormal basis of $L^{2}$ eigenfunctions for $\mathcal{H}_{\mathrm{pp}}(H)$ with $H \varphi_{n}=E_{n} \varphi_{n}$, then

$$
f(x)=\sum_{n=1}^{N}\left\langle\varphi_{n}, f\right\rangle \varphi_{n}(x)+(2 \pi)^{-\nu / 2} \int \varphi(x, k) \hat{f}(k) d^{\nu} k
$$

and

$$
\widehat{H f}(k)=|k|^{2} \hat{f}(k) .
$$

This implies that $H$ has point spectrum plus a.c. spectrum solving problem (B).

They also proved a connection to scattering

$$
\widehat{\Omega^{+} f}=\hat{f}_{0}
$$

so that formally

$$
\Omega^{+} \varphi=\varphi_{0}
$$

(we will say more about this shortly). This implies that $\operatorname{ran} \Omega^{+}=\mathcal{H}_{\mathrm{ac}}(H)$ and then, since $\Omega^{+} \bar{f}=\overline{\Omega^{-} f}$ (where - is complex conjugate), we have that ran $\Omega^{+}=\mathcal{H}_{\mathrm{ac}}(H)$ solving problem $(\mathrm{A})$.

In the physics literature, Gell'Mann and Goldberger, [179] appealing to stationary phase arguments [585, Sec. 15.3], considered the meaning of (15.10) and formally proved that pointwise it held if the limit in the definition of wave operator is an abelian limit (i.e. an $e^{-\epsilon t}$ is added to the quantity in the limit and then one takes $\epsilon \downarrow 0)$. Indeed, Ikebe proved (15.9) in terms of abelian limits and then used the existence of the ordinary limit proven by other means. 


\section{B. Simon}

Of course, one has to find suitable continuum eigenfunctions, $\varphi(\mathbf{x}, \mathbf{k})$, so that (15.9) holds. Some thought about Born's ideas suggests that one wants $\varphi$ to have the asymptotics (13.1) near $\mathbf{x}=\infty$. We will explain that $\varphi$ obeys an integral equation called the Lippmann-Schwinger equation introduced by two physicists [415] in 1950. Following Lippmann-Schwinger, Povzner and Ikebe, we only consider $\nu=3$ where the integral kernel for $\left(H_{0}-k^{2}\right)^{-1}$ is especially simple.

Since formally $\left(H_{0}+V-k^{2}\right) \varphi=0$, we might expect that $\varphi$ obeys $\varphi=-\left(H_{0}-\right.$ $\left.k^{2}\right)^{-1} V \varphi$. There are two problems with this. First, since $k^{2}$ is in the spectrum of $H_{0}$, we cannot use $\left(H_{0}-k^{2}\right)^{-1}$ as a bounded operator on $L^{2}$. If $\operatorname{Im}(k)>0$ (so $\left.k^{2} \notin \mathbb{R}\right)$, then $\left(H_{0}-k^{2}\right)^{-1}$ has an integral kernel, $G_{0}\left(\mathbf{x}, \mathbf{y} ; k^{2}\right)$, given by

$$
G_{0}\left(x, y ; k^{2}\right)=\frac{e^{i k|x-y|}}{4 \pi|x-y|} .
$$

This has a pointwise limit as $k^{2} \rightarrow \mathbb{R}$, indeed two different limits if one takes $\epsilon \downarrow 0$ for $k^{2} \pm i \epsilon$. We thus define for $k>0$,

$$
G_{0}\left(x, y, k^{2} \pm i 0\right)=\frac{e^{ \pm i k|x-y|}}{4 \pi|x-y|} .
$$

As we will see, to get (15.9), we want to pick $+i 0$, not $-i 0$. It is the use of plus here that led physicists to use $\Omega^{+}$for the limit as $t \rightarrow-\infty$. This gives meaning to $-\left(H_{0}-k^{2}\right)^{-1} V \varphi$.

The second problem with $\varphi=-\left(H_{0}-k^{2}\right)^{-1} V \varphi$ is that if $V$ has rapid decay (e.g. $V$ has compact support), it is not hard to see that $-\left(H_{0}-k^{2}\right)^{-1} V \varphi$ looks like the second term in (13.1), so it is tempting to $\operatorname{try} \varphi=e^{i k . x}-\left(H_{0}-k^{2}\right)^{-1} V \varphi$. Note that since $\left(H_{0}-k^{2}\right)$ has a kernel (among "reasonable" functions), we are allowed to add elements of the kernel when inverting; put differently $\left(H_{0}-k^{2}\right)\left[e^{i k \cdot x}-\right.$ $\left(H_{0}-k^{2}\right)^{-1} V \varphi=-V \varphi$ and thus our formal eigenfunctions will be solutions of the Lippmann-Schwinger equation

$$
\varphi(\mathbf{x}, \mathbf{k})=e^{i \mathbf{k} \cdot \mathbf{x}}-\frac{1}{4 \pi} \int \frac{e^{i|\mathbf{k}||\mathbf{x}-\mathbf{y}|}}{|\mathbf{x}-\mathbf{y}|} V(\mathbf{y}) \varphi(\mathbf{y}) d^{3} y .
$$

The pioneer in using the Lippmann-Schwinger equation to prove mathematical results about eigenfunction expansions was Povzner [468, 469]. In [468], published in 1953, he considered $C^{\infty}$ potentials, $V$, obeying (15.1) for $\nu=3, \alpha>7 / 2$ and solved problem (B) affirmatively for such $\alpha$. In 1955, in [469, for $V$ 's of compact support, he solved problem (A) (when $\nu=3$ ). Bear in mind that the results of Cook, Hack and Kuroda on existence (via Cook's method) did not exist when Povzner wrote 469. As we will see, Ikebe's approach to solving problem (A) uses these a priori existence results.

In 1960, Ikebe 252 used eigenfunction expansions to solve problems (A) and (B) when $\nu=3$ and $V$ obeys (15.1) near infinity for $\alpha>2$ and moreover, $V$ is Hölder continuous away from a finite number of points where it is locally $L^{2}$. Let 
us sketch the ideas that he used:

(i) Let $B$ be the Banach space, $C_{\infty}\left(\mathbb{R}^{3}\right)$, of bounded functions vanishing at $\infty$ with $\|\cdot\|_{\infty}$. For $\operatorname{Im}(\kappa) \geq 0$, define

$$
\left(T_{\kappa} g\right)(x)=-\frac{1}{4 \pi} \int \frac{e^{i \kappa|x-y|}}{|x-y|} V(y) g(y) d^{3} y .
$$

Then if $V$ obeys (15.1) with $\alpha>2, T_{\kappa}$ is a bounded, indeed a compact, operator of $B$ to $B$ which is analytic in $\kappa$ on $\mathbb{C}_{+}$and Hölder continuous on $\overline{\mathbb{C}_{+}} \backslash\{0\}$.

(ii) One shows that $T_{\kappa} \psi=\psi$ has no nonzero solution for $\operatorname{Im}(\kappa)>0$ (since $\psi$ is then exponentially decreasing and so in $L^{2}$ violating self-adjointness) and then also for $\operatorname{Im}(\kappa)=0, \kappa \neq 0$ since one can use Kato's result mentioned in Remark 4 after Theorem 12.1. In this analysis, Ikebe shows that if $\kappa \in \mathbb{R} \backslash\{0\}$ and $\psi$ solves $T_{\kappa} \psi=\psi$, then $\varphi \equiv \psi \in L^{2}\left(\mathbb{R}^{3}\right)$ obeys

$$
\int_{|k|=\kappa}|\hat{\varphi}(k)|^{2} d \omega=0
$$

suitably interpreted. This result, also found by Povzner, is important as we will see later.

(iii) By Fredholm theory, since $T_{\kappa} \psi=\psi$ has no solutions, $\mathbf{1}-T_{\kappa}$ is invertible. One defines $\varphi(\cdot, \mathbf{k})$ to be $\left(\mathbf{1}-T_{|k|}\right)^{-1} \varphi_{0}(\cdot, \mathbf{k})$ with $\varphi_{0}$ given by $(\mathbf{1 5 . 3})\left(\varphi_{0} \notin B\right.$ since it does not vanish at infinity but if $\eta=\varphi-\varphi_{0}$, then $\varphi=\varphi_{0}+T_{|k|} \varphi \Leftrightarrow \eta=T_{|k|} \varphi+$ $T_{|k|} \eta$. Note that $\eta$ and $T_{|k|} \varphi_{0}$ are in $B$ ). In this way, one gets solutions of the Lippmann-Schwinger equation.

(iv) One also solves $G=G_{0}+T_{\kappa} G$ (where $G_{0}$ is the free Green's function (15.12) ) and uses this plus Stone's theorem to verify the expansion (15.7).

(v) By following arguments of Gell'Mann-Goldberger [179], one proves (15.9) where $\Omega^{+}$is an abelian limit. By the results of Cook-Hack-Kuroda, this abelian limit is equal to the ordinary limit.

(vi) 15.7) solves problem (B) and 15.9 solves problem (A) as noted above.

(vii) There is a gap in 252 found and filled in Simon 543 and also filled by Ikebe 253].

We should briefly mention two variants of Ikebe's work. First, Thoe 615 extended the result to $\mathbb{R}^{\nu}$ for general $\nu$. Second, for Rollnik potentials (any $V$ obeying (15.1) for $\alpha>2$ is in $L^{3 / 2}$ and so Rollnik but Rollnik allows $L^{3 / 2}$ local singularities), following Rollnik 501] and Grossman-Wu 202, one can rewrite the Lippmann-Schwinger equation in an equivalent form:

$$
\begin{aligned}
\xi(x) & =\xi_{0}(x)-\frac{1}{4 \pi} \int|V(x)|^{1 / 2} \frac{e^{i k|x-y|}}{|x-y|} V^{1 / 2}(y) \xi(y) \\
& \equiv \xi_{0}(x)+\left(W_{|k|} \xi\right)(x),
\end{aligned}
$$

where $V^{1 / 2}(y)=|V(y)|^{1 / 2} \operatorname{sgn}(V(y))$ and $\xi(x)=|V(x)|^{1 / 2} \varphi(x)$. The point is that the integral kernel in (15.16) is Hilbert-Schmidt for $\operatorname{Im}(k) \geq 0$ if $V$ is Rollnik. This was used by Simon [543] to carry over Ikebe's arguments. One big difference is that 
there is no Kato argument to eliminate solutions of the homogeneous equations. But by Fredholm theory, in any event, the set of points where $1-W_{|k|}$ is not invertible is the set of zeros of a function analytic on $\mathbb{C}_{+}$and continuous on its closure, so a subset of $\mathbb{R}$ with real Lebesgue measures zero. This allows a proof of completeness but not a solution of problem (B). We will say more about this issue as follows. We note that this factorization idea is used in several of the approaches to the Agmon-Kato-Kuroda theory and, in particular, an option in the work of Kato and Kuroda. We will not discuss this further.

Subsequent to Ikebe solving problem (B) if $\alpha>2$, the search for the general $\alpha>1$ result was solved in stages: Jäger [264] did it for $\alpha>3 / 2$, Rejto [481] for $\alpha>$ $4 / 3$, Kato 325] using Kato-Kuroda theory $\operatorname{did} \alpha>5 / 4$ as we have seen, Rejto [484] did $\alpha>6 / 5$ and finally Agmon [4] (and shortly afterwards, independently Saito [508]) handled $\alpha>1$. As we will explain using one simple idea from Agmon, Kuroda and Rejto could extend their methods to handle $\alpha>1$. Howland [241] had earlier work on this problem and Schechter [510] used Kato-Kuroda theory to study higherorder elliptic operators (as we will see Agmon, Hörmander and Kuroda also did).

In two papers [350, 349, Kato and Kuroda developed what they called an abstract theory of scattering. As Kuroda told me "it was too abstract to become popular" (blaming himself for this). In recognition of the history, Reed-Simon dubbed the basic result for $\alpha>1$ the Agmon-Kato-Kuroda Theorem but it is Agmon's approach that has stuck around. And this is not only due to the abstraction but also to the elegance and simplicity of Agmon's approach and its flexibility. Moreover, two early, widely used monograph presentations (Reed-Simon [479, Sec. XIII.8] and Hörmander [239, 240]) exposed the Agmon approach. All this said that, while Agmon's technicalities are distinct from Kato-Kuroda, the underlying conceptual framework is similar. We will describe this scheme using Agmon's approach to explicitly implement the steps.

Agmon uses the spaces $L_{\beta}^{2}\left(\mathbb{R}^{\nu}\right)$ defined by

$$
\|\varphi\|_{\beta}^{2}=\int\left(1+|x|^{2}\right)^{\beta}|\varphi(x)|^{2} d^{\nu} x<\infty .
$$

These are Hilbert spaces. One suppresses the natural duality of Hilbert spaces and associates the dual of $L_{\beta}^{2}$ with $L_{-\beta}^{2}$ so that $\psi \in L_{-\beta}^{2}$ is associated with the linear functional $\varphi \mapsto \int \overline{\psi(x)} \varphi(x) d^{\nu} x$. Here are the basic facts about Fourier transform on $L_{\beta}^{2}$ that we will need. For proofs, see [477, Sec. IX.9]; basically, one proves things for $\nu=1$ and uses spherical coordinates for the other variables. We return to using $f \mapsto \hat{f}$ for the Fourier transform.

(1) Let $\beta>1 / 2$. There is for each $\lambda \in(0, \infty)$, a bounded map, $T_{\lambda}: L_{\beta}^{2}\left(\mathbb{R}^{\nu}\right) \rightarrow$ $L^{2}\left(S^{\nu-1}, d \omega\right)$ (where $d \omega$ is unnormalized measure on the unit sphere in $\mathbb{R}^{\nu}$ ), so that if $f \in \mathcal{S}\left(\mathbb{R}^{\nu}\right)$, then

$$
\left(T_{\lambda} f\right)(\omega)=\widehat{f}(\lambda \omega) .
$$

(2) $T_{\lambda}$ is norm Hölder continuous in $\lambda$ of order $\beta-1 / 2$ if $1 / 2<\beta<3 / 2$. 
(3) Fix $\beta>1 / 2$. As maps of $L_{\beta}^{2}$ to $L_{-\beta}^{2},\left(-\Delta-\kappa^{2}\right)^{-1}$ defined initially for $\operatorname{Im} \kappa>0$ has a continuous extension to $\kappa \in \mathbb{R} \backslash\{0\}$.

(4) If $\varphi \in L_{\beta}^{2}, \beta>1 / 2$ and $\kappa>0$, then

$$
\lim _{\epsilon \downarrow 0} \operatorname{Im}\left\langle\varphi,\left(-\Delta-\left(\kappa^{2}+i \epsilon\right)\right)^{-1} \varphi\right\rangle=\frac{\pi \kappa^{\nu-2}}{2}\left\|T_{\kappa} \varphi\right\|_{2}^{2} .
$$

(This is just a version of $\lim _{\epsilon \downarrow 0} \frac{1}{x-i \epsilon}=\mathcal{P}\left(\frac{1}{x}\right)+i \pi \delta(x)$ ).

(5) Let $\beta>1 / 2$. Fix $\kappa>0$ and suppose that $\varphi \in L_{\beta}^{2}$ with $T_{\kappa} \varphi=0$. Define $Q_{\kappa} \varphi$ by

$$
\widehat{Q_{\kappa} \varphi}(\mathbf{k})=\left(k^{2}-\kappa^{2}\right)^{-1} \hat{\varphi}(\mathbf{k}) .
$$

Then for each $\epsilon>0, Q_{\kappa} \varphi \in L_{\beta-1-\epsilon}^{2}$ and

$$
\left\|Q_{\kappa} \varphi\right\|_{\beta-1-\epsilon} \leq C_{\epsilon, \kappa, \nu, \beta}\|\varphi\|_{\beta},
$$

where $C$ depends continuously on its parameters in the region $\epsilon, \kappa>0, \beta>1 / 2$. The point here is that without $T_{\kappa} \varphi=0$, we can define the limit as $\epsilon \downarrow 0$ of $\left(k^{2}-\kappa^{2}-i \epsilon\right)^{-1} \hat{\varphi}(\mathbf{k})$ which for $\varphi \in L_{\beta}^{2}$ with $\beta>1 / 2$ lies in $L_{-\beta}^{2}$ but we can never get better than $L_{-1 / 2}^{2}$. When $T_{\kappa} \varphi=0$, by having $\beta$ large, we can get $\varphi$ into a suitable $L_{\gamma}^{2}$ and, in particular, into $L^{2}$.

We can now describe the basic strategy of solving problems (A) and (B) for any $\alpha>1$.

Step 1. Find a triple of spaces $X \subset L^{2}\left(\mathbb{R}^{\nu}, d^{\nu} x\right) \subset X^{*}$, where $X$ is a dense subspace of $L^{2}$ and which is a Banach space in a norm, $\|\cdot\|_{X}$, so that for $\psi \in X$, we have that $\|\psi\|_{2} \leq\|\psi\|_{X}$. Any $\varphi \in L^{2}$ acts as a bounded linear functional on $X$ via $\ell_{\varphi}(\psi)=\langle\bar{\varphi}, \psi\rangle$ so $L^{2} \subset X^{*}$ which can be shown to be dense. Note that when $\varphi \in L^{2}$, we have that $\|\varphi\|_{X^{*}} \leq\|\varphi\|_{2}$. In the Agmon approach, $X=L_{\beta}^{2}\left(\mathbb{R}^{\nu}\right)$ for some $\beta>1 / 2$ and $X^{*}=L_{-\beta}^{2}\left(\mathbb{R}^{\nu}\right)$. Let $H_{0}$ be a self-adjoint operator which in the Agmon setup is a constant coefficient elliptic partial differential operator although we will mainly be interested in the case $H_{0}=-\Delta$. By the norm inequalities, for any $z \in \mathbb{C} \backslash\left[E_{0}, \infty\right)$ (where $E_{0}$ is the bottom of the spectrum of $\left.H_{0}\right),\left(H_{0}-z\right)^{-1}$ is bounded from $X$ to $X^{*}$. One must pick $X$ so that $\left(H_{0}-z\right)^{-1}$, as bounded maps from $X$ to $X^{*}$ has a continuous extension to $\left[E_{0}, \infty\right)$ with a finite set of points removed. The extension is from above or below the real axis and the two limits need not be equal. In our case where $E_{0}=0$, the finite set is only $E_{0}$. In the general elliptic case, it is the set of critical points of the defining symbol. As explained above, in the Agmon setup, where $X=L_{\beta}^{2}, \beta>1 / 2$, we have the required continuity of the boundary values. In the Kato-Kuroda theory, $X$ is an abstract space which can be chosen in various ways.

Step 2. Restrict acceptable potentials, $V$, to functions $V: X^{*} \rightarrow X$ or, more generally so that $V\left(H_{0}-E_{0}+1\right)^{-1}$ is bounded from $X$ to itself. In fact, we require this to be a compact operator from $X$ to itself. In the Agmon $L_{\beta}^{2}$ case, for $H_{0}=$ $-\Delta$, one needs that $\left(1+|x|^{2}\right)^{\beta} V(-\Delta+1)^{-1}$ is compact as an operator on $L^{2}$. In 


\section{B. Simon}

particular, if (15.1) holds, we need that $\alpha>2 \beta$, so if $\alpha>1$, we can pick $\beta$ with $1 / 2<\beta<\alpha / 2$. Thus, the following results will solve problems (A) and (B) when $\alpha>1$.

Step 3. For simplicity, we henceforth suppose $E_{0}=0$ and that $E_{0}$ is the only critical point as happens for the Schrödinger case. Under these assumptions, $B(z)=$ $-\left(H_{0}-\kappa^{2}\right)^{-1} V$ for $z=\kappa^{2}$; $\operatorname{Im} \kappa \geq 0, \kappa \neq 0$ is compact operator on $X^{*}$, continuous in $\kappa$ and analytic for $\kappa \in \mathbb{C}_{+}$. By a version of the analytic Fredholm theorem (see [476, Theorem VI.14]), there is a set $\mathcal{E} \subset(0, \infty)$, so that $\mathcal{E}$ is a closed set (i.e. its only limit points are in $\mathcal{E}$ or are 0 or $\infty$ ) of (real) Lebesgue measure 0 and so that if $z \notin \mathcal{E}$, then $(\mathbf{1}-B(z))^{-1}$ exists and is continuous in $z$ there. One proves that $(H-z)^{-1}=(1-B(z))^{-1}\left(H_{0}-z\right)^{-1}$ originally for $\operatorname{Im} z \neq 0$ and then as maps from $X$ to $X^{*}$ for $z \notin \mathcal{E}$.

Step 4. This suffices to get existence and completeness of wave operators. KatoKuroda 350 349] have arguments to get this. In his original announcement, Agmon [3] did not mention scattering. If one can decompose $V=A^{*} B$ so that $A, B: X^{*} \rightarrow$ $L^{2}$ (perhaps after multiplication by $\left(H_{0}+1\right)^{-1 / 2}$ ), then one can show that $A, B$ are locally smooth for both $H$ and $H_{0}$ on $(0, \infty) \backslash \mathcal{E}$ and so by Theorem 14.10, one gets existence and completeness (ideas due to 398, 400). In later publications, Agmon and Hörmander have other ways of proving existence and completeness by exploiting a radiation condition.

Step 5. In general, from this, one gets purely a.c. on $(0, \infty) \backslash \mathcal{E}$ so any singular spectrum on $(0, \infty)$ lies in $\mathcal{E}$.

Step 6. Suppose we show that any $\lambda_{0} \in \mathcal{E}$ is an $L^{2}$ eigenvalue of $H$. Then $\mathcal{E} \cup$ $\{0, \infty\}$ is a countable closed subset of $\mathbb{R}$ which cannot support a singular continuous measure. In this way, one solves problem (B).

Step 7. If $\varphi \in L_{-\beta}^{2}$ and $B\left(\lambda_{0}+i 0\right) \varphi=\varphi, \lambda_{0}=\kappa^{2}$, then

$$
\begin{aligned}
0=\operatorname{Im}\langle V \varphi, \varphi\rangle & =\operatorname{Im}\left\langle V \varphi,\left(H_{0}-\lambda_{0}-i 0\right)^{-1} V \varphi\right\rangle \\
& =\frac{\pi \kappa^{\nu-2}}{2}\left\|T_{\kappa} V \varphi\right\|^{2}
\end{aligned}
$$

so $T_{\kappa} V \varphi=0$. Therefore by (15.21), $Q_{\kappa} V \varphi=B(\kappa) \varphi \in L_{\alpha-\beta-1-\epsilon}^{2}$ for all $\epsilon>0$. For example, if $\alpha>3 / 2$, we can pick $\beta>1 / 2$ but close to it and $\epsilon$ small so that $\alpha-\beta-1-\epsilon \geq 0$. Thus $\varphi \in L^{2}$ and is an eigenfunction. By invoking Step 6 , we see that when $\alpha>3 / 2$, we can solve problem (B). The restriction $\alpha>5 / 4$ in Theorem 15.1 comes from a consideration like this — what is needed to deduce that $\varphi \in L^{2}$.

Step 8. Agmon had the idea of iterating the argument in Step 7! If we know that $\varphi \in L_{\gamma}^{2}$, since $T_{\kappa} V \varphi=0$, we have that $\varphi \in L_{\alpha+\gamma-1-\epsilon}^{2}$, so if $\alpha>1$, we can increase $\gamma$ by an arbitrary amount less than $\alpha-1$. If $\alpha-1>1 / 2 m$ starting in $L_{-\beta}^{2}$ with 
$\beta$ very close to $1 / 2$, we see by iterating $m$ times that $\varphi$ is an $L^{2}$ eigenfunction. In this way, one solves problem (B) for all $\alpha>1$.

Step 9. Once one controls the resolvent, one can obtain eigenfunctions via the Lippmann-Schwinger equation. Knowing that $\mathcal{E}$ is countable shows that the expansion only has a.c. spectrum and point spectrum.

This completes our sketch of the scheme behind the work of Kato-Kuroda and Agmon; see [479, Sec. XIII.8] for more details. After Agmon's argument appeared, various authors realized that the iteration idea in Step 8 could improve their results. In particular, Kuroda [384, 385] was able to extend the proof of Theorem [15.1 to $\alpha>1$. He extended this work to fairly general elliptic operators.

The ideas in the Agmon et al. work have been extended to long-range potentials (where (15.1) holds for suitable $\alpha \in(0,1]$ but we also have $(1+|x|)^{-1-\alpha}$ decay of $\nabla V)$. One needs to use modified wave operators following Dollard [118]. There is a vast literature and we will not try to summarize it - see the books of DerezińskiGérard [113] and Yafaev [663, 668].

The above approach uses the fact that for $L_{\beta}^{2}, \beta>1 / 2$, there is a map restricting $\hat{\varphi}$ to the sphere. One proves this by essentially flattening the sphere. If we replaced $L_{\beta}^{2}$ by $L^{p}$, we cannot restrict to hyperplanes but remarkably, one can sometimes restrict to curved hypersurfaces like the spheres we needed above. The associated bounds are known as the Tomas-Stein Theorem (see [586, Sec. 6.8]). Ionescu-Schlag 258 have developed a theory of scattering and spectral theory under suitable $L^{p}$ conditions on $V$ using the Tomas-Stein bounds.

\section{Scattering and Spectral Theory, IV: Jensen-Kato Theory}

This is the last section on "scattering and spectral" theory although it involves something closer to diffusion than scattering and the connection to spectral theory is weak. Still, since it involves large time behavior of $e^{-i t H}$, it belongs in this set of ideas. In any event, we will discuss a lovely paper of Jensen and Kato 275] involving Schrödinger operators, $H=-\Delta+V$, on $\mathbb{R}^{3}$.

One issue that they discuss is the large time behavior of $e^{-i t H}$ and its rate of decay. At first sight, speaking of decay seems puzzling since for $\varphi \in L^{2}$, we have that $\left\|e^{-i t H} \varphi\right\|_{2}=\|\varphi\|_{2}$ has no decay. But consider the integral kernel when $V=0$ on $\mathbb{R}^{\nu}$

$$
e^{i t \Delta}(x, y)=(4 \pi i t)^{-\nu / 2} e^{i|x-y|^{2} / 4 t}
$$

which shows that

$$
\sup _{x, y}\left|e^{i t \Delta}(x, y)\right|=(4 \pi|t|)^{-\nu / 2}
$$

so

$$
\left\|e^{i t \Delta} \varphi\right\|_{\infty} \leq(4 \pi|t|)^{-\nu / 2}\|\varphi\|_{1}
$$

(16.3) is, in fact, equivalent to (16.2). Since Jensen and Kato use Hilbert space methods, instead of maps from $L^{1}$ to $L^{\infty}$, they consider maps between weighted 
$L^{2}$ spaces, specifically from $L_{s}^{2}$ to $L_{-s}^{2}$, where $L_{s}^{2}$ is given by (15.17). For example, (16.3) immediately implies that $\left\|e^{i t \Delta} \varphi\right\|_{2,-s} \leq C_{\nu, s} t^{-\nu / 2}\|\varphi\|_{2, s}$ so long as $s \geq \nu / 2$.

If $H_{0}=-\Delta$ is replaced by $H=H_{0}+V$, there is a new issue that arises. If $H \varphi=E \varphi$ for $\varphi \in L^{2}$, then $e^{-i t H} \varphi=e^{-i t E} \varphi$ has no decay in any norm. Thus one must only try to prove decay of $e^{-i t H} P_{c}(H)$, where $P_{c}(H)$ ( "c" is for continuous spectrum; if there is no singular continuous spectrum, it is the same as $\left.P_{\mathrm{ac}}\right)$ is the projection onto the orthogonal complement of the eigenvectors. Jensen-Kato do not use $e^{-i t H} P_{c}(H)$ but the equivalent

$$
e^{-i t H}-\sum_{j=1}^{N} e^{-i E_{j} t} P_{j},
$$

where $\left\{E_{j}\right\}_{j=1}^{N}$ are the eigenvalues and $P_{j}$ the projections onto the associated eigenspace $\operatorname{ker}\left(H-E_{j}\right)$.

In the free case, we note that it is easy to see [478, Corollary to Theorem XI.14] that if $0 \notin \operatorname{supp}(\widehat{\varphi})$ for $\varphi \in \mathcal{S}\left(\mathbb{R}^{\nu}\right)$, then $\sup _{|x| \leq R}\left|e^{i t \Delta} \varphi(x)\right|$ is $\mathrm{O}\left(t^{-N}\right)$ for all $N$. That is the diffusive term $t^{-\nu / 2}$ is connected to low energies. A critical realization of Jensen-Kato is that large $t$ asymptotics as maps of $L_{s}^{2}$ to $L_{-s}^{2}$ is connected to the behavior of the resolvent $(H-z)^{-1}$ near $z=0$.

For a while now we return to $\nu=3$, the only case considered by Jensen-Kato. As we will see, $\nu=3$ is perhaps the simplest case with a rich structure. Roughly speaking, Jensen-Kato consider $V^{\prime} s$ obeying

$$
|V(x)| \leq C(1+|x|)^{-\beta} .
$$

They always require $\beta>2$ and often need $\beta>3$ or even larger. In fact, for some of their results, they only need $(1+|x|)^{\beta} V \in L_{\text {unif }}^{3 / 2}$, but for simplicity, we will only quote the following results where the pointwise bound (16.5) holds. Prior to their paper, there was work of Rauch 472] which motivated them. He supposed $|V(x)| \leq$ $C_{1} e^{-C_{2}|x|}$ and instead of $L^{2}$-operator norms of $(1+|x|)^{-s} e^{-i t H} P_{c}(H)(1+|x|)^{-s}$, he considered norms $e^{-\epsilon|x|} e^{-i t H} P_{c}(H) e^{-\epsilon|x|}$. He found for all but a discrete set of $\xi \in \mathbb{R}$, with $H(\xi)=-\Delta+\xi V$, one has $t^{-3 / 2}$ decay for the relevant norms of $e^{-i t H(\xi)}$ and, for a discrete set of $\xi$ 's, $t^{-1 / 2}$ decay. Jensen-Kato extended this result for $L_{s}^{2}$ to $L_{-s}^{2}$ with $s>5 / 2$ and $\beta>3$. Several years earlier, Yafaev [662], in connection with his work on the Efimov effect 659], had studied low energy behavior of the resolvent (but not high energy asymptotics of the unitary group) in the case of a zero energy resonance (case (1) in the language of Jensen-Kato).

It is natural to restrict at least to $\beta>2$ for small energy behavior. The BirmanSchwinger kernel [587, Sec. 7.9], $|V(x)|^{1 / 2} V(y)^{1 / 2} / 4 \pi|x-y|$, is Hilbert-Schmidt if (16.5) holds for $\beta>2$ and in general may not even be a bounded operator if $\beta<2$ (and if $\beta=2$, it can be bounded but not compact). Thus, $\beta>2$ implies that $-\Delta+V$ has only finitely many negative eigenvalues, each of finite multiplicity.

As we have mentioned, the key input for the Jensen-Kato large time results is an analysis of the resolvent, $R(z)=(H-z)^{-1}$ for $z$ near zero. The free resolvent 
$R_{0}(z)=\left(H_{0}-z\right)^{-1}$ has integral kernel

$$
G_{0}(x, y ; z)=\frac{e^{i \kappa|x-y|}}{4 \pi|x-y|},
$$

where $\kappa$ obeys $\kappa^{2}=z$ with $\operatorname{Im}(\kappa)>0$ for $z \in \mathbb{C} \backslash[0, \infty)$ (with obvious limits if $z$ approaches $\mathbb{R}$ from either $\mathbb{C}_{+}$or $\mathbb{C}_{-}$). It is only in dimension 3 (and 1 ) that $G_{0}$ is so simple; in other dimensions, it is a more complicated Bessel function. For $z \in \mathbb{C} \backslash[0, \infty)$, one has that

$$
R(z)=\left(1+R_{0}(z) V\right)^{-1} R_{0}(z) .
$$

Following Agmon and Kuroda (see Sec. [15), Jensen-Kato use the weighted Sobolev spaces, $H^{m, s}\left(\mathbb{R}^{3}\right)$ of those $\varphi$ which obey

$$
\|\varphi\|_{m, s}=\left\|\left(1+|x|^{2}\right)^{s / 2}(1-\Delta)^{m / 2} \varphi\right\|_{2}<\infty .
$$

For example, we can take the completion of $\mathcal{S}\left(\mathbb{R}^{3}\right)$ in this norm or, since $(1+$ $\left.|x|^{2}\right)^{s / 2}(1-\Delta)^{m / 2}$ is a map of tempered distribution to themselves, we can take those tempered distributions for which the quantity in the norm on the right of (16.8) is in $L^{2}$.

Let $K_{0}$ be the operator with integral kernel $(4 \pi|x-y|)^{-1}$, i.e. $G_{0}(x, y ; 0)$. JensenKato prove that if $V$ obeys (16.5) with $\beta>2$, then $K_{0} V$ is a compact operator on $L_{-s}^{2}$ if $1 / 2<s<\beta-1 / 2$, indeed it is compact on $H^{1, s}$. It is also true that extended from $\kappa \in \mathbb{C}_{+}$to $\kappa \in \mathbb{C}_{+} \cup \mathbb{R}, V R_{0}\left(\kappa^{2}\right)$ is Hölder continuous (and compact). While Jensen-Kato do not prove it that way, we note that this follows from the generalized Stein-Weiss inequalities [586, Theorem 6.2.5].

Thus, to understand the small $z$ behavior of $R(z)$, we need to know about $\left(1+K_{0} V\right)^{-1}$. By compactness, this inverse exists if and only if

$$
\left(1+K_{0} V\right) \varphi=0
$$

has no nonzero solutions, $\varphi \in H^{1,-s}$. If $\varphi$ obeys (16.9), it is a distributional solution of $(-\Delta+V) \varphi=0$. Let $\mathcal{M}$ be the set of all solutions of (16.9) in $H^{1,-s}$; Jensen-Kato prove that it is independent of which $s$ is chosen in $(1 / 2, \beta-1 / 2)$. By compactness, $\operatorname{dim} \mathcal{M}<\infty$. It is important to know if $\varphi \in L^{2},(16.9)$ says that

$$
\varphi(x)=-\frac{1}{4 \pi} \int \frac{1}{|x-y|} V(y) \varphi(y) d^{3} y,
$$

so that

$$
\varphi(x)=-\frac{1}{4 \pi|x|} \int V(y) \varphi(y) d^{3} y+o\left(\frac{1}{|x|}\right) .
$$

Thus, if $\int V(y) \varphi(y) d^{3} y \neq 0$, then $\varphi \notin L^{2}$. One can show that if $\int V(y) \varphi(y) d^{3} y=0$, then $\varphi \in L^{2}$. Thus, in $\mathcal{M}$, the set of $L^{2}$ solutions is either all of $\mathcal{M}$ or a space of codimension 1 . If $\mathcal{M}$ has non- $L^{2}$-solutions, we say that there is a zero energy resonance. Jensen-Kato thus consider four cases:

(0) (regular case) $\mathcal{M}=\{0\}$ so $\left(1+K_{0} V\right)^{-1}$ exists. Since $K_{0} V$ is compact, the set of $\xi \in \mathbb{R}$ for which $\xi V$ is not regular is a discrete set. 
(1) (pure resonant case) $\mathcal{M} \neq\{0\}$ but there are no $L^{2}$ functions in $\mathcal{M}$. This implies that $\operatorname{dim} \mathcal{M}=1$.

(2) (pure eigenvalue case) $\mathcal{M} \neq\{0\}$ and $\mathcal{M} \subset L^{2}$. Thus 0 is an eigenvalue but there is no resonance.

(3) (mixed case) $\mathcal{M} \neq\{0\}$ and $\mathcal{M}$ contains both $L^{2}$ and non- $L^{2}$ functions. Then $\operatorname{dim} \mathcal{M} \geq 2$ and the set of $L^{2}$ solutions has codimension 1 .

Later, we will see that in a sense, case (1) is generic among the singular cases. We will see similar qualitative behavior in the three singular cases but the detailed expressions for coefficients depend on the case.

Jensen-Kato start by noting the expansion in $\kappa=\sqrt{z}$ when $V=0$. Given (16.6), we see that

$$
R_{0}\left(\kappa^{2}\right)=\sum_{j=0}^{\infty}(i \kappa)^{j} K_{j},
$$

where $K_{j}$ has the integral kernel

$$
K_{j}(x, y)=|x-y|^{j-1} / 4 \pi j ! .
$$

Then, for $j \geq 1, K_{j}$ is bounded from $H^{-1, s}$ to $H^{1,-s}$ if and only if $s>j+1 / 2$. That means if we fix $s$, we have an asymptotic series only to any order $J<s-1 / 2$. Since $V$ obeying (16.5) maps $L_{-s}^{2}$ to $L_{s}^{2}$ if and only if $s<\beta / 2$, we see that for fixed $\beta$, we can only expect to get an expansion including $\kappa^{j}$ terms if $j<\frac{1}{2}(\beta-1)$. This explains the conditions on $\beta$ in the following theorems. Jensen-Kato prove, with explicit formulae for $B_{j}^{(0)}, j=0,1$, the following.

Theorem 16.1 ([275]). Assume that $V$ is regular at $\kappa=0, \beta>3$ and $s>3 / 2$. Then for explicit operators $B_{0}^{(0)} \neq 0$ and $B_{1}^{(0)}$ from $L_{s}^{2}$ to $L_{-s}^{2}$ as operators between those spaces and $\operatorname{Im} \kappa \geq 0$,

$$
R\left(\kappa^{2}\right)=B_{0}^{(0)}+i \kappa B_{1}^{(0)}+o(\kappa) .
$$

If $\beta>5$ and $s>5 / 2$, then $o(\kappa)$ can be replaced by $O\left(\kappa^{2}\right)$.

They also prove (with explicit formula for $B_{j}^{(k)}$ ) the following.

Theorem 16.2 ([275]). Assume that $V$ is not regular at $\kappa=0, \beta>5$ and $s>5 / 2$. Then for explicit operators $B_{-2}^{(k)}$ and $B_{-1}^{(k)}, k=1,2,3$ from $L_{s}^{2}$ to $L_{-s}^{2}$ as operators between those spaces and $\operatorname{Im} \kappa \geq 0$, one has that

$$
R\left(\kappa^{2}\right)=-\kappa^{-2} B_{-2}^{(k)}-i \kappa^{-1} B_{-1}^{(k)}+O(1)
$$

if the singular point is of type $k$. Moreover, if $k=1, B_{-2}^{(1)}=0, B_{-1}^{(1)} \neq 0$ and if $k=2,3$, then $B_{-2}^{(k)} \neq 0$.

Remarks. (1) The explicit formulae have $B_{j}^{(k)}$ of finite rank for $k=-2,-1$. If $\beta$ and $s$ are large enough, there should be asymptotic series of any prescribed order and the coefficients are all finite rank [433, 276, Proposition 7.1]. 
(2) Rauch 472 says that $B_{-1}^{(k)} \neq 0$ for all $k$ but Jensen-Kato have an explicit example, where $B_{-1}^{(2)}=0$.

(3) Using ideas from Klaus-Simon 361] (discussed further as follows), one can prove not only that regular $V$ 's are generic but among the irregular $V$ 's, type (1) is generic and among those not of type (1), type (3) is generic. For example, one can prove that for any $\beta>5$, if $X_{\beta}=\left\{V\left|\|V\|_{\beta}=\sup _{x}\right|(1+|x|)^{\beta}|V(x)|<\infty\right\}$, then the regular $V$ 's are a dense open set and, in the set, $\widetilde{X}_{\beta}$ of not regular $V$ 's (which is closed and so a complete metric space), the set of type (1) $V^{\prime}$ 's is a dense open set. Klaus-Simon only discuss $V \in C_{0}^{\infty}\left(\mathbb{R}^{3}\right)$ but that is for simplicity and their ideas work in this broader context. These genericity results are not true for spherically symmetric $V$ 's. In that case, the space $\mathcal{M}$, if nonzero, generically has a single angular momentum, $\ell$, and always has a finite number of them. For each $\ell$, the set of $V$ 's with only that $\ell$ is a relatively open subset of the closed subset of spherically symmetric elements of $\widetilde{X}_{\beta}$, so none is generic in the singular $V$ 's. $\ell=0$ is type (1), $\ell \neq 0$ is of type (2). Cases of more than one $\ell$ are of type (3) or (1) depending only on whether one of the $\ell$ values is 0 .

Jensen-Kato also studied low energy asymptotics of the $S$-matrix, and, importantly for the study of asymptotics of $e^{-i t H}$, the low energy behavior of the derivative of the spectral measure

$$
\frac{d}{d \lambda} P_{(-\infty, \lambda)}(H) \equiv P_{H}^{\prime}(\lambda)
$$

A little thought about Stone's formula shows that if $R(z)$ has a limit $R(\lambda+i 0)$ uniformly for $\lambda \in(a, b) \subset \mathbb{R}$, then

$$
P_{H}^{\prime}(\lambda)=\pi^{-1} \operatorname{Im} R(\lambda+i 0),
$$

where, for an operator, $A$, one writes $\operatorname{Im} A=\left(A-A^{*}\right) / 2 i$.

Since $z=\kappa(z)^{2}$ with $\operatorname{Im} \kappa>0$ has that $\kappa(\bar{z})=-\overline{\kappa(z)}$, we see that by (16.17) that if

$$
R\left(\kappa^{2}\right)=\sum_{j=-2}^{J}(i \kappa)^{j} Q_{j}+\mathrm{o}\left(|\kappa|^{J}\right)
$$

then $Q_{j}^{*}=Q_{j}$ and so, with $L=\left[\frac{J-1}{2}\right]$,

$$
P^{\prime}(\lambda)=\pi^{-1} \sum_{\ell=-1}^{L}(-1)^{\ell} \sqrt{\lambda}^{2 \ell+1} Q_{2 \ell+1}+o\left(\sqrt{\lambda}^{J}\right) .
$$

In particular, if (16.14) holds (with an $\mathrm{O}\left(\kappa^{2}\right)$ term), then

$$
P^{\prime}(\lambda)=\pi^{-1} B_{1}^{(0)} \lambda^{1 / 2}+\mathrm{O}(\lambda)
$$

and if (16.15) holds, then

$$
P^{\prime}(\lambda)=\pi^{-1} B_{-1}^{(k)} \lambda^{-1 / 2}+\mathrm{O}(1) .
$$

In this way, Jensen-Kato control $P^{\prime}(\lambda)$ for small $\lambda$. 


\section{B. Simon}

They also find a large $\lambda$ result. They prove that for $k=1,2, \ldots$ and $s>$ $k+1 / 2, \beta>2 k+1$, then as maps from $L_{s}^{2}$ to $L_{-s}^{2}$, one has that

$$
\left(\frac{d}{d \lambda}\right)^{k} P^{\prime}(\lambda)=\mathrm{O}\left(\lambda^{-(k+1) / 2}\right)
$$

as $\lambda \rightarrow \infty$.

With these in hand, they can estimate

$$
e^{-i t H} P_{c}(H)=\int_{0}^{\infty} e^{-i t \lambda} P^{\prime}(\lambda) d \lambda .
$$

The large $\lambda$ contribution as $t \rightarrow \infty$ can be controlled by using the repeated integration by parts and the decay estimates in (16.22) on derivatives of $P^{\prime}(\lambda)$. One sees that the integral on the right side of (16.23) is dominated by the small $\lambda$ contributions. Using the fact that the Fourier transform of $\lambda^{(j-1) / 2} \chi_{(0, \infty)}(\lambda)$ is the distribution $(-i t)^{-(j+1) / 2}$ regularized at $t=0$, one sees the following.

Theorem 16.3 ([275]). Let $V$ obeys (16.5) with $\beta>3, s>5 / 2$. Suppose that $V$ is regular at zero energy. As a map from $L_{s}^{2}$ to $L_{-s}^{2}$, we have that as $t \rightarrow \infty$, (16.4) is asymptotic in norm to

$$
-(4 \pi i)^{-1} B_{1}^{(0)} t^{-3 / 2}+o\left(t^{-3 / 2}\right)
$$

Theorem 16.4 ([275]). Let $V$ obeys (16.5) with $\beta>3, s>5 / 2$. Suppose that $V$ has an exceptional point of type (1) at zero energy. Then, for a suitably normalized solution $\psi \in \mathcal{M}$, we have that as a map from $L_{s}^{2}$ to $L_{-s}^{2}$, as $t \rightarrow \infty$, (16.4) is asymptotic in norm to

$$
(\pi i)^{1 / 2} t^{-1 / 2}\langle\psi, \cdot\rangle \psi+o\left(t^{-1 / 2}\right) .
$$

Remark. $\psi$ is normalized by $\int V(x) \psi(x) d^{3} x=\sqrt{4 \pi}$.

That completes our discussion of the Jensen-Kato paper. One obvious question left open by this work is what happens when $\nu \neq 3$. This was answered for $\nu \geq 5$ by Jensen 273 and for $\nu=4$ by Jensen 274 and Murata 433 (who also had results for more general elliptic operators); see also [9, 10. The case $\nu=2$ with $\int V(x) d^{2} x \neq 0$ was treated by Bollé et al. [59] and the general case by JensenNenciu [276]. For $\nu=1$ with exponentially decaying potentials, the behavior was analyzed by Bollé et al. 60, 61] and, in general, by Jensen-Nenciu [276]. Ito-Jensen 262 discuss Jacobi matrices (discrete $\nu=1$ ).

For $\nu \geq 5$, an important observation is that there are no resonances at zero energy. This is because functions $\varphi \in \mathcal{M}$ obey

$$
\varphi(x)=-c_{\nu} \int|x-y|^{-(\nu-2)} V(y) \varphi(y) d^{\nu} y
$$

and so are $\mathrm{O}\left(|x|^{-(\nu-2)}\right)$ at infinity and thus are in $L^{2}$ if $\nu \geq 5$.

There is a difference between odd $\nu$ and even $\nu$, so we begin with $\nu \geq 5$, odd. In that case, for there to be $t^{-\nu / 2}$ decay for $e^{-i t H_{0}}$ from $L_{s}^{2}$ to $L_{-s}^{2}$, we need that 
$P_{0}^{\prime}(\lambda) \sim \lambda^{-(\nu-2) / 2}$ for small $\lambda$. At first sight, this seems surprising since $R_{0}\left(\kappa^{2}\right)$ has $\mathrm{O}(1)$ terms, so we might guess also $\mathrm{O}(\kappa)$ terms. In fact, only even powers of $\kappa$ occur until $\kappa^{\nu-2}$. This can be seen by analyzing the integral kernel for $G_{0}\left(x, y ; \kappa^{2}\right)$ which is a modified Bessel function of the second kind (see [583, discussion following (6.9.35)]) which is how Jensen [273] does it or by looking at (15.19). (It is an interesting exercise to write $T_{\kappa} \varphi$ in terms of Taylor coefficients of $\widehat{\varphi}$ at $k=0$ and so recover the kernels $K_{j}$ of (16.13) for $j$ odd.)

If 0 is not an eigenvalue of $H$, it is easy to prove that as maps from $L_{s}^{2}$ to $L_{-s}^{2}$, for suitable $s$ and $\beta$, one has an asymptotic series for $R\left(\kappa^{2}\right)$ whose first odd term is $(i \kappa)^{\nu-2}$ and then that $e^{-i t H} P_{c}(H)$ as a map between suitable $L_{r}^{2}$ spaces is $\mathrm{O}\left(t^{-\nu / 2}\right)$. If zero is an eigenvalue and $\beta$ and $s$ are large enough, one can have any of $\mathrm{O}\left(t^{-\frac{1}{2} \nu+2}\right), \mathrm{O}\left(t^{-\frac{1}{2} \nu+1}\right)$ or $\mathrm{O}\left(t^{-\frac{1}{2} \nu}\right)$ and all three possibilities can occur.

Looking at the odd $\nu$ situation, it seems surprising that one can have $\mathrm{O}\left(t^{-m}\right)$ for $m \in \mathbb{Z}$ but it happens when $\nu$ is even for the free case. In fact, if there were an asymptotic series in powers of $\kappa$, the imaginary part cannot have even powers of $\kappa$ as we have seen. The point is that in even dimensions, the Bessel functions have log terms and for $m \in \mathbb{Z}$, we have that $\operatorname{Im}\left[\lambda^{m} \log (-\lambda+i 0)\right]=\pi \lambda^{m}$. Because of this, all the above odd $\nu \geq 5$ results extend to even $\nu \geq 5$.

For $\nu=4$, there can be a resonance and/or bound state as when $\nu=3$, so there are three types of singular points. In the regular case, the leading term is $\mathrm{O}\left(t^{-2}\right)$, but when $\beta$ and $s$ are sufficiently large, the next term is $\mathrm{O}\left(t^{-3} \log (t)\right)$ (unlike $\nu=3$ where the term after $\mathrm{O}\left(t^{-3 / 2}\right)$ is $\left.\mathrm{O}\left(t^{-5 / 2}\right)\right)$. If there is a singular point with only bound states, the leading term is $\mathrm{O}\left(t^{-1}\right)$ but when there are resonances, there is only a bound by $\mathrm{O}(1 / \log t)$.

Jensen-Nenciu [276] analyze $\nu=1,2$ with a new method that also works in general dimension. These dimensions are special in that there is a zero energy resonance for $H_{0}=-\Delta$ - this is especially clear in the coupling constant threshold point of view discussed soon. For $\nu=1$, if $\int_{-\infty}^{\infty}|x||V(x)| d x<\infty$, it is known that every nonzero solution of $-\varphi^{\prime \prime}+V \varphi=0$ is either asymptotic to $a_{ \pm} x+\mathrm{o}(x)$ as $x \rightarrow \pm \infty$ with $a_{ \pm} \neq 0$ or is asymptotic to $b_{ \pm}+\mathrm{o}(1)$ with $b_{ \pm} \neq 0$ (in which case we say that $a_{ \pm}=0$ ). Thus, 0 is never an eigenvalue and is a resonance if and only if there is $\varphi$ with $a_{+}=a_{-}=0$. For suitable $s$ and $\beta$ in the right norm, $e^{-i t H}$ is $\mathrm{O}\left(t^{-3 / 2}\right)$ in the regular case, while in the resonance case, one can have $\mathrm{O}\left(t^{-1 / 2}\right)$ behavior. $\nu=2$ is very much involved. The resonant subspace can be of dimension up to 3 and the small $\kappa$ expansion is jointly in $\kappa$ and $\log (\kappa)$.

Next, we want to mention the connection between resonances and coupling constant behavior. Simon 558 considered $A+\xi B$ for general self-adjoint operators, $A$ and $B$, where $A \geq 0,|B|^{1 / 2}(A+1)^{-1 / 2}$ is compact and $0 \in \sigma_{\text {ess }}(A)$ so that $N(\xi) \equiv \operatorname{dim} \operatorname{ran} P_{(-\infty, 0)}(A+\xi B)<\infty$ for all $\xi \in(0, \Xi)$. Then $N$ is increasing and there is a discrete set $0 \leq \xi_{1} \leq \xi_{2} \leq \cdots$ so that $N(\xi) \geq j \Leftrightarrow \xi>\xi_{j}$. That is, the $\xi_{j}$ are coupling constant thresholds, where, depending on whether you think of $\xi$ as increasing or decreasing, new eigenvalues are born out of 0 or old ones are absorbed. 
Simon proves that

$$
\lim _{\xi \downarrow \xi_{j}}-\frac{E_{j}(\xi)}{\xi-\xi_{j}}
$$

always exists and is nonzero if and only if 0 is an eigenvalue of $A+\xi_{j} B$ (with a more complicated statement if $\xi_{k}=\xi_{j}$ for some $k \neq j$ ).

This links up to the Kato-Jensen work in that the $\xi$ 's, where $\mathcal{M}\left(H_{0}+\xi V\right) \neq\{0\}$ are exactly the coupling constant thresholds. If there are eigenvalues $E_{j}(\xi)$ for $\xi>\xi_{j}$ with $E_{j}\left(\xi_{j}\right)=0$ and $E_{j}(\xi) \leq-c\left(\xi-\xi_{j}\right) ; c>0$, then $H_{0}+\xi_{j} V$ has a zero eigenvalue. If instead $E_{j}=\mathrm{o}\left(\xi-\xi_{j}\right)$, then there is a resonance. For Schrödinger operators, this was explored by Rauch [473] and by Klaus-Simon 361. In particular, Klaus-Simon show for sufficiently large $\beta,-E_{j}(\xi)=\mathrm{O}\left(\left(\xi-\xi_{j}\right)^{2}\right)$ and, in that case, if $V$ has compact support, $E_{j}(\xi)$ is analytic at $\xi=\xi_{j}$. In the bound state case, they prove that $E_{j}(\xi)$ is not analytic at $\xi_{j}$ (as we will discuss as follows, typically, $E_{j}$ has a nonzero imaginary part for $\xi<\xi_{j}$ and real). These ideas also explain why if $\nu=1$ or $\nu=2, H_{0}$ has a resonance at zero energy since it is known 554] that if $V$ obeys 16.5 for $\nu=1,2$ and $\beta>3$ and $\int V(x) d^{\nu} x \leq 0$, then for all $\xi>0, H_{0}+\xi V$ has a bound state.

Simon 568, 569] discusses large time behavior of the $L^{\infty}$ to $L^{\infty}$ norm of $e^{-t H}$ (note $-t$, not $-i t$ ) when there is and when there is not a zero energy resonance.

If there is a zero-energy eigenvalue at a threshold $\xi_{j}$, then it turns into a negative eigenvalue for $\xi>\xi_{j}$. If $\xi<\xi_{j}$, on the basis of the discussion in Sec. 4 in Part 1, one expects that this half-embedded eigenvalue turns into a resonance (in the sense discussed in that section, not the notion earlier in this section). Its imaginary part is not $\mathrm{O}\left(\left(\xi-\xi_{j}\right)^{2}\right)$ as it is in the normal Fermi golden rule situation discussed in Sec. 4; rather, as shown in [277], one typically has that it is $\mathrm{O}\left(\left|\xi-\xi_{j}\right|^{3 / 2}\right)$. For related results, see [115, 116].

Jensen-Kato discussed dispersive decay in terms of $L_{s}^{2}$ spaces but there has been considerable interest in $L^{p}$ estimates, where for $-\Delta+V$ on $L^{2}\left(\mathbb{R}^{\nu}\right)$, one hopes, based on the case $V=0$, that for $1 \leq p \leq 2$,

$$
\left\|e^{-i t H} P_{c}(H) \varphi\right\|_{L^{p^{\prime}}\left(\mathbb{R}^{\nu}\right)} \leq C|t|^{-\nu\left(\frac{1}{p}-\frac{1}{2}\right)}\|\varphi\|_{L^{p}\left(\mathbb{R}^{\nu}\right)}
$$

where $p^{\prime}=p / p-1$ is the dual index to $p$. $L^{p}$ norms are translation invariant making 16.27) much more suitable for use in the theory of nonlinear evolution equations so there is a large literature on such estimates.

The first estimates of the type (16.27) were found by Schonbek [516] in 1979 who considered $\nu=3, p=1$ and $V$ small. The first general result for $\nu \geq 3$ and $V$ so that $H$ has neither an eigenvalue nor resonance at zero energy were in a classic paper of Journé et al. 281. (see also [517]).

An interesting approach to (16.27) is due to Yajima 669 672] who asked about when the wave operators are bounded from $L^{p}$ to $L^{p}$. You might think that this has 
nothing to do with (16.27) but since $\left(\Omega^{ \pm}\right)^{*}$ are then bounded from $L^{p^{\prime}}$ to $L^{p^{\prime}}$ and $e^{-i t H} P_{\mathrm{ac}}(H)=\left(\Omega^{ \pm}\right)^{*} e^{-i t H_{0}}\left(\Omega^{ \pm}\right), L^{p}$ estimates on $\Omega^{ \pm}$and 16.27 for $H_{0}$ imply it for $H$.

There is a considerable literature on $L^{p}$ dispersive estimates when 0 is an eigenvalue of resonance. We refer the reader to 673 . which includes many references.

Finally, we note that 153 and 591 have results on low energy behavior of the resolvent of $-\Delta+V$ when asymptotically $V(x) \sim-c|x|^{-\beta}$ with $c>0$ and $\beta \leq 2$. Both discuss low energy resolvent behavior and 153 also discussed long time asymptotics of $e^{-i t H}$.

\section{The Adiabatic Theorem}

In 1950, Kato published a paper in a physics journal (denoted as based on a presentation in 1948) on the quantum adiabatic theorem. It is his only paper on the subject but has strongly impacted virtually all the huge literature on the subject and related subjects ever since (there are more Google Scholar citations of this paper than of [301]). We will begin by describing his theorem and its proof which introduced what he called adiabatic dynamics and I will call the Kato dynamics. We will see that the Kato dynamics defines a notion of parallel transport on the natural vector bundle over the manifold of all $k$-dimensional subspaces of a Hilbert space, $\mathcal{H}$, and so a connection. This connection is called the Berry connection and its holonomy is the Berry phase (when $k=1$ ). All this Berry stuff was certainly not even hinted at in Kato's work but it is implicit in the framework. Then I will say something about the history before Kato and finally a few brief words about some of the other later developments.

To start, we need a basic result about linear ODEs on Banach spaces.

Proposition 17.1. Let $X$ be a Banach space and $\left\{\mathcal{M}_{t}\right\}_{0 \leq t \leq T}$ a family of norm continuous (in $t$ ) linear maps on $X$.

(a) For each $x_{0} \in X$, there is a function $t \mapsto x\left(t ; x_{0}\right) ; 0 \leq t \leq T$ which is $C^{1}$ in $t$ which is the unique solution of

$$
\frac{d}{d t} x(t)=\mathcal{M}_{t}(x(t)) ; \quad x(0)=x_{0} .
$$

Moreover, for each $t$, the map $W(t): x_{0} \mapsto x\left(t ; x_{0}\right)$ is a bounded linear map on $X$ and $t \mapsto W(t)$ is $C^{1}$ and is the unique solution of (17.1) when the map $\mathcal{M}$ acts on the bounded operators on $\mathcal{L}(X)$ by left operator multiplication by $\mathcal{M}_{t}$ with initial condition that $W(0)=\mathbf{1}$.

(b) Let $\mathcal{H}$ be a (separable, complex) Hilbert space and take either $X=\mathcal{H}$ or $X=\mathcal{L}(\mathcal{H})$ and suppose that

$$
\mathcal{M}_{t}(x)=i A(t) x
$$

where $A(t)$ is a norm continuous map to the bounded self-adjoint operators on $\mathcal{H}$. Then there is a $C^{1}$ family of unitary maps, $U(t)$, with $U(0)=\mathbf{1}$ so the solution of 
(17.1) is

$$
t \mapsto U(t) x_{0}
$$

Remarks. (1) In 17.2), $A(t) x$ is either interpreted as applying $A(t)$ to a vector $x \in \mathcal{H}$ or as left multiplication if $x \in \mathcal{L}(\mathcal{H})$.

(2) The $U(t)$ in (17.3) depend only on $\{A(s)\}_{0 \leq s \leq T}$ (indeed only on $s \leq t$ ) and not on $x_{0}$.

(3) The proof is elementary. For (a), one shows that the differential equation with initial condition (17.1) is equivalent to the integral equation

$$
x(t)=x_{0}+\int_{0}^{t} \mathcal{M}_{s}(x(s)) d s
$$

on $C([0, T] ; X)$, the $X$-valued norm continuous functions on $[0, T]$. One then either uses a contraction mapping theorem (if necessary shrinking $T$ to get a contraction and piecing together unique solutions on several intervals) or else one iterates the integral equation proving an estimate that the $n$th new term in the iteration is bounded by $T^{n}\left[\sup _{0 \leq t \leq T}\left\|M_{t}\right\|\right]^{n} / n$ ! to prove that the iteration converges to a convergent sum.

(4) For (b), one sees that if $U(t)$ solves the equation on $\mathcal{L}(\mathcal{H})$ for $x_{0}=\mathbf{1}$, then $U(t) x_{0}$ solves the equation in general. Moreover, by a simple calculation,

$$
\frac{d}{d t} U^{*}(t) U(t)=0 ; \quad \frac{d}{d t} U(t) U^{*}(t)=i\left[A, U(t) U^{*}(t)\right] .
$$

The first equation and $U(0)=\mathbf{1}$ imply immediately that $U^{*}(t) U(t)=\mathbf{1}$. The second equation with initial condition $U(0) U^{*}(0)=\mathbf{1}$ is clearly solved by $U(t) U^{*}(t)=\mathbf{1}$ so by uniqueness of solutions, we see that $U(t) U^{*}(t)=\mathbf{1}$. Thus $U(t)$ is unitary.

The adiabatic theorem considers a family of time-dependent Hamiltonians, $H(s), 0 \leq s \leq 1$ and imagines changing them slowly, i.e. looking at $H(s / T), 0 \leq$ $s \leq T$ for $T$ very large. Thus, we look for $\tilde{U}_{T}(s)$ solving

$$
\frac{d}{d s} \tilde{U}_{T}(s)=-i H(s / T) \tilde{U}_{T}(s), \quad 0 \leq s \leq T ; \quad \tilde{U}_{T}(0)=\mathbf{1} .
$$

Letting $U_{T}(s)=\tilde{U}_{T}(s T), 0 \leq s \leq 1$, we see that $U_{T}(s), 0 \leq s \leq 1$ solves

$$
\frac{d}{d s} U_{T}(s)=-i T H(s) U_{T}(s), \quad 0 \leq s \leq 1 ; \quad U_{T}(0)=\mathbf{1} .
$$

Here is Kato's adiabatic theorem

Theorem $17.2([\mathbf{3 0 0}])$. Let $H(s)$ be a $C^{2}$ family of bounded self-adjoint operators on a (complex, separable) Hilbert space, $\mathcal{H}$. Suppose there is a $C^{2}$ function, $\lambda(s)$, so that for all $s, \lambda(s)$ is an isolated point in the spectrum of $H(s)$ and so that

$$
\alpha \equiv \inf _{0 \leq s \leq 1} \operatorname{dist}(\lambda(s), \sigma(H(s)) \backslash\{\lambda(s)\})>0 .
$$


Let $P(s)$ be the projection onto the eigenspace for $\lambda(s)$ as an eigenvalue of $H(s)$. Then

$$
\lim _{T \rightarrow \infty}(1-P(s)) U_{T}(s) P(0)=0
$$

uniformly in $s$ in $[0,1]$.

Remarks. (1) Thus if $\varphi_{0} \in \operatorname{ran} P(0)$, this says that when $T$ is large, $U_{T}(s) \varphi_{0}$ is close to lying in $\operatorname{ran} P(s)$. That is as $T \rightarrow \infty$, the solution gets very close to the "curve" $\{\operatorname{ran} P(s)\}_{0 \leq s \leq 1}$.

(2) If there is an eigenvalue of constant multiplicity near $\lambda(0)$ for $s$ small, it follows from $(2.1)$ that $P(s)$ and $\lambda(s)$ are $C^{2}$.

(3) It is easy to see that $\operatorname{dim} \operatorname{ran} P(s)$ is constant. It can even be infinite dimensional.

(4) This result is even interesting if $\operatorname{dim} \operatorname{ran} P(s)$ is 1 and/or $\operatorname{dim} \mathcal{H}<\infty$.

(5) Kato made no explicit assumptions on regularity in $s$ saying "Our proof given below is rather formal and not faultless from the mathematical point of view. Of course it is possible to retain mathematical rigour by detailed argument based on clearly defined assumptions, but it would take us too far into unnecessary complication and obscure the essentials of the problem". It is hard to imagine the Kato of 1960 using such language! In any event, the proof requires that $P(s)$ be $C^{2}$.

(6) We will discuss history more later but Kato notes that his work has two advantages over the earlier work of Born-Fock [65]: (1) They assume complete sets of eigenvectors and do not allow continuous spectrum. (2) They assume that $\lambda(s)$ is simple, i.e. $\operatorname{dim} \operatorname{ran} P(s)=1$ while Kato can handle degenerate eigenvalues.

(7) As we will see, the size estimate for (17.9) is $\mathrm{O}(1 / T)$.

Kato's wonderful realization is that there is an explicit dynamics, $W(s)$ for which (17.9) is exact, i.e.

$$
(1-P(s)) W(s) P(0)=0 .
$$

He not only constructs it but proves the theorem by showing that (this formula only holds in case $\lambda(s) \equiv 0)$

$$
\lim _{T \rightarrow \infty}\left[U_{T}(s)-W(s)\right] P(0)=0 .
$$

The $W(s)$ that Kato constructs is called the adiabatic dynamics. It is sometimes called Kato's adiabatic dynamics. We call it the Kato dynamics. Here is the basic result.

Theorem 17.3 (Kato dynamics [300]). Let $W(s)$ solve

$$
\begin{aligned}
\frac{d}{d s} W(s) & =i A(s) W(s), \quad 0 \leq s \leq 1 ; \quad W(0)=\mathbf{1}, \\
i A(s) & \equiv\left[P^{\prime}(s), P(s)\right] .
\end{aligned}
$$


Then $W(s)$ is unitary and obeys

$$
W(s) P(0) W(s)^{-1}=P(s) .
$$

Proof. That $W(s)$ is unitary follows from Proposition 17.1 Note that since $P(s)^{2}=P(s)$, we have that

$$
P^{\prime}(s)=P^{\prime}(s) P(s)+P(s) P^{\prime}(s) \Rightarrow P(s) P^{\prime}(s) P(s)=0
$$

since the first equation and $P^{2}=P$ imply that $P P^{\prime} P=2 P P^{\prime} P$. Expanding the commutator defining $A(s)$ and using $P P^{\prime} P=0$ yields

$$
\begin{aligned}
& i P(s) A(s)=-P(s) P^{\prime}(s), \\
& i A(s) P(s)=P^{\prime}(s) P(s),
\end{aligned}
$$

so by the first equation in 17.15), we have that

$$
P^{\prime}(s)=i[A(s), P(s)]
$$

By 17.12,

$$
\begin{aligned}
(P(s) W(s))^{\prime} & =\left(P^{\prime}(s)+i P(s) A(s)\right) W(s) \\
& =i A(s) P(s) W(s)
\end{aligned}
$$

by (17.18). Taking adjoints,

$$
\left(W(s)^{-1} P(s)\right)^{\prime}=-i W(s)^{-1} P(s) A(s) .
$$

Since $W(s)^{-1} P(s) W(s)=\left(W(s)^{-1} P(s)\right)(P(s) W(s))$, we see that

$$
\begin{aligned}
\left(W(s)^{-1} P(s) W(s)\right)^{\prime}= & i W(s)^{-1} P(s) A(s) P(s) W(s) \\
& -i W(s)^{-1} P(s) A(s) P(s) W(s)=0 .
\end{aligned}
$$

At $s=0$, this is $P(0)$ so

$$
W(s)^{-1} P(s) W(s)=P(0)
$$

which is equivalent to 17.14).

Proof of Theorem 17.2, By replacing $H(s)$ by $H(s)-\lambda(s) \mathbf{1}$, we can suppose that $\lambda(s) \equiv 0$ (doing this changes some formulae, particularly the critical 17.25 we will address this after the proof). We will prove that

$$
\left\|U_{T}(s)^{*} W(s) P(0)-P(0)\right\|=\mathrm{O}(1 / T) .
$$

Since $U_{T}$ is unitary, this implies that

$$
\left\|W(s) P(0)-U_{T}(s) P(0)\right\|=\mathrm{O}(1 / T) .
$$

Since $(1-P(s)) W(s) P(0)=(1-P(s)) P(s) W(s)=0$, this implies (17.9) with an explicit $\mathrm{O}(1 / T)$ error estimate. 
Thus we define

$$
G(s)=U_{T}^{*}(s) W(s) P(0)
$$

and compute

$$
G^{\prime}(s)=\left(U_{T}^{*}(s)\right)^{\prime} W(s) P(0)+U_{T}^{*}(s) W^{\prime}(s) P(0) .
$$

Applying * to (17.7) implies that

$$
\left(U_{T}^{*}(s)\right)^{\prime}=i T U_{T}^{*}(s) H(s)
$$

so, using (17.14), the first term in (17.27) is

$$
i T U_{T}^{*}(s) H(s) W(s) P(0)=i T U_{T}^{*}(s) H(s) P(s) W(s)=0
$$

since $\lambda(s) \equiv 0 \Rightarrow H(s) P(s)=0$. This is useful because it says that a potential $\mathrm{O}(T)$ term is zero!

Next note that since $P P^{\prime} P=0$, we have that $P A P=0$ and thus

$$
\begin{aligned}
P(s) W^{\prime}(s) P(0) & =i P(s) A(s) W(s) P(0) \\
& =i P(s) A(s) P(s) W(s) \\
& =0 .
\end{aligned}
$$

If now $S(s)$ is the reduced resolvent of $H(s)$ (see (2.8)) $S(s) \equiv(1-P(s)) H(s)^{-1}$, then on account of (17.30), we have that

$$
W^{\prime}(s) P(0)=(1-P(s)) W^{\prime}(s) P(0)=H(s) S(s) W^{\prime}(s) P(0)
$$

so by 17.21,

$$
\begin{aligned}
G^{\prime}(s) & =U_{T}^{*}(s) H(s) S(s) W^{\prime}(s) P(0) \\
& =(i T)^{-1}\left[U_{T}^{*}(s)\right]^{\prime} S(s) W^{\prime}(s) P(0)
\end{aligned}
$$

by (17.28). Thus

$$
G(s)-P(0)=(i T)^{-1} \int_{0}^{s}\left[U_{T}^{*}(w)\right]^{\prime} S(w) W^{\prime}(w) P(0) d w .
$$

As we have seen that $U_{T}^{\prime}$ is $\mathrm{O}(T)$ but we can integrate by parts. Since $U_{T}(w)$ has norm one and $S(w)$ and $W^{\prime}(s)$ are bounded, the boundary terms in the integration by parts are $\mathrm{O}(1 / T)$. Since we assumed that $P(s)$ is $C^{2}$, one has that $S^{\prime}(s)$ and $W^{\prime \prime}(s)$ are bounded so the integrand after integration by parts is bounded and we have proven that $\|G(s)-P(0)\|=\mathrm{O}(1 / T)$, i.e. (17.24) holds.

This completes our discussion of what was in this influential paper of Kato. Kato left at least two important items "on the table". One is the possibility of better estimates than $\mathrm{O}(1 / T)$. We discuss this further as follows.

The other item concerns the fact that (17.25) says a lot more than (17.9) (17.9) says that as $T \rightarrow \infty, U_{T}(s)$ maps $\operatorname{ran} P(0)$ to $\operatorname{ran} P(s)$. (17.25) actually tells you 
what the precise limiting map is! One should note that if $\lambda(s)$ is not identically zero, the proper form of (17.25) is

$$
\left\|U_{T}(s) P(0)-e^{-i T \int_{0}^{s} \lambda(s) d s} W(s) P(0)\right\|=\mathrm{O}(1 / T)
$$

One fancy pants way of describing this is as follows. Fix $k \geq 1$ in $\mathbb{Z}$. Let $\mathcal{M}$ be the manifold of all $k$-dimensional subspaces of some Hilbert space, $\mathcal{H}$. We want $\operatorname{dim}(\mathcal{H}) \geq k$, but it could be finite. Or $\mathcal{M}$ might be a smooth submanifold of the set of all such subspaces. For each $\omega \in \mathcal{M}$, we have the projection $P(\omega)$. There is a natural vector bundle of $k$-dimensional spaces over $\mathcal{M}$, namely, we associate to $\omega \in \mathcal{M}$, the space $\operatorname{ran} P(\omega)$. If $k=1$, we get a complex line bundle.

The Kato dynamics, $W(s)$, tells you how to "parallel transport" a vector $v \in \operatorname{ran} P(\gamma(0))$ along a curve $\gamma(s) ; 0 \leq s \leq 1$ in $\mathcal{M}$. In the language of differential geometry, it defines a connection and such a connection has a holonomy and a curvature. In less fancy terms, consider the case $k=1$. Suppose $\gamma$ is a closed curve. Then $W(1)$ is a unitary map of $\operatorname{ran} P(0)$ to itself, so multiplication by $e^{i \Gamma_{B}(\gamma)}$. Returning to $U_{T}$, it says that the phase change over a closed curve is not what one might naively expect, namely $\exp \left(-i \int_{0}^{T} \lambda(s / T) d s\right)=\exp \left(-i T \int_{0}^{1} \lambda(s) d s\right)$. There is an additional term, $\exp \left(i \Gamma_{B}\right)$. This is the Berry phase discovered by Berry [50] in 1983 (it was discovered in 1956 by Pancharatnam [457] but then forgotten). Simon [573] realized that this was just the holonomy of a natural bundle connection and that, moreover, this bundle and connection is precisely the one whose Chern integers are the TKN ${ }^{2}$ integers of Thouless et al. 616] (as discussed by Avron et al. [27]). Thouless got a recent physics Nobel prize in part for the discovery of the $\mathrm{TKN}^{2}$ integers. The holonomy, i.e. Berry's phase, is an integral of the Kato connection $[P, d P]$. As usual, this line integral over a closed curve is the integral of its differential $[d P, d P]$ over a bounding surface. This quantity is the curvature of the bundle and has come to be called the Berry curvature (even though Berry did not use the differential geometric language). Naively $[d P, d P]$ would seem to be zero but it is shorthand for the two-form

$$
\sum_{i \neq j}\left[\frac{\partial P}{\partial s_{i}}, \frac{\partial P}{\partial s_{j}}\right] d s_{i} \wedge d s_{j} .
$$

This formula of Avron et al. [27] for the Berry curvature is a direct descendant of formulae in Kato's paper, although, of course, he did not consider the questions that lead to Berry's phase.

Now, a short excursion into the history of adiabatic theorems. "Adiabatic" first entered into physics as a term in thermodynamics meaning a process with no heat exchange. In 1916, Ehrenfest [133] discussed the "adiabatic principle" in classical mechanics. The basic example is the realization (earlier than Ehrenfest) that while the energy of a harmonic oscillator is not conserved under time-dependent change of the underlying parameters, the action (energy divided by frequency) is 
fixed in the limit that the parameters are slowly changed (the reader should figure out what Kato's adiabatic theorem says about a harmonic oscillator with slowly varying frequency). See 225] for discussion of applications of the classical adiabatic invariant. Interestingly enough, many adiabatic processes in the thermodynamic sense are quite rapid, so the Ehrenfest use has, at best, a very weak connection to the initial meaning of the term!

Ehrenfest used these ideas by asserting that in old quantum theory, the natural quantum numbers were precisely these adiabatic invariants. Once new quantum mechanics was discovered, Born and Fock [65] in 1928 discussed what they called the quantum adiabatic theorem, essentially Theorem 17.2 for simple eigenvalues with a complete set of (normalizable) eigenfunctions. It was 20 years before Kato found his wonderful extension (and then more than 30 years before Berry made the next breakthrough).

Next, we turn to error estimates. The error on the right side of (17.34) is a sum of two terms after an integration by parts: the boundary term and an integral. For the integral, one can reuse (17.29) as we did to get (17.34) and see that the integral is $\mathrm{O}\left(1 / T^{2}\right)$. The boundary term is $\mathrm{O}(1 / T)$ but the coefficients will vanish if $P(s)-P(0)$ and $P(t)-P(s)$ vanish sufficiently fast as $s \downarrow 0$ and $s \uparrow t$. The natural setup is to take $s \in(-\infty, \infty)$ rather than $[0,1]$ and to require that $H( \pm \infty)=$ $\lim _{s \rightarrow \pm \infty} H(s)$ exist with approach $\mathrm{O}\left(1 /|s|^{k}\right)$ for all $k$. If one does this, one gets an adiabatic theorem with $\mathrm{O}\left(1 / T^{k}\right)$ errors for all $k$. Under suitable analyticity conditions on $H(s)$, one can even prove exponential approach, see [44] for an early paper on this subject and [134, 265, 266, 283, 448] for additional discussion. In particular, Joye-Pfister 283] uses arguments very close to Kato's.

The occurrence of the reduced resolvent, $S$, in Kato's approach suggests that an eigenvalue gap is an important ingredient. Nevertheless, there are results on adiabatic theorems without gaps, see [26, 208, for some special situations and [22] for a very general result. Teufel 611] has an alternate proof for this Avron-Elgart result and he has a book 612] on the subject. Avron et al. 23] and Joye [282] have Banach space versions.

For other approaches to adiabatic evolution, see [267, [217]. For some applications, see [29, 362, 31.

\section{Kato's Ultimate Trotter Product Formula}

We begin this section by describing what is called the Lie product formula. Let $A, B$ be two finite matrices over $\mathbb{C}^{n}$. Fix $T>0$ and for $0 \leq s \leq T$, define

$$
g(s)=e^{s(A+B)}-e^{s A} e^{s B} .
$$

Then $g(0)=g^{\prime}(0)=0$ so, by Taylor's theorem with remainder

$$
\|g(s)\| \leq C s^{2} ; \quad 0 \leq s \leq T
$$


Writing

$$
\begin{aligned}
e^{s(A+B)}-\left[e^{s A / n} e^{s B / n}\right]^{n} & =\left[e^{s(A+B) / n}\right]^{n}-\left[e^{s A / n} e^{s B / n}\right]^{n} \\
& =\sum_{j=1}^{n}\left[e^{s(A+B) / n}\right]^{j-1} g\left(\frac{s}{n}\right)\left[e^{s A / n} e^{s B / n}\right]^{n-j}
\end{aligned}
$$

has norm bounded by $n \exp (s(\|A\|+\|B\|))\left\|g\left(\frac{s}{n}\right)\right\| \rightarrow 0$ by (18.2) Thus, for finite matrices, we have that

$$
e^{s(A+B)}=\lim _{n \rightarrow \infty}\left[e^{s A / n} e^{s B / n}\right]^{n}
$$

This is called the Lie product formula. Although it seems he never wrote it down explicitly, Lie did consider differential equation results on groups close to (18.3). In 1959, Trotter [622] proved a version of the Lie product formula for certain semigroups on Banach spaces.

Theorem 18.1 (Trotter product formula). Let $X$ be a Banach space and $S(t)=e^{-t A}, t>0$ and $T(t)=e^{-t B}, t>0$ two strongly continuous semigroups on $X$ that obey

$$
s-\lim _{t \downarrow 0} S(t)=s-\lim _{t \downarrow 0} T(t)=\mathbf{1} ; \quad\|S(t)\|+\|T(t)\| \leq C e^{D t} .
$$

Suppose that the operator closure of $A+B$ on $D(A) \cap D(B)$ generates a strongly continuous semigroup, $W(t)$ "=" $e^{-t(A+B)}$ obeying (18.4), Then

$$
s-\lim _{n \rightarrow \infty}\left[S\left(\frac{t}{n}\right) T\left(\frac{t}{n}\right)\right]^{n}=W(t) .
$$

Remarks. (1) If $S(t)$ is a semigroup obeying (18.4), then one defines

$$
D(A)=\left\{\varphi \mid \lim _{t \downarrow 0}\left(\frac{\mathbf{1}-S(t)}{t}\right) \varphi \text { exists }\right\}
$$

and sets $A \varphi$ to be the limit. One then writes $S(t)=e^{-t A}$.

(2) If $X$ is a Hilbert space, $S(t)$ is self-adjoint and a contraction, then $S(t)=$ $e^{-t A}$ for a positive (possibly unbounded) self-adjoint operator, $A$. This sets up a $1-1$ correspondence between such semigroups and positive self-adjoint operators.

(3) It is a famous theorem of Stone 587, Sec. 7.3] that when $X$ is a Hilbert space, then $S(t)$ is unitary for all $t$ and strongly continuous at 0 (with $S(0)=\mathbf{1}$ ) if and only if $S(t)=e^{-i t A}$ for a self-adjoint operator $A$.

For a very simple proof when $X$ is a Hilbert space, $A$ and $B$ are self-adjoint and $A+B$ is self-adjoint (rather than only esa) on $D(A) \cap D(B)$, see 476 , Theorem VIII.30]. The proof is due to Nelson 443 and looks like the finite matrix proof plus one use of the uniform boundedness principle.

The limitation that $A+B$ has a closure that is a semigroup generator is quite strong. For example, there are cases where $D(A) \cap D(B)=\{0\}$ but formally $A+B$ makes sense. Remarkably, Kato proved a result that, at least for 
self-adjoint contraction semigroups, always holds. Let $A$ and $B$ be self-adjoint operators and $q_{A}, q_{B}$ their closed quadratic forms as discussed in Example 10.3. Their form sum $q_{C}=q_{A}+q_{B}$ is always a closed form but $V_{q_{C}}$ may not be dense. We will write $C=A \dot{+} B$. We need to define $e^{-t C}$ for $C$ 's which are associated to closed quadratic forms where $V_{q}$ might not be dense. We follow the philosophy discussed in Sec. 10 of Part 1 in the discussion of monotone convergence. If $q$ is a closed quadratic form and $C$ is the self-adjoint operator on $\overline{V_{q}}$ with $V_{q}=D\left(C^{1 / 2}\right)$ and $q(\varphi)=\left\langle C^{1 / 2} \varphi, C^{1 / 2} \varphi\right\rangle$ for $\varphi \in V_{q}$, then we define $e^{-t \dot{C}}$ to be the operator

$$
e^{-t \dot{C}}=e^{-t C} P
$$

where $P$ is the orthogonal projection onto $\overline{V_{q}}$. Here is Kato's result.

Theorem 18.2 (Kato's ultimate Trotter product formula [334]). Let $q_{1}, q_{2}$ be two closed quadratic forms on a Hilbert space, $\mathcal{H}$, with associated semigroups $e^{-t \dot{A}}, e^{-t \dot{B}}$. Let $e^{-t \dot{C}}$ be the semigroup associated to the closed form sum $q_{1}+q_{2}$. Then

$$
s-\lim _{n \rightarrow \infty}\left[e^{-t \dot{A} / n} e^{-t \dot{B} / n}\right]^{n}=e^{-t \dot{C}} .
$$

Remarks. (1) The proof is somewhat technical; we refer the reader to 334 or to [476. Theorem S.21]. The proof relies on a general result of Chernoff [85] (see also [476. Theorem S.19]).

(2) Earlier results on Trotter product formula for form sums include Chernoff 85, 86, 88, Faris 143] and Kato himself 331.

(3) It would be nice to have some kind of result for $e^{-i t \dot{C}}$ but it is unlikely there is one when the approximation is applied to a vector not in $\overline{V_{q}}$. That said, (18.7) holds for all $t \in \mathbb{C}$ with $|\arg (t)|<\pi / 2$ and, as explained by Kato in a Note to his paper 334, by an argument that he got from me, one can extend the result from positive self-adjoint $A, B$, to generators of holomorphic contraction semigroups.

(4) It could be argued with some justice that this paper does not so much belong in Kato's work on NRQM but to his work on linear semigroups. But, as found by Nelson [443. (see also [563]), the Trotter product formula is central to the proof of the Feynman-Kac formula and also to interpreting Feynman integrals for $e^{-i t H}$. Moreover, we saw its appearance in Sec. 9 in Part 1 - see Theorem 9.3.

(5) Kato-Masuda 351 found an extension to nonlinear semigroups. Their paper also has a new result in the linear case, namely instead of $A \dot{+} B$, one can consider $k$ positive, self-adjoint operators, $A_{1}, \ldots, A_{k}$ and their form sum $A_{1} \dot{+} \cdots \dot{+} A_{k}$.

Example 18.3. Let $P$ and $Q$ be two orthogonal projections on a Hilbert space. Define

$$
q_{1}(\varphi)=\left\{\begin{array}{ll}
0 & \text { if } \varphi \in \operatorname{ran} P \\
\infty & \text { if } \varphi \notin \operatorname{ran} P
\end{array},\right.
$$


and similarly for $q_{2}$ and $Q$. Then $e^{-t \dot{A}}=P, e^{-t \dot{B}}=Q$ for all $t$. It is easy to see that the form sum $q_{1}+q_{2}$ has the same structure as (18.8) but with $\operatorname{ran} P$ replaced by $\operatorname{ran} P \cap \operatorname{ran} Q$. If $R$ is the projection onto this intersection, then Kato's result says that

$$
\mathrm{s}-\lim _{n \rightarrow \infty}(P Q)^{n}=R .
$$

It is interesting that this geometrically well-known fact is a special case of Kato's result 18.7).

\section{Regularity of Eigenfunctions and the Kato Cusp Condition}

If one wants to understand the wider impact of Kato's work, a good place to get insight is to look at citations at Google Scholar (https://scholar.google.co.il/ scholar?hl=en\&q=tosio+kato). Of course, the publication with the most references by far is Kato's book 332 with over 20,000 citations. In second place (with over 1700 citations) is the 1957 paper 312 discussed in this section. This may be surprising to some, but it reflects its importance to quantum chemists and atomic physicists.

In this paper, Kato begins by saying that he regards this paper as a continuation of 301. In that earlier paper, he stated "If $V$ is the Coulomb potential as in the case of real atoms, it follows that the eigenfunctions satisfy the wave equation everywhere except at singular points of the potential (they are even analytic since the Coulomb potential is an analytic function). Regarding their behavior at these singular points, we can derive no conclusion from the above theorem. A detailed study shows, however, that they are bounded even at such points". He is interested in the properties of $L^{2}$-eigenfunctions and what he calls generalized eigenfunctions or wave packets by which he means $\psi \in \mathcal{H}$ with $\psi \in \operatorname{ran} E_{\Omega}(H)$, where $H$ is a quantum Hamiltonian, $\Omega=[a, b]$, a bounded interval, and $E_{\Omega}(H)$ is a spectral projection [587, Sec. 5.1]. In fact, we will soon see that $\psi \in \operatorname{ran}\left(e^{-s H}\right)$ for some $s>0$ suffices for some of the results that Kato proved. Kato focused on local regularity of $\psi$ with some global estimates (like on $\|\nabla \psi\|_{\infty}$ ). In particular, he delivered on the boundedness result he claimed in 1951.

There is a huge literature on other aspects of eigenfunctions which we will not discuss except for a few words now. First, there is the issue of exponential decay which we mentioned briefly at the end of Sec. 12, in what follows all we will discuss, in the context of proving pointwise bounds, is how to go from $L^{2}$ exponential decay to pointwise exponential decay. Second, there is the literature on the structure of nodes (i.e. the zero set); see, for example, 674. Finally, there are the issues of continuum eigenfunction expansions and the related theorem that $\sigma(H)$ is the closure of the set of $E$ for which $H \psi=E \psi$ has a polynomially bounded solution; see [571, Corollary C.5.5].

Kato considers two classes of Hamiltonians. The first, which we will call general $H$, acts on $L^{2}\left(\mathbb{R}^{\nu N}\right)$ with $\boldsymbol{x}=\left(x_{1}, \ldots, x_{N}\right) ; x_{j} \in \mathbb{R}^{\nu}$ (Kato only considers the case 
$\nu=3$, but we will discuss the more general case as follows). $H$ then has the form

$$
H=-\Delta+\sum_{j=1}^{N} V_{j}\left(x_{j}\right)+\sum_{1 \leq j<k \leq N} V_{j k}\left(x_{j}-x_{k}\right)
$$

with each $V_{j}, V_{j k} \in L^{p}\left(\mathbb{R}^{\nu}\right)+L^{\infty}\left(\mathbb{R}^{\nu}\right)$, where $p$ is $\nu$-canonical (see just prior to Theorem 7.9) so that $H$ is esa- $\nu$ (see Sec. 7 of Part 1). $-\Delta$ assumes equal masses of the light particle and an infinite mass heavy particle but one easily accommodates general masses using the formalism in Sec. 11$]$

Kato also considered what we will call atomic Hamiltonians

$$
H=-\Delta-\sum_{j=1}^{N} \frac{Z}{\left|x_{j}\right|}+\sum_{1 \leq j<k \leq N} \frac{1}{\left|x_{j}-x_{k}\right|}
$$

on $L^{2}\left(\mathbb{R}^{3 N}\right)$. Kato allows Hughes-Eckart terms, allows $Z$ to be $j$-dependent and allows $\frac{z_{j k}}{\left|x_{j}-x_{k}\right|}$ rather than $\frac{1}{\left|x_{j}-x_{k}\right|}$. All these are easy to accommodate as is the molecular case where $\frac{Z}{\mid x_{j}}$ is replaced by

$$
\sum_{\ell=1}^{L} \frac{Z_{\ell}}{\left|x_{j}-R_{\ell}\right|}
$$

Most of the time, for simplicity of exposition, we will discuss the atomic case.

In the atomic case, we will be especially interested in the set of singularities where some $\left|x_{j}\right|$ or $\left|x_{j}-x_{k}\right|$ vanish, i.e.

$$
\Sigma=\left\{\boldsymbol{x}=\left(x_{1}, \ldots, x_{N}\right)\left|\prod_{j=1}^{N}\right| x_{j}\left|\prod_{1 \leq j<k \leq N}\right| x_{j}-x_{k} \mid=0\right\} .
$$

In 312, Kato proved three main theorems. For the first two, we need a definition. Let $0<\alpha \leq 1$ and $j=0$ or 1 . Then

$$
\begin{gathered}
C^{j, \alpha}=\left\{\psi \mid \psi \text { is } C^{j} \text { and obeys (19.5) }\right\}, \\
\exists C \forall_{x, y|| x-y \mid \leq 1}\left|D^{(j)} \psi(x)-D^{(j)} \psi(y)\right| \leq C|x-y|^{\alpha}
\end{gathered}
$$

( $\alpha=1$ is called Lipschitz; otherwise, we are saying the derivative is Hölder continuous). If the constant $C$ in (19.5) is allowed to depend on a compact $K$ requiring $x, y \in K \subset \mathbb{R}^{\nu}$, we say that $\psi \in C_{\mathrm{loc}}^{j, \alpha}$.

Theorem 19.1 ([312]). Let $\nu=3$ and let $V_{j}, V_{j k} \in L^{\sigma}\left(\mathbb{R}^{3}\right)+L^{\infty}\left(\mathbb{R}^{3}\right)$ for some $\sigma \geq 2$. Let $\psi$ be an eigenfunction or wave packet. Then

(a) For all $\alpha$ with $\alpha \leq 1$ and $\alpha<2-\frac{3}{\sigma}$, we have that

$$
\psi \in C^{0, \alpha} \text {. }
$$

(b) If $\sigma>3$, we have that for all $\alpha<1-\frac{3}{\sigma}$ that

$$
\psi \in C^{1, \alpha} \text {. }
$$


The Coulomb case allows any $\sigma$ with $\sigma<3$ but not $\sigma=3$ so it is borderline for $\psi$ being Lipschitz. Nevertheless, Kato proved the following.

Theorem $19.2([312])$. Let $\nu=3$ and let $H$ be an atomic Hamiltonian. Let $\psi$ be an eigenfunction or wave packet. Then $\psi \in C^{0,1}$ (i.e. is Lipschitz). Indeed $\psi$ is $C^{1}$ on $\mathbb{R}^{3 n} \backslash \Sigma$ with $\nabla \psi \in L^{\infty}$.

Remarks. (1) It is easy to see by the fact that $\Sigma$ is closed of measure zero, that the $C^{1}$ result with bounded derivative implies the $C^{0,1}$ result.

(2) As Kato remarks, in the atomic case, there were no previous positive results on regularity of eigenfunctions if $N \geq 2$ although it was known that certain series expansions did not work.

(3) Since the potentials are real analytic on $\mathbb{R}^{3 N} \backslash \Sigma$, it is known by elliptic regularity [466, 165, 184] that genuine eigenfunctions are real analytic on $\mathbb{R}^{3 N} \backslash \Sigma$. So the point of the theorem is control on $\Sigma$ and the uniformity of the bounds.

Kato's third result concerns the exact behavior at the two particle coincidences. To understand why he states the theorem as he does, consider Hydrogen-like Hamiltonians where the eigenfunctions are exactly known.

Example 19.3. Let $h=-\Delta-\frac{2}{|x|}$ on $L^{2}\left(\mathbb{R}^{3}\right)$. It is known [201] that the unnormalized ground $(1 s)$ state is given by

$$
\varphi_{0}(\boldsymbol{r})=e^{-r} ; \quad r=|\boldsymbol{r}|
$$

obeying $h \varphi_{0}=-\varphi_{0}$. Note that $\varphi_{0}$ is not $C^{1}$ at $\boldsymbol{r}=0$ but has a cusp there, i.e.

$$
\nabla \varphi_{0}(\boldsymbol{r})=-\frac{\boldsymbol{r}}{r} e^{-r}
$$

so that the limit of the derivative ar $r=0$ is directionally dependent.

The $2 p$ state (with $m=0$ ) is given by

$$
\varphi_{1}(\boldsymbol{r})=z e^{-r / 2} ; \quad \boldsymbol{r}=(x, y, z) \in \mathbb{R}^{3}
$$

obeying $h \varphi_{1}=-\frac{1}{4} \varphi_{1}$. Thus

$$
\nabla \varphi_{1}(\boldsymbol{r})=-\frac{1}{2} z \frac{\boldsymbol{r}}{r} e^{-r / 2}+(0,0,1) e^{-r / 2} .
$$

This derivative is continuous at $\boldsymbol{r}=0$ and nonzero at $\boldsymbol{r}=0$. Kato had the realization that by taking a spherical average of $\psi$, one captures (at least in the one electron case) exactly the $s$ states which have cusps. That explains why he took the average in the next theorem.

Theorem 19.4 (Kato Cusp condition [312]). Let $H$ be an atomic Hamiltonian and let $\psi$ be an $L^{2}$ eigenfunction for $H$. Let $\boldsymbol{x}=\left(x_{1}, \ldots, x_{N}\right)$. Define on $(0, \infty) \times$ $\mathbb{R}^{3(N-1)}$

$$
\widetilde{\psi}\left(r, x_{2}, \ldots, x_{N}\right)=\frac{1}{4 \pi} \int_{S^{2}} \psi\left(r \omega, x_{2}, \ldots, x_{N}\right) d \omega
$$


where $d \omega$ is the surface measure on the two-dimensional sphere, so $\tilde{\psi}$ is a spherical average. Then except for $\left(x_{2}, \ldots, x_{N}\right)$ in a set of lower dimension (i.e. less than $3 N-3)$, one has that

$$
\left.\frac{\partial \widetilde{\psi}}{\partial r}\right|_{r=0}=-\frac{Z}{2} \psi\left(0, x_{2}, \ldots, x_{N}\right) .
$$

Remarks. (1) 19.13 is the celebrated Kato cusp condition.

(2) There is a similar result at $x_{j}-x_{k}=0 ;-\frac{Z}{2}$ is replaced by $+\frac{1}{2}$.

(3) In (19.13), the left side means to compute the derivative for $r>0$ (using that $\psi$ is $C^{1}$ there $\Rightarrow \widetilde{\psi}$ is $\left.C^{1}\right)$ and then take $r \downarrow 0$. (19.13) says that $\widetilde{\psi}\left(r, x_{2}, \ldots, x_{N}\right)=$ $-\frac{Z}{2} r \psi\left(0, x_{2}, \ldots, x_{N}\right)+\mathrm{o}(r)$ so that, if $\psi\left(0, x_{2}, \ldots, x_{N}\right) \neq 0, \widetilde{\psi}$ has a cusp as it does for Hydrogen.

(4) Most modern variational calculations for atoms and molecules use basis elements that have the cusp condition, so this theorem is very influential.

Kato's proofs depend on rewriting the time-independent Schrödinger equation as an integral equation and analyzing that equation. This completes what we want to say about Kato's paper itself. We turn to later work, first concerning general Hamiltonians and Theorem 19.1. The most powerful results use path integral methods (pioneered by Herbst-Sloan [232, Carmona [81] and Aizenman-Simon [7]; two comprehensive references are [563, 571]) and are expressed in terms of a class of spaces $K_{\nu}^{(\alpha)} ; \nu=1,2, \ldots ; \alpha \in[0,2)$ defined by (we suppose $\nu \geq 2$ and when $\alpha=0$ that $\nu \geq 3$; we refer the reader to [571] for the other cases) the following.

Definition. $K_{\nu}^{(\alpha)}$ is defined by

(a) for $\alpha \in(0,1) \cup(1,2)$ and $\nu \geq 2$ as those $V$ with

$$
\sup _{x} \int_{|x-y| \leq 1}|x-y|^{-(\nu-2+\alpha)}|V(y)| d y<\infty ;
$$

(b) if $\alpha=0$ or $\alpha=1$ and $\nu \geq 3$ by

$$
\limsup _{r \downarrow 0} \int_{x} \int_{|x-y| \leq r}|x-y|^{-(\nu-2+\alpha)}|V(y)| d y=0 .
$$

Remarks. (1) If $\alpha=0, K_{\nu}^{(0)}=K_{\nu}$ as defined in (9.32).

(2) If $\alpha_{1}>\alpha$, then $K_{\nu}^{\left(\alpha_{1}\right)} \subset K_{\nu}^{(\alpha)}$.

(3) If $p>\nu /(2-\alpha)$, then $L_{\text {unif }}^{p} \subset K_{\nu}^{(\alpha)}$ by Hölder's inequality. In particular $v \in L^{\sigma}\left(\mathbb{R}^{3}\right)+L^{\infty}\left(\mathbb{R}^{3}\right) \Rightarrow v \in K_{3}^{(\alpha)}$ so long as $\alpha<2-3 / \sigma$.

(4) As with $K_{\nu}, v(x) \in K_{\nu}^{(\alpha)}$ for $x \in \mathbb{R}^{\nu}$ implies that $V(x, y) \equiv v(x), x \in \mathbb{R}^{\nu}, y \in$ $\mathbb{R}^{\mu-\nu} \Rightarrow V \in K_{\mu}^{(\alpha)}$. Thus in the context of Theorem 19.1, $V_{j}\left(x_{j}\right)$ and $V_{j k}\left(x_{j}-x_{k}\right)$ on $\mathbb{R}^{3 N}$ will lie in $K_{3 N}^{(\alpha)}$ if the $V$ 's, $\alpha$ and $\sigma$ are as in Remark 2. This means that Theorem 19.1 follows from Theorem 19.6. 
(5) As with $K_{\nu}$, these spaces are special cases of a class of spaces of [509]. In this context, they were introduced by Simon [571].

(6) $K_{\nu, \text { loc }}^{(\alpha)}$ is those $V$ whose restriction to each ball in $\mathbb{R}^{\nu}$ lies in $K_{\nu}^{(\alpha)}$.

One of Kato's realizations is that eigenfunctions are bounded and continuous. In this regard, the following is useful.

Theorem 19.5 (Subsolution estimate). Let $V$ be a function on $\mathbb{R}^{\nu}$ with $V \in$ $K_{\nu}$. Let $\psi \in L_{\text {loc }}^{2}$ solve $(-\Delta+V) \psi=0$ in distributional sense. Then $\psi$ is a continuous function and for any $r>0$, there is $C$ depending only on the $K_{\nu}$-norm of $V_{-} \equiv \max (V(x), 0)$ (and, in particular, not on $\psi$ ) so that

$$
|\psi(x)| \leq C \int_{|x-y| \leq r}|\psi(y)| d y .
$$

Remarks. (1) Such estimates go back to Stampacchia [594 and Trudinger 623] who had stronger hypotheses on $V$. For $V \in K_{\nu}$, Agmon [5] Chap. 5] has an analytic proof and Aizenman-Simon [7] a path integral proof; see also 571].

(2) It is enough to have $V_{-} \in K_{\nu}$ and $V_{+} \equiv V+V_{-} \in K_{\nu, \text { loc }}$.

(3) The name comes from the fact that it is a result proven for positive functions, $u$ with $(-\Delta+V) u \leq 0$ (so subsolutions rather than solutions as in subharmonic rather than harmonic). Kato's inequality shows that if $(-\Delta+V) \psi=0$, then $u=|\psi|$ is a subsolution. In this form, the inequality is intimately connected to Harnack's inequality [7, 571].

Subsolution estimates are important because they say that $\psi \in L^{2} \Rightarrow \psi \in$ $L^{\infty}$ (with, in fact, the function going pointwise to zero at $\infty$ ) and so they give the bounded continuous part of Kato's Theorem 19.1 (for eigenfunctions; for wave packets, see below). They also show that $e^{a r} \psi \in L^{2} \Rightarrow e^{a r} \psi \in L^{\infty}$ and so the $L^{2}$ exponential decay estimates discussed in Theorem 12.7 imply pointwise exponential decay.

The following has Theorem 19.1 as a special case.

Theorem 19.6. Let $0<\alpha<2$. Let $V_{-} \in K_{\nu}^{(\alpha)}, V_{+} \in K_{\nu, \text { loc }}^{(\alpha)}$. Let $f \in L^{2}\left(\mathbb{R}^{\nu}\right)$. Then, for each $t>0, e^{-t H} f$ lies in

(a) $C^{0, \alpha}$ if $\alpha \in(0,1)$,

(b) Is $C^{1}$ and in $C^{0,1}$ if $\alpha=1$,

(c) $C^{1, \alpha-1}$ if $\alpha \in(1,2)$

and the norms only depend on $t$, the $L^{2}$-norm of $f$ and the $K_{\nu}$ norm of $V_{-}$.

Remarks. (1) The proof using functional integration can be found in Simon [571, Theorem B.3.5].

(2) For eigenfunctions, there are subsolution-type estimates for the constants in Hölder estimates; see [571, Theorem C.2.5]. 
(3) To control $\nabla \psi$, one needs $\alpha=1$. The Coulomb potentials in atomic and molecular Hamiltonians are in $K_{3 N}^{(\alpha)}$ for $\alpha \in[0,1)$ but not for $\alpha=1$. Nevertheless, Hoffmann-Ostenhof et al. 2237] have proven for such potentials and $L^{2}$ eigenfunctions, one has that

$$
\sup _{|y-x| \leq R}|\nabla \psi(y)| \leq C \sup _{|y-x| \leq 2 R}|\psi(y)|
$$

for any $x$, where $C$ is a universal constant depending only on $R$ and $H$. This includes and improves Kato's theorem 19.2 one improvement is that exponential decay of $\psi$ implies exponential decay of its first derivatives.

There has been considerable literature dealing with the questions discussed in Kato's Theorems 19.2 and 19.4 a substantial fraction of this literature is by Maria and Thomas Hoffmann-Ostenhof and their collaborators. We want to discuss some of the highlights.

The first result sheds additional light on the behavior near pair singularities. We define

$$
\Sigma_{j}=\left\{x|| x_{j} \mid=0\right\} ; \quad \Sigma_{j k}=\left\{x|| x_{j}-x_{k} \mid=0\right\}, \quad j<k
$$

so $\Sigma=\left(\bigcup_{j=1}^{N} \Sigma_{j}\right) \cup\left(\bigcup_{j<k} \Sigma_{j k}\right)$.

Theorem $19.7(\underline{\mathbf{1 5 2}}])$. Let $x^{(0)} \in \Sigma_{1}, x^{(0)} \notin\left(\bigcup_{j=2}^{N} \Sigma_{j}\right) \cup\left(\bigcup_{j<k} \Sigma_{j k}\right)$. Let $\psi$ be an $L^{2}$ eigenfunction of $H$. Then there are two functions, $\varphi_{1}$ and $\varphi_{2}$, defined and analytic in a neighborhood, $Q$, of $x^{(0)} \in \mathbb{R}^{3 N}$, so that in $Q$,

$$
\psi(x)=\varphi_{1}(x)+\left|x_{1}\right| \varphi_{2}(x) .
$$

Remarks. (1) Near $x^{(0)}$,

$$
\psi(x)=\varphi_{1}\left(x^{(0)}\right)+\left|x_{1}\right| \varphi_{2}\left(x^{(0)}\right)+\nabla \varphi_{1}\left(x^{(0)}\right) \cdot\left(x-x^{(0)}\right)+\mathrm{O}\left(\left(x-x^{(0)}\right)^{2}\right)
$$

clearly showing the cusp.

(2) Similar results hold for each $\Sigma_{j}$ and each $\Sigma_{j k}$.

(3) For a proof, see [152]. They were motivated by earlier work of Hill [235].

(4) This shows a cusp, but supplements rather than proves the Kato cusp equality 19.13. Indeed, that equality implies that $\varphi_{2}\left(x^{(0)}\right)=-\frac{Z}{2} \varphi_{1}\left(x^{(0)}\right)$.

The cusp condition only holds at simple singular points where only a single pair among $\left\{0, x_{1}, \ldots, x_{N}\right\}$ coincides (in the atomic case). In 1954, Fock 148] (the same Fock of Born-Fock 26 years earlier and the Hartree-Fock approximation 24 years earlier and of Fock space 22 years earlier!) gave arguments that there are $\left\langle x_{j}, x_{k}\right\rangle \log \left(\left|x_{j}\right|^{2}+\left|x_{k}\right|^{2}\right)$ terms at points where both $\left|x_{j}\right|$ and $\left|x_{k}\right|$ go to zero. These are called Fock terms.

The following includes and improves the Kato cusp condition, Theorem 19.4. 
Theorem $19.8([151])$. On $\mathbb{R}^{3 N}$, let

$$
\begin{aligned}
& F_{2}(x)=-\frac{Z}{2} \sum_{j=1}^{N}\left|x_{j}\right|+\frac{1}{4} \sum_{1 \leq j<k \leq N}\left|x_{j}-x_{k}\right|, \\
& F_{3}(x)=\frac{2-\pi}{12 \pi} \sum_{1 \leq j<k \leq N}\left\langle x_{j}, x_{k}\right\rangle \log \left(\left|x_{j}\right|^{2}+\left|x_{k}\right|^{2}\right) .
\end{aligned}
$$

For any $\varphi$, write

$$
\psi=e^{F_{2}+F_{3}} \varphi
$$

Then, if $\psi$ solves $H \psi=E \psi$ on a bounded set, $\Omega$, we have that

$$
\varphi \in C^{1,1}
$$

Remarks. (1) Writing $\psi$ in the form $e^{F} \varphi$ is often called a Jastrow trial function after Jastrow 268] who had the idea of modifying Slater determinants, $\varphi$ by multiplying by $e^{F}$ with $F$ a simple rational function of the $\left|x_{j}\right|$ and $\left|x_{j}-x_{k}\right|$.

(2) The weaker result where $F_{3}$ is not included and $\varphi \in C^{1, \alpha}$ was proven earlier by Hoffmann-Ostenhof et al. [237. The above theorem is from Fournais et al. [151] where the reader can find a proof that depends on looking at the PDE that $\varphi$ obeys and standard elliptic estimates. All depend on noting that

$$
\Delta F_{2}=V
$$

(3) The reader may be puzzled by $-\frac{Z}{2}$ but $\frac{1}{4}$ rather than $\frac{1}{2}$ (since the effective $Z$ for the $j k$ pair is +1$)$. But $\nabla F_{2}$ has only one $\nabla_{j}$ acting non-trivially on $\left|x_{j}\right|$ but both $\nabla_{j}$ and $\nabla_{k}$ act non-trivially on $\left|x_{j}-x_{k}\right|$ turning the $\frac{1}{4}$ into a $\frac{1}{2}$ which is also why we get (19.24).

(4) [237] noted that their result implies that

$$
\nabla \psi-\psi \nabla F_{2} \in C^{1, \alpha}, \quad \alpha \in(0,1)
$$

while [151] note that their results imply that

$$
\nabla \psi-\psi \nabla\left(F_{2}+F_{3}\right) \in C^{1,1} .
$$

The first is a strong form of the Kato cusp condition (which follows from the continuity of $\boldsymbol{\nabla} \psi-\psi \nabla F_{2}$ ) and unlike Kato, they prove results at multiple coincidences. The second result implies that second derivatives of $\psi$ are bounded at simple coincidences and have a logarithmic blow up at points where $\left|x_{j}\right|$ and $\left|x_{k}\right|$ go to zero.

(5) The obvious extensions hold for molecular Hamiltonians.

(6) A interesting alternate approach to understanding the Kato cusp conditions in terms of singularities at corners is found in [11].

That completes what we want to say about regularity of eigenfunctions; we end this section with a few remarks on the closely related subject of regularity of the 
one electron density defined by

$$
\rho_{\psi}(x)=N \int\left|\psi\left(x, \mathbf{x}_{2}, \ldots, x_{N}\right)\right|^{2} d^{3} x_{2} \cdots d^{3} x_{n}
$$

(this is the formula if $\psi$ is symmetric or antisymmetric; otherwise the " $N$ " in front is replaced by summing against putting $x$ in each of the $N$ slots). It measures the electron density.

Theorem 19.9 ([150]). For any atomic or molecular eigenfunction, the density, $\rho_{\psi}$ is real analytic away from the nuclei $\left(x=0\right.$ in the atomic case and $x=R_{j}, j=$ $1, \ldots, K$ in the molecular case).

This was proven in [150]. Earlier, the same authors had proven that $\rho_{\psi}$ is $C^{\infty}$ [149. Jecko 271 has an alternate proof of Theorem 19.9.

\section{Two Conjectures}

I thought it would be appropriate to end this paper with two open questions in the areas that interested Kato. One dates from 1971 when Kato was still active and the other from 2000, the year after he died.

Conjecture 20.1 (Jörgens' Conjecture). Let $\Omega \subset \mathbb{R}^{\nu}$ be open. Let $V \in L_{\text {loc }}^{2}(\Omega)$, so that $-\Delta+V$ is bounded from below and esa on $C_{0}^{\infty}(\Omega)$. Suppose that $V_{1} \geq V$ is also in $L_{\mathrm{loc}}^{2}(\Omega)$. Then $-\Delta+V_{1}$ is also esa on $C_{0}^{\infty}(\Omega)$.

This result would be interesting even for $\Omega=\mathbb{R}^{\nu}$, where, of course when $V \equiv 0$, this is just the famous result of Kato in Sec. 9 of Part 1 . The case where $\Omega=\mathbb{R}^{\nu}$; $\nu \geq 5$ and $V(x)=-\nu(\nu-4)|x|^{-2}$ (results of Kalf-Walter and Simon) is mentioned in Sec. 9 .

In the early 1970s, there were a set of almost annual meetings at Oberwolfach on spectral and scattering theory and frequent PDE meetings. They were quite important. For example, Agmon announced his result Theorem 15.2 in 1970 [3] but only published the full paper [4] in 1975. In between, the standard source for his work were personal notes some people took of a series of lectures that he gave at one of these Oberwolfach meetings. In connection with the 1971 PDE conference (organized by Haack, Heinz and Hellwig), Konrad Jörgens (1926-1974), who died tragically of a brain tumor less than three years later, made the above conjecture. At the conference, Kalf discussed his work with Walter 289 mentioned in Sec. 9.

Here is the story that Kalf told me:

Before the talks of the conference started Hellwig introduced me to Jörgens and Weidmann (then Jörgens's assistant) and proudly mentioned the result Walter and I had proved. Jörgens's immediate reaction was, "This is false, because the Laplacian is not e.s.a. on $\mathbb{R}^{n} \backslash\{0\} "$. Weidmann interfered with the remark that this depended on the dimension. Jörgens thought for a moment, and then he said "The result is trivial because an e.s.a. operator remains e.s.a. when the potential is increased". Fortunately, I had 
the presence of mind to say that there are examples where $L P C$ and $L C C$ alternate when a parameter is increased. Jörgens was astonished to hear that. After dinner I showed him the corresponding paper by Sears. After a while he said "This is a case where the operator is not bounded from below; it cannot happen for semibounded operators".

Note that Simon's and Kato's papers discussed in the historical part of Sec. 9 were both preprinted in early 1972 after this conjecture, so the original conjecture was made for a local Stummel space rather than $L_{\text {loc }}^{2}$ but eventually, it was updated to $L_{\text {loc }}^{2}$.

In one dimension, this is related to a result of Kurss [387] who proved the result for continuous $V$ although his argument does not need continuity (essentially it follows from a simple comparison argument for positive solutions and limit pointlimit circle methods). In 1966, in [596, Stetkaer-Hansen extended Theorem 8.6 to the case where $V$ is locally Stummel. Since, if $\nu \leq 3, L_{\mathrm{loc}}^{2}$ is the same as locally Stummel, this implies Jörgen's conjecture for $\Omega=\emptyset$ and these $\nu$ (indeed without the need of a comparison potential!).

Many people, especially in the various German groups studying Schrödinger operators worked hard on this problem. In 1980, Cycon [96] proved a result when there was an additional technical condition on $-\Delta+V$. He remarked that given the failure to find a proof, some researchers began to suspect that it might be false.

Conjecture 20.2 (Simon's Conjecture). Let $V$ be a measurable function on $\mathbb{R}^{\nu}, \nu \geq 2$ obeying

$$
\int|x|^{-\nu+1}|V(x)|^{2} d^{\nu} x<\infty .
$$

Then $-\Delta+V$ has a.c. spectrum of infinite multiplicity on $[0, \infty)$.

This was made by Simon [580]. While not explicit, there is a presumption that $-\Delta+V$ is esa- $\nu$. If $V$ obeys (16.5), one needs $\beta>1 / 2$. It would be interesting to prove the conjecture for all $V$ 's obeying (16.5) for any fixed $\beta \in\left(1, \frac{1}{2}\right)$.

Here is some background on the conjecture. Kato-Kuroda-Agmon studied $V$ 's obeying (16.5) for any $\beta>1$ and found (much more than) $\sigma_{\mathrm{ac}}(-\Delta+V)=[0, \infty)$. As noted in Sec. 14. when $\nu=1$, if it is known that for any $\beta<1 / 2$, there are $V$ 's with no a.c. spectrum; in fact, in a sense, this is generic. In the mid-1990s, I realized that determining what happened when $1>\beta>1 / 2$ was a natural problem and alerted my graduate student advisees to this fact. Kiselev [359] proved that when $\nu=1$ and $\beta>3 / 4$, one could prove that $\sigma_{\mathrm{ac}}(-\Delta+V)=[0, \infty)$. (It was eventually realized that this regime differed from $\beta>1$ in that one could also have singular continuous spectrum mixed in). This was then pushed, again when $\nu=1$ to $\beta>1 / 2$ by Christ-Kiselev [90] and Remling [489]. It seemed natural that the precise borderline was $V \in L^{2}$ and in 1999, Deift and Killip (then my PhD. student) 105. proved the following. 
Theorem 20.3 ([105]). Let $V \in L^{2}(\mathbb{R}, d x)$. Then $H=-\frac{d^{2}}{d x^{2}}+V$ on $L^{2}$ has a.c. spectrum $[0, \infty)$ with multiplicity 2 .

If $V(\mathbf{x})=V(|x|)$ is spherically symmetric on $\mathbb{R}^{\nu}$, then 20.1) $\Rightarrow$ $\int_{0}^{\infty}|V(r)|^{2} d r<\infty$, so the Deift-Killip result implies and is essentially equivalent to Conjecture 20.2 for spherically symmetric $V$.

Several people have worked quite hard on this conjecture without success (although sometimes they found weaker results that they published). The reader trying to understand the Deift-Killip result should also consult Killip-Simon 355,356 .

\section{Appendix A. Kato's Proof of his $x^{-1}$ Inequality}

Kato [332, Remark V.5.12] states, without a full proof, that for each $\varphi \in C_{0}^{\infty}\left(\mathbb{R}^{3}\right)$, one has that

$$
\int|x|^{-1}|\varphi(x)|^{2} d^{3} x \leq \frac{\pi}{2} \int|k||\widehat{\varphi}(k)|^{2} d^{3} k .
$$

He does say that this is equivalent to bounding the integral operator (A.5), but he does not explain how to go further. When Hubert visited Kato in Berkeley in 1975, he asked Kato for the proof. Hubert shared what Kato showed him and gave me permission to include it here. Recall that, as we explained after (10.30), this is a special case of a result of Herbst getting the optimal constant for all these scale-invariant inequalities.

Lemma A.1. Let $C$ be an integral operator on $L^{2}(X, d \mu)$ with integral kernel

$$
C(x, y)=A(x, y) B(x, y) \text {. }
$$

Suppose that

$$
\sup _{y} \int|A(x, y)|^{2} d \mu(x)=M_{1}^{2} ; \quad \sup _{x} \int|B(x, y)|^{2} d \mu(y)=M_{2}^{2} .
$$

Then

$$
\|C\| \leq M_{1} M_{2} .
$$

Proof. Let $\varphi, \psi \in L^{2}(M, d \mu)$. Then

$$
\begin{aligned}
|\langle\varphi, C \psi\rangle|= & \left|\int A(x, y) B(x, y) \overline{\varphi(x)} \psi(y) d \mu(x) d \mu(y)\right| \\
\leq & \left(\int|A(x, y)|^{2}|\psi(y)|^{2} d \mu(x) d \mu(y)\right)^{1 / 2} \\
& \times\left(\int|B(x, y)|^{2}|\varphi(y)|^{2} d \mu(x) d \mu(y)\right)^{1 / 2}
\end{aligned}
$$

by the Schwarz inequality. By (A.3), the first integral (integrating first over $x$ ) is bounded by $M_{1}^{2}\|\psi\|^{2}$, so $|\langle\varphi, C \psi \mid\rangle| \leq M_{1} M_{2}\|\varphi\|\|\psi\|$. 


\section{B. Simon}

Next, we include the part that was in Kato's book. A.1 is equivalent to $\left\||p|^{-1 / 2}|x|^{-1}|p|^{-1 / 2}\right\| \leq \pi / 2$, where $|p|$ is the operator $\widehat{|p| \varphi}(p)=|p| \hat{\varphi}(p)$. In " $p$-space", $x^{-1}$ is convolution with the function $(2 \pi)^{-3 / 2} \widehat{x^{-1}}$ (see [583, $\left.(6.2 .45)\right]$ ) and, by [583, Theorem 6.8.1], $\widehat{x^{-1}}(k)=\sqrt{\frac{2}{\pi}}|k|^{-2}$. Thus A.1 is equivalent to a bound on an integral operator

$$
\|C\| \leq \frac{\pi}{2} ; \quad C(k, p)=\frac{1}{2 \pi^{2}} \frac{1}{k^{1 / 2}} \frac{1}{|\boldsymbol{k}-\boldsymbol{p}|^{2}} \frac{1}{p^{1 / 2}} .
$$

We can write $2 \pi^{2} C$ in the form of (A.2), where

$$
A(k, p)=\frac{p^{1 / 2}}{k|\boldsymbol{k}-\boldsymbol{p}|} ; \quad B(k, p)=\frac{k^{1 / 2}}{p|\boldsymbol{k}-\boldsymbol{p}|} .
$$

This factorization is not what one might guess but has the naive expectation multiplied/divided by $(k / p)^{1 / 2}$ (without doing this the integral in (A.7) would diverge). Frank pointed out that a similar idea was used in [213, Sec. 9.3] for not unrelated (but one-dimensional) integral operators. It might have motivated Kato. By the Lemma, we need to compute

$$
\begin{aligned}
\int \frac{p}{k^{2}|\boldsymbol{k}-\boldsymbol{p}|^{2}} d^{3} k & =\int_{0}^{\infty} 2 \pi p\left[\int_{-1}^{1} \frac{d \omega}{k^{2}+p^{2}-2 k p \omega}\right] d k \\
& =2 \pi \int_{0}^{\infty} \frac{1}{k} \log \left[\frac{k+p}{|k-p|}\right] d k
\end{aligned}
$$

where A.7) comes from shifting to polar coordinates with $d^{3} k=\left(k^{2} d k\right) d \varphi d(\cos \theta)$ and $\omega=\cos \theta$. The inner integral gives $\frac{1}{2 p k} \log \frac{(k+p)^{2}}{(k-p)^{2}}$ yielding (A.8).

Using

$$
\int_{0}^{a} \frac{1}{x} \log \left(\frac{a+x}{a-x}\right) d x=\int_{a}^{\infty} \frac{1}{x} \log \left(\frac{x+a}{x-a}\right) d x=\frac{\pi^{2}}{4}
$$

(we defer this calculation) and Lemma A.1 we see that

$$
\|C\| \leq \frac{1}{2 \pi^{2}}(2 \pi) 2 \frac{\pi^{2}}{4}=\frac{\pi}{2}
$$

proving (A.1).

By scaling and changing $x$ to $1 / x$, one sees that the integrals in (A.9) are equal and $a$ independent, so we can take $a=1$ in the first integral. Using first $u=\frac{1+x}{1-x}$ and then $y=\log u$, one sees that

$$
\begin{aligned}
\int_{0}^{1} \frac{1}{x} \log \left(\frac{1+x}{1-x}\right) d x & =2 \int_{1}^{\infty} \frac{1}{u^{2}-1} \log u d u \\
& =2 \sum_{n=1}^{\infty} \int_{1}^{\infty} u^{-2 n} \log u d u
\end{aligned}
$$




$$
\begin{aligned}
& =2 \sum_{n=1}^{\infty} \int_{0}^{\infty} y e^{-(2 n-1) y} d y \\
& =2 \sum_{n=1}^{\infty} \frac{1}{(2 n-1)^{2}}=2\left[\frac{\pi^{2}}{8}\right]
\end{aligned}
$$

since if $Q=\sum_{n=1}^{\infty} 1 / n^{2}$, then $Q=\frac{1}{4} Q+\sum_{n=1}^{\infty}(2 n-1)^{-2}$ so that

$$
Q=\frac{\pi^{2}}{6} \Rightarrow \sum_{n=1}^{\infty} \frac{1}{(2 n-1)^{2}}=\frac{3 Q}{4}=\frac{\pi^{2}}{8}
$$

by the Euler sum. Alternatively (and this is a remark I got from Martin Klaus), one can evaluate the right side, $I$, of (A.11) by the method of contour integrals. First, using that the integral is unchanged by $u \rightarrow u^{-1}$ coordinate changes, note that $I$ is $\int_{0}^{\infty}\left(u^{2}-1\right)^{-1} \log u d u$. This integral is unchanged under rotating the contour by $90^{\circ}$. Since, for $y>0$, we have that $\log (i y)=\log (y)+i \pi / 2$, and, under $u=i y$, we have that $d u /\left(u^{2}-1\right)=-i d y /\left(1+y^{2}\right)$, we see that

$$
I=-i \int_{0}^{\infty} \frac{\log (y)}{y^{2}+1} d y+\frac{\pi}{2} \int_{0}^{\infty} \frac{d y}{y^{2}+1}=\frac{\pi}{2} \frac{\pi}{2}=\frac{\pi^{2}}{4} .
$$

The first integral is 0 by $y \mapsto y^{-1}$.

It might be surprising that (A.1 has equality in the norm since the proof has some inequalities. But the integrals in A.3 are independent of the variable one is taking a sup over so the only inequality is the Schwarz inequality. It is believable that one can come close to saturating that.

Having completed our exposition of Kato's proof, we note that one standard proof (see e.g. [477, p. 169]) of the classical Hardy's inequality in $\mathbb{R}^{3}$ uses

$$
\|\nabla \varphi\|_{2}^{2}-\frac{1}{4}\left\|r^{-1} \varphi\right\|_{2}^{2}=\left\|r^{-1 / 2} \nabla\left(r^{1 / 2} \varphi\right)\right\|_{2}^{2}
$$

Frank et al. 157 have found an analogous formula that proves (A.1) (they also do this for other fractional powers)

$$
\left\langle\psi,\left(\sqrt{-\Delta}-\frac{2}{\pi|x|}\right) \psi\right\rangle=\frac{1}{2 \pi^{2}} \iint_{\mathbb{R}^{3} \times \mathbb{R}^{3}} \frac{|| x|\psi(x)-| y|\psi(y)|^{2}}{|x-y|^{4}} \frac{d x}{|x|} \frac{d y}{|y|} .
$$

This proves strict positivity for any function $\psi$ and, by taking $\psi(x)$ to be $|x|^{-1}$ cutoff near the origin and near infinity, one sees that the constant in A.1 is optimal.

\section{Acknowledgments}

This research was supported in part by NSF Grants DMS-1265592 and DMS1665526 and in part by Israeli BSF Grant No. 2014337. 


\section{References}

[1] 1980 Wiener and Steele Prizes Awarded, Notices Amer. Math. Soc. 27 (1980) 528533.

[2] S. Agmon, Lower bounds for solutions of Schrödinger equations, J. Anal. Math. 23 (1970) 1-25.

[3] S. Agmon, Spectral properties of Schrödinger operators, in Actes du Congrès International des Mathématiciens (Nice, 1970), Tome 2, Gauthier-Villars, Paris (1971), pp. 679-683.

[4] S. Agmon, Spectral properties of Schrödinger operators and scattering theory, Ann. Sc. Norm. Super. Pisa Cl. Sci. 2 (1975) 151-218.

[5] S. Agmon, Lectures on Exponential Decay of Solutions of Second-Order Elliptic Equations: Bounds on Eigenfunctions of $\mathrm{N}$-Body Schrödinger Operators (Princeton University Press, Princeton, 1982).

[6] J. Aguilar and J. M. Combes, A class of analytic perturbations for one-body Schrödinger Hamiltonians, Comm. Math. Phys. 22 (1971) 269-279.

[7] M. Aizenman and B. Simon, Brownian motion and Harnack's inequality for Schrödinger operators, Comm. Pure Appl. Math. 35 (1982) 209-273.

[8] S. Albeverio, On bound states in the continuum of $N$-body systems and the Virial theorem, Ann. Phys. 71 (1972) 167-276.

[9] S. Albeverio, F. Gesztesy and R. Høegh-Krohn, The low energy expansion in nonrelativistic scattering theory, Ann. Inst. H. Poincaré A 37 (1982) 1-28.

[10] S. Albeverio, D. Bollé, F. Gesztesy, R. Høegh-Krohn and L. Streit, Low-energy parameters in nonrelativistic scattering theory, Ann. Phys. 148 (1983) 308326.

[11] B. Ammann, C. Carvalho and V. Nistor, Regularity for eigenfunctions of Schrödinger operators, Lett. Math. Phys. 101 (2012) 49-84.

[12] W. Amrein, A. Boutet de Monvel and V. Georgescu, $C_{0}-$ Groups, Commutator Methods and Spectral Theory of N-Body Hamiltonians (Birkhäuser, Basel, 1996).

[13] W. Amrein and K. Sinha, On pairs of projections in a Hilbert space, Linear Algebra Appl. 208/209 (1994) 425-435.

[14] T. Ando and K. Nishio, Positive selfadjoint extensions of positive symmetric operators, Tohoku Math. J. 22 (1970) 65-75.

[15] M. Arai, On essential self-adjointness of Dirac operators, RIMS Kokyuroku, Kyoto Univ. 242 (1975) 10-21.

[16] M. Arai, On essential selfadjointness, distinguished selfadjoint extension and essential spectrum of dirac operators with matrix valued potentials, Publ. RIMS, Kyoto Univ. 19 (1983) 33-57.

[17] N. Aronszajn, A unique continuation theorem for solutions of elliptic partial differential equations or inequalities of second order, J. Math. Pures Appl. 36 (1957) $235-249$.

[18] N. Aronszajn, On a problem of Weyl in the theory of singular Sturm-Liouville equations, Amer. J. Math. 79 (1957) 597-610.

[19] F. Atkinson and W. Everitt, Bounds for the point spectrum for a Sturm-Liouville equation, Proc. Roy. Soc. Edinburgh Sect. A 80 (1978) 57-66.

[20] J. Avron, Bender-Wu formulas for the Zeeman effect in hydrogen, Ann. Phys. 131 (1981) 73-94.

[21] J. Avron, From mathematical physics to analysis: A walk in Barry Simon's mathematical garden, II, Notices Amer. Math. Soc. 63 (2016) 878-889.

[22] J. Avron and A. Elgart, Adiabatic theorem without a gap condition, Commun. Math. Phys. 203 (1999) 445-463. 
[23] J. Avron, M. Fraas, G. M. Graf and P. Grech, Adiabatic theorems for generators of contracting evolutions, Comm. Math. Phys. 314 (2012) 163-191.

[24] J. Avron and I. Herbst, Spectral and scattering theory of Schrödinger operators related to the Stark effect, Comm. Math. Phys. 52 (1977) 239-254.

[25] J. Avron, I. Herbst and B. Simon, Schrödinger operators with magnetic fields, General interactions, Duke Math. J. 45 (1978) 847-883; Separation of center of mass in homogeneous magnetic fields, Ann. Phys. 114 (1978) 431-451; Atoms in homogeneous magnetic field, Comm. Math. Phys. 79 (1981) 529-572; Strongly bound states of hydrogen in intense magnetic field, Phys. Rev. A 20 (1979) 2287-2296.

[26] J. E. Avron, J. S. Howland and B. Simon, Adiabatic theorems for dense point spectra, Comm. Math. Phys. 128 (1990) 497-507.

[27] J. Avron, R. Seiler and B. Simon, Homotopy and quantization in condensed matter physics, Phys. Rev. Lett. 51 (1983) 51-53.

[28] J. Avron, R. Seiler and B. Simon, The index of a pair of projections, J. Funct. Anal. 120 (1994) 220-237.

[29] J. Avron, R. Seiler and L. G. Yaffe, Adiabatic theorems and applications to the quantum Hall effect, Commun. Math. Phys. 110 (1987) 33-49.

[30] D. Babbitt and E. Balslev, Local distortion techniques and unitarity of the $S$-matrix for the 2-body problem, J. Math. Anal. Appl. 54 (1976) 316-347.

[31] S. Bachmann, W. de Roeck and M. Fraas, The adiabatic theorem for many-body quantum systems, arXiv:math.SP:1612.01505.

[32] G. Baker, The theory and application of the Padé approximant method, Adv. Theoret. Phys. 1 (1965) 1-58.

[33] G. Baker, Essentials of Padé Approximants (Academic Press, New York, 1975).

[34] G. Baker and J. Gamel (eds), The Padé Approximant in Theoretical Physics (Academic Press, New York, 1970).

[35] A. A. Balinsky and W. D. Evans, Spectral Analysis of Relativistic Operators (Imperial College Press, London, 2011).

[36] E. Balslev, Analytic scattering theory of two-body Schrödinger operators, J. Funct. Anal. 29 (1978) 375-396.

[37] E. Balslev, Analytic scattering theory for many-body systems below the smallest three-body threshold, Comm. Math. Phys. 77 (1980) 173-210.

[38] E. Balslev, Analytic scattering theory of quantum mechanical three-body systems, Ann. Inst. H. Poincaré Sect. A 32 (1980) 125-160.

[39] E. Balslev and J. M. Combes, Spectral properties of many-body Schrödinger operators with dilation analytic interactions, Comm. Math. Phys. 22 (1971) 280-294.

[40] T. Banks, C. Bender and T. T. Wu, Coupled anharmonic oscillators, I. Equal-mass case, Phys. Rev. D 8 (1973) 3346-3366.

[41] H. Baumgärtel, Analytic Perturbation Theory for Matrices and Operators (Birkhauser, Boston, 1985).

[42] W. Beckner, Pitt's inequality and the uncertainty principle, Proc. Amer. Math. Soc. 123 (1995) 1897-1905.

[43] A. L. Belopol'skii and M. S. Birman, Existence of wave operators in scattering theory for a pair of spaces, Izv. Akad. Nauk SSSR Ser. Mat. 32 (1968) 1162-1175.

[44] M. Ben-Artzi and S. Klainerman, Decay and regularity for the Schrödinger equation, J. Anal. Math. 58 (1992) 25-37.

[45] L. Benassi and V. Grecchi, Resonances in the Stark effect and strongly asymptotic approximations, J. Phys. B 13 (1980) 911-924.

[46] C. Bender and T. T. Wu, Anharmonic oscillator, Phys. Rev. 184 (1969) 12311260. 
[47] C. Bender and T. T. Wu, Anharmonic oscillator, II. A study of perturbation theory in large order, Phys. Rev. D 7 (1973) 1620-1636.

[48] N. Benedikter, M. Porta and B. Schlein, Effective Evolution Equations from Quantum Dynamics, Springer Briefs in Mathematical Physics, Vol. 7 (Springer, Berlin, 2016).

[49] R. Benguria and E. H. Lieb, Proof of the stability of highly negative ions in the absence of the Pauli principle, Phys. Rev. Lett. 50 (1983) 1771-1774.

[50] M. V. Berry, Quantal phase factors accompanying adiabatic changes, Proc. Roy. Soc. A 392 (1984) 45-57.

[51] A. Beurling and J. Deny, Espaces de Dirichlet. I. Le cas élémentaire, Acta Math. 99 (1958) 203-224.

[52] M. S. Birman, Perturbation of the spectrum of a singular elliptic operator under variation of the boundary and boundary conditions, Dokl. Akad. Nauk SSSR 137 (1961) 761-763 [Soviet Math. Dokl. 2 (1961) 326-328].

[53] M. S. Birman, Perturbations of the continuous spectrum of a singular elliptic operator by varying the boundary and the boundary conditions, Vestnik Leningrad. Univ. 17 (1962) 22-55 [Amer. Math. Soc. Transl. Ser. 2, Vol. 225, eds. T. Suslina and D. Yafaev (American Mathematical Society, Providence, RI, 2008), pp. 19-53].

[54] M. Š. Birman, Conditions for the existence of wave operators, Dokl. Akad. Nauk SSSR 143 (1962) 506-509.

[55] M. Š. Birman, A criterion for existence of wave operators, Izv. Akad. Nauk. SSSR Ser. Mat. 27 (1963) 883-906 [Amer. Math. Soc. Transl. 54 (1966) 91-117].

[56] M. S. Birman, A local criterion for the existence of wave operators, Izv. Akad. Nauk SSSR Ser. Mat. 32 (1968) 914-942 [Math. USSR-Izv. 2 (1968) 879-906].

[57] M. ̌́. Birman, A test of the existence of complete wave operators in scattering theory for a pair of spaces, Probl. Math. Phys. 4 (1970) 22-26.

[58] M. Š. Birman and M. G. Krein, On the theory of wave operators and scattering operators, Dokl. Akad. Nauk. SSSR 144 (1962) 475-478 [Soviet Math. Dokl. 3 (1962) 740-744].

[59] D. Bollé, F. Gesztesy and C. Danneels, Threshold scattering in two dimensions, Ann. Inst. H. Poincaré Phys. Théor. 48 (1988) 175-204.

[60] D. Bollé, F. Gesztesy and M. Klaus, Scattering theory for one-dimensional systems with $\int d x V(x)=0$, J. Math. Anal. Appl. 122 (1987) 496-518, Errata 130 (1988) 590 .

[61] D. Bollé, F. Gesztesy and S. F. J. Wilk, A complete treatment of low-energy scattering in one dimension, J. Operator Theory 13 (1985) 3-31.

[62] E. Borel, Mémoire sur les séries divergentes, Ann. Sc. École Norm. Sup. 16 (1899) 9-131.

[63] G. Borg, On the point spectra of $y^{\prime \prime}+(A-q(x)) y=0$, Amer. J. Math. 73 (1951) 122-126.

[64] M. Born, Quantenmechanik der Stossvorgänge, Z. Phys. 38 (1926) 803-827.

[65] M. Born and V. A. Fock, Beweis des Adiabatensatzes, Z. Phys. A 51 (1928) 165180.

[66] A. Böttcher and I. Spitkovsky, A gentle guide to the basics of two projections theory, Linear Algebra Appl. 432 (2010) 1412-1459.

[67] A. Böttcher, I. Spitkovsky and B. Simon, Similarity between two projections, arXiv:1705.08937.

[68] H. J. Brascamp, E. H. Lieb and J. M. Luttinger, A general rearrangement inequality for multiple integrals, J. Funct. Anal. 17 (1974) 227-237. 
[69] O. Bratteli and D. W. Robinson, Operator Algebras and Quantum-Statistical Mechanics. II. Equilibrium States. Models in Quantum-Statistical Mechanics (Springer-Verlag, New York-Berlin, 1981).

[70] H. Brézis and T. Kato, Remarks on the Schrödinger operator with singular complex potentials, J. Math. Pures Appl. 58 (1979) 137-151.

[71] F. Brownell, Spectrum of the static potential Schrödinger equation over $E_{n}, A n n$. Math. 54 (1951) 554-594.

[72] F. Brownell, A note on Kato's uniqueness criterion for Schrödinger operator selfadjoint extensions, Pacific J. Math. 9 (1959) 953-973.

[73] L. Bruneau, J. Dereziński and V. Georgescu, Homogeneous Schrödinger operators on half-line, Ann. H. Poincaré 12 (2011) 547-590.

[74] J. Butler, Perturbation series for eigenvalues of analytic non-symmetric operators, Arch. Math. 10 (1959) 21-27.

[75] E. Caliceti, V. Grecchi and M. Maioli, The distributional Borel summability and the large coupling $\Phi^{4}$ lattice fields, Comm. Math. Phys. 104 (1986) 163-174.

[76] E. Caliceti, V. Grecchi and M. Maioli, Stark resonances: Asymptotics and distributional Borel sum, Comm. Math. Phys. 157 (1993) 347-357.

[77] C. Cancelier, A. Martinez and T. Ramond, Quantum resonances without analyticity, Asymptot. Anal. 44 (2005) 47-74.

[78] J. Cape, M. Tang and C. Priebe, The Kato-Temple inequality and eigenvalue concentration, arXiv:1603.06100.

[79] T. Carleman, Les Fonctions Quasianalytiques (Gauthier-Villars, Paris, 1926).

[80] T. Carleman, Sur un problème d'unicité pour les systèmes d'eq́uations aux derivées partielles à deux variables indépendantes, Ark. Mat. 26B (1939) 1-9.

[81] R. Carmona, Regularity properties of Schrödinger and Dirichlet semigroups, J. Funct. Anal. 17 (1974) 227-237.

[82] L. Cattaneo, G. M. Graf and W. Hunziker, A general resonance theory based on Mourre's inequality, Ann. Inst. H. Poincaré 7 (2006) 583-601.

[83] S. N. Chandler-Wilde and M. Lindner, Sufficiency of Favard's condition for a class of band-dominated operators on the axis, J. Funct. Anal. 254 (2008) 11461159.

[84] S. N. Chandler-Wilde and M. Lindner, Limit operators, collective compactness, and the spectral theory of infinite matrices, Mem. Amer. Math. Soc. 210 (2011) 989.

[85] P. Chernoff, Note on product formulas for operator semigroups, J. Funct. Anal. 2 (1968) 238-242.

[86] P. Chernoff, Semigroup product formulas and addition of unbounded operators, Bull. Amer. Math. Soc. 76 (1970) 395-398.

[87] P. Chernoff, Essential self-adjointness of powers of generators of hyperbolic equations, J. Funct. Anal. 12 (1973) 401-414.

[88] P. Chernoff, Product formulas, nonlinear semigroups, and addition of unbounded operators, Mem. Amer. Math. Soc. 140 (1974) 1-121.

[89] P. Chernoff, Schrödinger and Dirac operators with singular potentials and hyperbolic equations, Pacific J. Math. 72 (1977) 361-382.

[90] M. Christ and A. Kiselev, Absolutely continuous spectrum for one-dimensional Schrödinger operators with slowly decaying potentials: Some optimal results, J. Amer. Math. Soc. 11 (1998) 771-797.

[91] E. A. Coddington and N. Levinson, Theory of Ordinary Differential Equations (McGraw-Hill Book Company, Inc., New York-Toronto-London, 1955) [Reprint, Krieger Publishing Company, Malabar, FL, 1985]. 
[92] C. Conley and P. Rejto, Spectral concentration II, general theory, in Perturbation Theory and its Applications in Quantum Mechanics, ed., C. H. Wilcox (John Wiley \& Sons, New York, 1966), pp. 129-143.

[93] J. Cook, Convergence of the Møller wave matrix, J. Math. Phys. 36 (1957) 82-87.

[94] H. O. Cordes, With Tosio Kato at Berkeley, in Tosio Kato's Method and Principle for Evolution Equations in Mathematical Physics. Papers from the International Workshop held at Hokkaido University, Sapporo, June 27-29, 2001, eds. H. Fujita, S. T. Kuroda and H. Okamoto, pp. 1-17.

[95] H. O. Cordes, A. Jensen, S. T. Kuroda, G. Ponce, B. Simon and M. Taylor, Tosio Kato (1917-1999), Notices Amer. Math. Soc. 47 (2000) 650-657.

[96] H. L. Cycon, On the stability of selfadjointness of Schrödinger operators under positive perturbations, Proc. Roy. Soc. Edinburgh Sect. A 86 (1980) 165-173.

[97] H. L. Cycon, Resonances defined by modified dilations, Helv. Phys. Acta 58 (1985) 969-981.

[98] H. Cycon, R. Froese, W. Kirsch and B. Simon, Schrödinger Operators with Application to Quantum Mechanics and Global Geometry (Springer-Verlag, Berlin, 1987).

[99] E. B. Davies, A model for absorption or decay, Helv. Phys. Acta 48 (1975) 365-382.

[100] E. B. Davies, One-Parameter Semigroups (Academic Press, London, 1980).

[101] E. B. Davies and A. M. Hinz, Kato class potentials for higher order elliptic operators, J. London Math. Soc. 58 (1998) 669-678.

[102] C. Davis, Estimating eigenvalues, Proc. Amer. Math. Soc. 3 (1952) 942-947.

[103] C. Davis, Separation of two linear subspaces, Acta Sci. Math. (Szeged) 16 (1958) $172-187$.

[104] L. deBranges, Perturbation of self-adjoint transformations, Amer. J. Mach. 84 (1962) 543-580.

[105] P. Deift and R. Killip, On the absolutely continuous spectrum of one-dimensional Schrödinger operators with square summable potentials, Comm. Math. Phys. 203 (1999) 341-347.

[106] P. Deift and B. Simon, On the decoupling of finite singularities from the question of asymptotic completeness in two body quantum systems, J. Funct. Anal. 23 (1976) 218-238.

[107] P. Deift and B. Simon, A time-dependent approach to the completeness of multiparticle quantum systems, Comm. Pure Appl. Math. 30 (1977) 573-583.

[108] D. Del Pasqua, Su una nozione di varietà lineari disgiunte di uno spazio di Banach (On a notion of disjoint linear manifolds of a Banach space), Rend. Mat. Appl. 5 (1955) 406-422.

[109] R. del Rio, S. Jitomirskaya, Y. Last and B. Simon, Operators with singular continuous spectrum, IV. Hausdorff dimensions, rank one perturbations, and localization, J. Anal. Math. 69 (1996) 153-200.

[110] R. del Rio, N. Makarov and B. Simon, Operators with singular continuous spectrum: II. Rank one operators, Comm. Math. Phys. 165 (1994) 59-67.

[111] Q. Deng, Y. Ding and X. Yao, Maximal and minimal forms for generalized Schrödinger operators, Indiana Univ. Math. J. 63 (2014) 727-738.

[112] J. Dereziński, Asymptotic completeness of long-range $N$-body quantum systems, Ann. Math. 138 (1993) 427-476.

[113] J. Dereziński and C. Gérard, Scattering Theory of Classical and Quantum N-Particle Systems (Springer, New York, 1997), http://www.fuw.edu.pl/ derezins/bookn.pdf.

[114] A. Devinatz, Essential self-adjointness of Schrödinger-type operators, J. Funct. Anal. 25 (1977) 58-69. 
[115] V. Dinu, A. Jensen and G. Nenciu, Nonexponential decay laws in perturbation theory of near threshold eigenvalues, J. Math. Phys. 50 (2009) 013516.

[116] V. Dinu, A. Jensen and G. Nenciu, Perturbation of near threshold eigenvalues: Crossover from exponential to non-exponential decay laws, Rev. Math. Phys. 23 (2011) 83-125.

[117] J. Dixmier, Position relative de deux variétés linéaires fermées dans un espace de Hilbert, Revue Sci. 86 (1948) 387-399.

[118] J. Dollard, Asymptotic convergence and the Coulomb interaction, J. Math. Phys. 5 (1964) 729-738.

[119] C. Dolph and J. Howland, Dedication of special issue in honor of Otto Laporte and Tosio Kato, J. Math. Anal. Appl. 127 (1987) 299-311.

[120] W. F. Donoghue, On the perturbation of spectra, Comm. Pure Appl. Math. 18 (1965) 559-579.

[121] Y. N. Dou, W. J. Shi, M. M. Cui and H. K. Du, General explicit expressions for intertwining operators and direct rotations of two orthogonal projections, arXiv:1705.05870v1.

[122] N. Dunford, Spectral theory, Bull. Amer. Math. Soc. 49 (1943) 637-651.

[123] N. Dunford, Spectral theory. I. Convergence to projections, Trans. Amer. Math. Soc. 54 (1943) 185-217.

[124] S. Dyatlov and M. Zworski, Mathematical Theory of Scattering Resonances, book in preparation.

[125] F. Dyson, Divergence of perturbation theory in quantum electrodynamics, Phys. Rev. 85 (1952) 631-632.

[126] M. S. P. Eastham, On the absence of square-integrable solutions of the SturmLiouville equation, in Ordinary and Partial Differential Equations, Dundee 1976, Lecture Notes in Mathematics, eds. W. M. Everitt and B. D. Sleeman, Vol. 564 (Springer, Berlin, 1976).

[127] M. S. P. Eastham, W. D. Evans and J. B. McLeod, The essential self-adjointness of Schrödinger-type operators, Arch. Ration. Mech. Anal. 60 (1976) 185-204.

[128] M. S. P. Eastham and H. Kalf, Schrödinger-type Operators with Continuous Spectra, Research Notes in Mathematics (Pitman, Boston-London, 1982).

[129] W. D. Evans, On the essential self-adjointness of powers of Schrödinger-type operators, Proc. Roy. Soc. Edinburgh 79A (1977) 61-77.

[130] J. P. Eckmann, J. Magnen and R, Sénéor, Decay properties and Borel summability for the Schwinger functions in $P(\varphi)_{2}$ theories, Comm. Math. Phys. 39 (1975) 251271.

[131] E. G. Effros, Why the circle is connected: An introduction to quantized topology, Math. Intelligencer 11(1) (1989) 27-34.

[132] V. Efimov, Energy levels arising from resonant two-body forces in a three-body system, Phys. Lett. B 33 (1970) 563-564.

[133] P. Ehrenfest, Adiabatische invarianten und quantentheorie, Ann. Phys. 51 (1916) $327-352$.

[134] A. Elgart and G. A. Hagedorn, A note on the switching adiabatic theorem, J. Math. Phys. 53 (2012) 102202.

[135] V. Enss, A note on Hunziker's theorem, Comm. Math. Phys. 52 (1977) 233-238.

[136] V. Enss, Asymptotic completeness for quantum-mechanical potential scattering, I. Short-range potentials, Comm. Math. Phys. 61 (1978) 285-291.

[137] V. Enss, Completeness of Three-Body Quantum Scattering, Lecture Notes in Mathematics, eds. Ph. Blanchard and L. Streit, Vol. 1031 (Springer-Verlag, Berlin, 1983), pp. $62-88$. 
[138] P. Epstein, Zur Theorie des Starkeffektes, Ann. Phys. 50 (1916) 489-520.

[139] P. Epstein, The Stark effect from the point of view of Schroedinger's quantum theory, Phys. Rev. 28 (1926) 695-710.

[140] M. J. Esteban, M. Lewin and E. Séré, Variational methods in relativistic quantum mechanics, Bull. Amer. Math. Soc. 45 (2008) 535-593.

[141] M. J. Esteban and M. Loss, Self-adjointness for Dirac operators via Hardy-Dirac inequalities, J. Math. Phys. 48 (2007) 112107.

[142] C. Estienne, M. Busuttil, A. Moini and G. Drake, Critical nuclear charge for twoelectron atoms, Phys. Rev. Lett. 112 (2014) 1-5.

[143] W. Faris, The product formula for semigroups defined by Friedrichs extensions, Pacific J. Math. 22 (1967) 47-70.

[144] W. G. Faris, Self-Adjoint Operators, Lecture Notes in Mathematics, Vol. 433 (Springer-Verlag, Berlin-New York, 1975).

[145] W. Faris and R. Lavine, Commutators and self-adjointness of Hamiltonian operators, Comm. Math. Phys. 35 (1974) 39-48.

[146] H. Feshbach, Unified theory of nuclear reactions, I, II, Ann. Phys. 5 (1958) 357-390; 19 (1962) 287-313.

[147] T. Figiel and W. B. Johnson, The Lidskii trace property and the nest approximation property in Banach spaces, J. Funct. Anal. 271 (2016) 566-576.

[148] V. A. Fock, On the Schrödinger equation of the helium atom. I, II, Norske Vid. Selsk. Forh. Trondheim 31 (1958) 138-151 [Russian original: Izv. Akad. Nauk SSSR Ser. Fiz. 18 (1954) 161-172].

[149] S. Fournais, M. Hoffmann-Ostenhof, T. Hoffmann-Ostenhof and T. Østergaard Sørensen, The electron density is smooth away from the nuclei, Comm. Math. Phys. 228 (2002) 401-415.

[150] S. Fournais, M. Hoffmann-Ostenhof, T. Hoffmann-Ostenhof and T. Østergaard Sørensen, Analyticity of the density of electronic wave functions, Ark. Math. 42 (2004) 87-106.

[151] S. Fournais, M. Hoffmann-Ostenhof, T. Hoffmann-Ostenhof and T. Østergaard Sørensen, Sharp regularity for Coulombic many-electron wave functions, Comm. Math. Phys. 255 (2005) 183-227.

[152] S. Fournais, M. Hoffmann-Ostenhof, T. Hoffmann-Ostenhof and T. Østergaard Sørensen, Analytic structure of many-body Coulombic wave functions, Comm. Math. Phys. 289 (2009) 291-310.

[153] S. Fournais and E. Skibsted, Zero energy asymptotics of the resolvent for a class of slowly decaying potentials, Math. Z. 248 (2004) 593-633.

[154] R. L. Frank, J. Bellazzini, E. H. Lieb and R. Seiringer, Existence of ground states for negative ions at the binding threshold, Rev. Math. Phys. 26 (2014) 1350021.

[155] R. L. Frank, A. Laptev and T. Weidl, Lieb-Thirring Inequalities, book in preparation.

[156] R. L. Frank and E. H. Lieb, A new, rearrangement-free proof of the sharp HardyLittlewood-Sobolev inequality, in Spectral Theory, Function Spaces and Inequalities, eds. B. M. Brown, J. Lang and I. G. Wood (Birkhäuser/Springer, Basel, 2012), pp. $55-67$.

[157] R. L. Frank, E. H. Lieb and R. Seiringer, Hardy-Lieb-Thirring inequalities for fractional Schrödinger operators, J. Amer. Math. Soc. 21 (2008) 925-950.

[158] R. L. Frank, E. H. Lieb and R. Seiringer, Binding of polarons and atoms at threshold, Comm. Math. Phys. 313 (2012) 405-424.

[159] R. L. Frank and R. Seiringer, Non-linear ground state representations and sharp Hardy inequalities, J. Funct. Anal. 255 (2008) 3407-3430. 
[160] R. L. Frank and B. Simon, Eigenvalue bounds for Schrödinger operators with complex potentials. II, J. Spectr. Theory 7 (2017) 633-658.

[161] J. Frehse, Essential selfadjointness of singular elliptic operators, Bol. Soc. Brasil. Mat. 8 (1977) 87-107.

[162] H. Freudenthal, Über die Friedrichssche Fortsetzung halbbeschränkter Hermitescher Operatoren, Proc. Akad. Wet. Amsterdam 39 (1936) 832-833.

[163] K. O. Friedrichs, Spektraltheorie halbbeschränkter Operatoren und Anwendung auf die Spektralzerlegung von Differentialoperatoren. I, II, Math. Ann. 109 (1934) 46587, 685-713; 110 (1934/35) 777-779.

[164] K. O. Friedrichs, Über die spektralzerlegung eines integraloperators, Math. Ann. 115 (1938) 249-272.

[165] K. O. Friedrichs, On differential operators in Hilbert spaces, Amer. J. Math. 61 (1939) 523-544.

[166] K. O. Friedrichs, On the perturbation of continuous spectra, Comm. Pure Appl. Math. 1 (1948) 361-406.

[167] K. O. Friedrichs, Symmetric hyperbolic linear differential equations, Comm. Pure Appl. Math. 7 (1954) 345-392.

[168] K. O. Friedrichs, Perturbation of Spectra in Hilbert Space (American Mathematical Society, Providence, RI, 1965).

[169] K. O. Friedrichs and P. Rejto, On a perturbation through which a discrete spectrum becomes continuous, Comm. Pure Appl. Math. 15 (1962) 219-235.

[170] R. Froese and I. Herbst, Exponential bounds and absence of positive eigenvalues for N-body Schrödinger operators, Comm. Math. Phys. 87 (1982) 429-447.

[171] R. Froese and I. Herbst, A new proof of the Mourre estimate, Duke Math. J. 49 (1982) 1075-1085.

[172] R. Froese, I. Herbst, M. Hoffmann-Ostenhof and T. Hoffmann-Ostenhof, On the absence of positive eigenvalues for one-body Schrödinger operators, J. Anal. Math. 41 (1982) 272-284.

[173] R. Froese, I. Herbst, M. Hoffmann-Ostenhof and T. Hoffmann-Ostenhof, $L^{2}$-exponential lower bounds to solutions of the Schrödinger equation, Commun. Math. Phys. 87 (1982) 265-286.

[174] J. Fujii, M. Fujii, T. Furuta and R. Nakamoto, Norm inequalities equivalent to Heinz inequality, Proc. Amer. Math. Soc. 118 (1993) 827-830.

[175] H. Fujita, H. Okamoto and S. T. Kuroda, Edited correspondence of T. Kato and with E. C. Kemble and J. von Neumann, in preparation.

[176] L. Gårding, On the essential spectrum of Schrödinger operators, J. Funct. Anal. 52 (1983) 1-10.

[177] Y. Gâtel and D. Yafaev, On solutions of the Schrödinger equation with radiation conditions at infinity: The long-range case, Ann. Inst. Fourier (Grenoble) 49 (1999) $1581-1602$.

[178] I. M. Gel'fand, Normierte ringe, Rec. Math. [Mat. Sb.] N.S. 9 (1941) 3-24.

[179] M. Gell'Mann and M. L. Goldberger, The formal theory of scattering, Phys. Rev. 91 (1953) 398-408.

[180] V. Georgescu and A. Iftimovici, Crossed products of $C^{*}$-algebras and spectral analysis of quantum Hamiltonians, Comm. Math. Phys. 228 (2002) 519-560.

[181] C. Gérard, Distortion analyticity for $N$-particle Hamiltonians, Helv. Phys. Acta 66 (1993) 216-225.

[182] F. Gesztesy and L. L. Littlejohn, Factorizations and Hardy-Rellich-type inequalities, in Partial Differential Equations, Mathematical Physics, and Stochastic Analysis. A Volume in Honor of Helge Holden's 60th Birthday, EMS Congress Reports, eds. F. 
Gesztesy, H. Hanche-Olsen, E. Jakobsen, Y. Lyubarskii, N. Risebro and K. Seip, to appear.

[183] F. Gesztesy, M. Mitrea, I. Nenciu and G. Teschl, Decoupling of deficiency indices and applications to Schrödinger-type operators with possibly strongly singular potentials, Adv. Math. 301 (2016) 1022-1061.

[184] D. Gilbarg and N. S. Trudinger, Elliptic Partial Differential Equations of Second Order (Springer, Berlin, Heidelberg, New York, 2001).

[185] J. Glimm, Boson fields with nonlinear self-interaction in two dimensions, Comm. Math. Phys. 8 (1968) 12-25.

[186] J. Glimm and A. Jaffe, A $\lambda\left(\varphi^{4}\right)_{2}$ quantum field theory without cutoffs, I, Phys. Rev. 176 (1968) 1945-1951.

[187] J. Glimm and A. Jaffe, The $\lambda\left(\varphi^{4}\right)_{2}$ quantum field theory without cutoffs, IV: Perturbation of the Hamiltonian, J. Math. Phys. 11 (1972) 1568-1584.

[188] J. Glimm and A. Jaffe, Quantum Physics: A Functional Integral Point of View, 2nd edn. (Springer-Verlag, New York, 1987).

[189] G. Golub and H. van der Vorst, Eigenvalue computation in the 20th century, J. Comput. Appl. Math. 123 (2000) 35-65.

[190] G. M. Graf, Asymptotic completeness for $N$-body short-range quantum systems: A new proof, Comm. Math. Phys. 132 (1990) 73-101.

[191] G. M. Graf and D. Schenker, Classical action and quantum $N$-body asymptotic completeness, in Multiparticle Quantum Scattering with Applications to Nuclear, Atomic and Molecular Physics, eds. D. G. Truhlar and B. Simon (Springer, New York, 1997), pp. 103-119.

[192] S. Graffi and V. Grecchi, Resonances in Stark effect and perturbation theory, Comm. Math. Phys. 62 (1978) 83-96.

[193] S. Graffi and V. Grecchi, Existence and Borel summability of Resonances in hydrogen stark effect, Lett. Math. Phys. 3 (1978) 336-340.

[194] S. Graffi and V. Grecchi, On a relation between Stieltjes and Borel summabilities, J. Math. Phys. 19 (1978) 1002-1007.

[195] S. Graffi and V. Grecchi, Confinement of the resonances in hydrogen Stark effect, J. Phys. B 12 (1979) L265-L267.

[196] S. Graffi and V. Grecchi, Resonances in the Stark effect of atomic systems, Comm. Math. Phys. 79 (1981) 91-109.

[197] S. Graffi, V. Grecchi, S. Levoni and M. Maioli, Resonances in one-dimensional Stark effect and continued fractions, J. Math. Phys. 20 (1979) 685-690.

[198] S. Graffi, V. Grecchi and B. Simon, Borel summability: Application to the anharmonic oscillator, Phys. Lett. D 32 (1970) 631-634.

[199] S. Graffi, V. Grecchi and B. Simon, Complete separability of the Stark effect in hydrogen, J. Phys. A 12 (1979) L193-L195.

[200] D. Gridnev, Bound states at threshold resulting from Coulomb repulsion, J. Math. Phys. 53 (2012) 102108.

[201] D. Griffiths, Introduction to Quantum Mechanics (Pearson Prentice Hall, Upper Saddle River, 2004).

[202] A. Grossman and T. T. Wu, Schrödinger scattering amplitude, I, III, J. Math. Phys. 2 (1961) 710-713; 3 (1962) 684-689.

[203] B. Güneysu and O. Post, Path integrals and the essential self-adjointness of differential operators on noncompact manifolds, Math. Z. 275 (2013) 331348.

[204] V. I. Gurarii, Openings and inclinations of subspaces of a Banach space, Teor. Funkc. Funkc. Anal. ih Priloz. 1 (1965) 194-204. 
[205] S. Gustafson and I. M Sigal, Mathematical Concepts of Quantum Mechanics, 2nd edn. (Springer, Heidelberg, 2011).

[206] M. Hack, On the convergence to the Møller wave operators, Nuovo Cimento 9 (1958) $731-733$.

[207] G. A. Hagedorn, A link between scattering resonances and dilation analytic resonances in few body quantum mechanics, Comm. Math. Phys. 65 (1979) 181-188.

[208] G. A. Hagedorn, Proof of the Landau-Zener formula in an adiabatic limit with small eigenvalue gaps, Comm. Math. Phys. 136 (1991) 433-449.

[209] P. Halmos, Two subspaces, Trans. Amer. Math. Soc. 144 (1969) 381-389.

[210] P. R. Halmos and S. Kakutani, Products of symmetries, Bull. Amer. Math. Soc. 64 (1958) $77-78$.

[211] H. Halpern, MathSciNet review of [28]; MR1262254 (1995).

[212] G. H. Hardy, Divergent Series (Oxford University Press, London and New York, 1949).

[213] G. H. Hardy, J. E. Littlewood and G. Pólya, Inequalities, Cambridge Mathematical Library (Cambridge University Press, Cambridge, 1988).

[214] E. Harrell, Generalizations of Temple's inequality, Proc. Amer. Math. Soc. 69 (1978) $271-276$.

[215] E. Harrell and B. Simon, The mathematical theory of resonances whose widths are exponentially small, Duke Math. J. 47 (1980) 845-902.

[216] P. Hartman and A. Wintner, A criterion for the non-degeneracy of the wave equation, Amer. J. Math. 71 (1949) 206-213.

[217] M. B. Hastings and X.-G. Wen, Quasiadiabatic continuation of quantum states: The stability of topological ground-state degeneracy and emergent gauge invariance, Phys. Rev. B $\mathbf{7 2}$ (2005) 045141.

[218] E. Heinz, Beiträge zur Störungstheorie der Spektralzerlegung, Math. Ann. 123 (1951) 415-438.

[219] W. Heisenberg, Die "beobachtbaren Grössen" in der theorie der elementarteilchen, I, II, Z. Phys. 120 (1943) 513-538, 673-702.

[220] B. Helffer and J. Sjöstrand, Multiple wells in the semiclassical limit. I-VI, Comm. Partial Differential Equations 9 (1984) 337-408; Ann. Inst. H. Poincaré Phys. Théor. 42 (1985) 127-212; Math. Nachr. 124 (1985) 263-313; Comm. Partial Differential Equations 10 (1985) 245-340; Current Topics in Partial Differential Equations (Kinokuniya, Tokyo, 1986), pp. 133-186; Ann. Inst. H. Poincaré Phys. Théor. 46 (1987) 353-372.

[221] B. Helffer and J. Sjöstrand, Résonances en limite semi-classique [Resonances in the semiclassical limit], Mém. Soc. Math. France (N.S.) 24-25 (1986) 1-228.

[222] B. Hellwig, Ein kriterium für die Selbstadjungiertheit elliptischer differentialoperatoren im $R_{n}$, Math. Z. 86 (1964) 255-262.

[223] B. Hellwig, Ein kriterium für die Selbstadjungiertheit singulärer elliptischer differentialoperatoren im Gebiet G, Math. Z. 89 (1965) 333-344.

[224] B. Hellwig, A criterion for self-adjointness of singular elliptic differential operators, J. Math. Anal. Appl. 26 (1969) 279-291.

[225] J. Henrard, The adiabatic invariant in classical mechanics, in Dynamics Reported: Expositions in Dynamical Systems, Vol. 2 (Springer, Berlin, 1993), pp. 117-235.

[226] I. Herbst, Spectral theory of the operator $\left(p^{2}+m^{2}\right)^{1 / 2}-Z e^{2} / r$, Comm. Math. Phys. 53 (1977) 285-294.

[227] I. Herbst, Unitary equivalence of Stark Hamiltonians, Math. Z. 155 (1977) 55-71.

[228] I. Herbst, Dilation analyticity in constant electric field, I: The two body problem, Comm. Math. Phys. 64 (1979) 279-298. 
[229] I. Herbst, J. S. Møller and E. Skibsted, Spectral analysis of N-body stark hamiltonians, Comm. Math. Phys. 174 (1995) 261-294.

[230] I. Herbst and B. Simon, Some remarkable examples in eigenvalue perturbation theory, Phys. Lett. B 78 (1978) 304-306.

[231] I. Herbst and B. Simon, Dilation analyticity in constant electric field, II: The $N$-body problem, Borel summability, Comm. Math. Phys. 80 (1981) 181-216.

[232] I. Herbst and A. Sloan, Perturbations of translation invariant positivity preserving semigroups in $L^{2}(\mathbb{R})$, Trans. Amer. Math. Soc. 236 (1978) 325-360.

[233] C. Hermite, Sur la function exponentielle, C. R. Acad. Sci. 76 (1873) 18-24, 74-79, 226-233, 285-293.

[234] H. Hess, R. Schrader and D. A. Uhlenbrock, Domination of semigroups and generalization of Kato's inequality, Duke Math. J. 44 (1977) 893-904.

[235] F. N. Hill, On the analytic structure of the wavefunction of a hydrogen atom in an analytic potential, J. Math. Phys. 25 (1984) 1577-1583.

[236] D. Hirsbrunner and J. Loeffel, Sur les séries asymptotiques sommables selon Borel, Helv. Phys. Acta 48 (1975) 546.

[237] M. Hoffmann-Ostenhof, T. Hoffmann-Ostenhof and T. Østergaard Sørensen, Electron wavefunctions and densities for atoms, Ann. Inst. H. Poincaré 2 (2011) 77-100.

[238] M. Hoffmann-Ostenhof, T. Hoffmann-Ostenhof and B. Simon, A multiparticle Coulomb system with bound state at threshold, J. Phys. A 16 (1983) 11251131.

[239] L. Hörmander, The Analysis of Linear Partial Differential Operators, II. Differential Operators with Constant Coefficients (Springer, Berlin, 2005).

[240] L. Hörmander, The Analysis of Linear Partial Differential Operators, IV. Fourier Integral Operators (Springer, Berlin, 2009).

[241] J. Howland, Banach space techniques in the perturbation theory of self-adjoint operators with continuous spectra, J. Math. Anal. Appl. 20 (1967) 22-47.

[242] J. Howland, Spectral concentration and virtual poles, Amer. J. Math. 91 (1969) 1106-1126.

[243] J. Howland, Embedded eigenvalues and virtual poles, Pacific J. Math. 29 (1969) $565-582$.

[244] J. Howland, Spectral concentration and virtual poles, II, Trans. Amer. Math. Soc. 162 (1971) 141-156.

[245] J. Howland, Perturbation of embedded eigenvalues, Bull. Amer. Math. Soc. 78 (1972) 280-283.

[246] J. Howland, Puiseux series for resonances at an embedded eigenvalue, Pacific J. Math. 55 (1974) 157-176.

[247] D. Huet, Phénomènes de perturbation singulière dans les problèmes aux limites, Ann. Inst. Fourier. Grenoble 10 (1960) 61-150.

[248] D. S. Hughes and C. Eckart, The effect of the motion of the nucleus on the spectra of Li I and Li II, Phys. Rev. 36 (1930) 694-698.

[249] W. Hunziker, On the spectra of Schrödinger multiparticle Hamiltonians, Helv. Phys. Acta 39 (1966) 451-462.

[250] W. Hunziker, Distortion analyticity and molecular resonance curves, Ann. Inst. H. Poincaré Phys. Théor. 45 (1986) 339-358.

[251] W. Hunziker and I. M. Sigal, The quantum N-body problem, J. Math. Phys. 41 (2000) 3448-3510.

[252] T. Ikebe, Eigenfunction expansions associated with the Schroedinger operators and their applications to scattering theory, Arch. Rational Mech. Anal. 5 (1960) 1-34. 
[253] T. Ikebe, Remarks on the orthogonality of eigenfunctions for the Schrödinger operator in $R^{n}$, J. Fac. Sci. Univ. Tokyo Sect. I 17 (1970) 355-361.

[254] T. Ikebe and T. Kato, Application of variational method to the Thomas-Fermi equation, J. Phys. Soc. Japan 12 (1957) 201-203.

[255] T. Ikebe and T. Kato, Uniqueness of the self-adjoint extensions of singular elliptic differential operators, Arch. Rational Mech. Anal. 9 (1962) 77-92.

[256] T. Ikebe and J. Uchiyama, On the asymptotic behavior of eigenfunctions of secondorder elliptic operators, J. Math. Kyoto Univ. 11 (1971) 425-448.

[257] A. D. Ionescu and D. Jerison, On the absence of positive eigenvalues of Schrödinger operators with rough potentials, Geom. Funct. Anal. 13 (2003) 1029-1081.

[258] A. D. Ionescu and W. Schlag, Agmon-Kato-Kuroda theorems for a large class of perturbations, Duke Math. J. 131 (2006) 397-440.

[259] R. J. Iorio and M. O'Carroll, Asymptotic completeness for multi-particle Schroedinger Hamiltonians with weak potentials, Comm. Math. Phys. 27 (1972) $137-145$.

[260] R. Ismagilov, Conditions for the semiboundedness and discreteness of the spectrum for one-dimensional differential equations, Sov. Math. Dokl. 2 (1961) 11371140 .

[261] H. Isozaki and H. Kitada, Modified wave operators with time-independent modifiers, J. Fac. Sci. Univ. Tokyo Sect. IA Math. 32 (1985) 77-104.

[262] K. Ito and A. Jensen, A complete classification of threshold properties for onedimensional discrete Schrödinger operators, Rev. Math. Phys. 27 (2015) 1550002.

[263] C. Jacobi, Vorlesungen über Dynamik (G. Reiner, Berlin, 1884).

[264] W. Jäger, Zur Theorie der Schwingungsgleichung mit variablen Koeffizienten in Aussengebieten, Math. Z. 102 (1967) 62-88.

[265] V. Jakšić and J. Segert, Exponential approach to the adiabatic limit and the Landau-Zener formula, Rev. Math. Phys. 4 (1992) 529-574.

[266] V. Jakšić and J. Segert, On the Landau-Zener formula for two-level systems, J. Math. Phys. 34 (1993) 2807-2820.

[267] S. Jansen, M. B. Ruskai and R. Seiler, Bounds for the adiabatic approximation with applications to quantum computation, J. Math. Phys. 48 (2007) 102111-102126.

[268] R. Jastrow, Many-body problem with strong forces, Phys. Rev. 98 (1955) 14791484.

[269] J. M. Jauch, Theory of the scattering operator, I, II, Helv. Phys. Acta. 31 (1958) $127-158,661-684$.

[270] J. M. Jauch and I. I. Zinnes, The asymptotic condition for simple scattering systems, Nuovo Cimento 11 (1959) 553-567.

[271] T. Jecko, A new proof of the analyticity of the electron density, Lett. Math. Phys. 93 (2010) 73-83.

[272] A. Jensen, Local distortion technique, resonances, and poles of the $S$-matrix, J. Math. Anal. Appl. 59 (1977) 505-513.

[273] A. Jensen, Spectral properties of Schrödinger operators and time-decay of the wave functions results in $L^{2}\left(\mathbb{R}^{m}\right), m \geq 5$, Duke Math. J. 47 (1980) 57-80.

[274] A. Jensen, Spectral properties of Schrödinger operators and time-decay of the wave functions. Results in $L^{2}\left(\mathbb{R}^{4}\right)$, J. Math. Anal. Appl. 101 (1984) 397422 .

[275] A. Jensen and T. Kato, Spectral properties of Schrödinger operators and time-decay of the wave functions, Duke Math. J. 46 (1979) 583-611.

[276] A. Jensen and G. Nenciu, A unified approach to resolvent expansions at thresholds, Rev. Math. Phys. 13 (2001) 717-754. 
[277] A. Jensen and G. Nenciu, The Fermi golden rule and its form at thresholds in odd dimensions, Comm. Math. Phys. 261 (2006) 693-727.

[278] D. Jerison and C. E. Kenig, Unique continuation and absence of positive eigenvalues for Schrödinger operators, Ann. Math. 121 (1985) 463-494.

[279] S. Jitomirskaya and B. Simon, Operators with singular continuous spectrum: III. Almost periodic Schrödinger operators, Comm. Math. Phys. 165 (1994) 201-205.

[280] K. Jörgens, Perturbations of the Dirac operator, in Proc. Dundee Conf. Differential Equations, eds. W. N. Everitt and B. D. Sleeman (Springer, Berlin-Heidelberg-New York, 1972), pp. 87-102.

[281] J.-L. Journé, A. Soffer and C. D. Sogge, Decay estimates for Schrödinger operators, Comm. Pure Appl. Math. 44 (1991) 573-604.

[282] A. Joye, General adiabatic evolution with a gap condition, Comm. Math. Phys. 275 (2007) 139-162.

[283] A. Joye and C. E. Pfister, Exponentially small adiabatic invariant for the Schrödinger equation, Comm. Math. Phys. 140 (1991) 15-41.

[284] H. Kalf, Self-adjointness for strongly singular potentials with $a-|x|^{2}$ fall-off at infinity, Math. Z. 133 (1973) 249-255.

[285] H. Kalf, The quantum mechanical virial theorem and the absence of positive energy bound states of Schrödinger operators, Israel J. Math. 20 (1975) 57-69.

[286] H. Kalf, Gauss's theorem and the self-adjointness of Schrödinger operators, Ark. Mat. 18 (1980) 19-47.

[287] H. Kalf, U.-W. Schmincke, J. Walter and R. Wüst, On the spectral theory of Schrödinger and Dirac operators with strongly singular potentials, in Spectral Theory and Differential Equations, Lecture Notes in Mathematics, ed. W. N. Everitt Vol. 448 (Springer, Berlin, 1975), pp. 182-226.

[288] H. Kalf and V. Krishna Kumar, On the absence of positive eigenvalues of Schrödinger operators with long range potentials, Trans. Amer. Math. Soc. 275 (1983) 215-229.

[289] H. Kalf and J. Walter, Strongly singular potentials and essential self-adjointness of singular elliptic operators in $C_{0}^{\infty}\left(\mathbb{R}^{\nu} \backslash\{0\}\right)$, J. Funct. Anal. 10 (1972) 114130 .

[290] H. Kalf and J. Walter, Note on a paper of Simon on essentially self-adjoint Schrödinger operators with singular potentials, Arch. Rational Mech. Anal. 52 (1973) 258-260.

[291] N. J. Kalton, A note on pairs of projections, Bol. Soc. Mat. Mexicana 3 (1997) 309-311.

[292] B. Karnarski, Generalized dirac-operators with several singularities, J. Operator Theory 13 (1985) 171-188.

[293] T. Kato, Examples in which the perturbation method fails, Prog. Theor. Phys. 3 (1948) 313-314.

[294] T. Kato, On the upper and lower bounds of eigenvalues, J. Phys. Soc. Japan 4 (1949) 334-339.

[295] T. Kato, On the convergence of the perturbation method, I, Prog. Theor. Phys. 4 (1949) 514-523.

[296] T. Kato, On the convergence of the perturbation method, II, Prog. Theor. Phys. 5 (1950) 95-101, 207-212.

[297] T. Kato, Perturbation theory for linear operators (in Japanese), Sûgaku Math. 2 (1950) 201-208.

[298] T. Kato, Variational methods in collision problems, Phys. Rev. 80 (1950) 475.

[299] T. Kato, Upper and lower bounds of eigenvalues, Phys. Rev. 77 (1950) 413. 
[300] T. Kato, On the adiabatic theorem for quantum mechanics, J. Phys. Soc. Japan 5 (1950) 435-439.

[301] T. Kato, Fundamental properties of Hamiltonian operators of Schrödinger type, Trans. Amer. Math. Soc. 70 (1951) 195-211.

[302] T. Kato, On the existence of solutions of the helium wave equation, Trans. Amer. Math. Soc. 70 (1951) 212-218.

[303] T. Kato, On the convergence of the perturbation method, J. Fac. Sci. Univ. Tokyo 6 (1951) 145-226.

[304] T. Kato, Note on Schwinger's variational method, Prog. Theor. Phys. 6 (1951) 295305.

[305] T. Kato, Upper and lower bounds of scattering phases, Prog. Theore. Phys. 6 (1951) 394-407.

[306] T. Kato, Notes on some inequalities for linear operators, Math. Ann. 125 (1952) $208-212$.

[307] T. Kato, On the perturbation theory of closed linear operators, J. Math. Soc. Japan 4 (1952) 323-337.

[308] T. Kato, On some approximate methods concerning the operators $T^{*} T$, Math. Ann. 126 (1953) 253-262.

[309] T. Kato, Perturbation theory of semi-bounded operators, Math. Ann. 125 (1953) 435-447.

[310] T. Kato, Quadratic forms in Hilbert space and asymptotic perturbation series, Technical Report No. 7, University of California, Berkley, 1955.

[311] T. Kato, Notes on projections and perturbation theory, Technical Report No. 9, University of California, Berkley, 1955.

[312] T. Kato, On the eigenfunctions of many particle systems in quantum mechanics, Comm. Pure Appl. Math. 10 (1957) 151-177.

[313] T. Kato, On finite-dimensional perturbations of self-adjoint operators, J. Math. Soc. Japan 9 (1957) 239-249.

[314] T. Kato, Perturbation of continuous spectra by trace class operators, Proc. Japan Acad. 33 (1957) 260-264.

[315] T. Kato, Perturbation of a scattering operator and its continuous spectrum (in Japanese), Sugaku 9 (1957) 75-84.

[316] T. Kato, Non-existence of bound states with positive energy, J. Phys. Soc. Japan 14 (1959) 382.

[317] T. Kato, Growth properties of solutions of the reduced wave equation with a variable coefficient, Comm. Pure Appl. Math. 12 (1959) 403-425.

[318] T. Kato, Remarks on pseudo-resolvents and infinitesimal generators of semi-groups, Proc. Japan Acad. 35 (1959) 467-468.

[319] T. Kato, Estimation of iterated matrices, with application to the von Neumann condition, Numer. Math. 2 (1960) 22-29.

[320] T. Kato, A generalization of the Heinz inequality, Proc. Japan Acad. Ser. A. Math. Sci. 6 (1961) 305-308.

[321] T. Kato, Wave operators and unitary equivalence, Pacific J. Math. 15 (1965) 171180.

[322] T. Kato, Wave operators and similarity for some non-selfadjoint operators, Math. Ann. 162 (1966) 258-279.

[323] T. Kato, Scattering theory with two Hilbert spaces, J. Funct. Anal. 1 (1967) 342369.

[324] T. Kato, Smooth operators and commutators, Studia Mathematica 31 (1968) 535546. 
[325] T. Kato, Some results on potential scattering, in Proc. Int. Conf. Functional Analysis and Related Topics, Tokyo, 1969 (University of Tokyo Press, Tokyo, 1970), pp. 206-215.

[326] T. Kato, Scattering theory and perturbation of continuous spectra, Actes Congr. Int. Math. 1 (1971) 135-140.

[327] T. Kato, Schrödinger operators with singular potentials, Israel J. Math. 13 (1972) $135-148$.

[328] T. Kato, A remark to the preceding paper by Chernoff, J. Funct. Anal. 12 (1973) 415-417.

[329] T. Kato, Continuity of the map $S \mapsto|S|$ for linear operators, Proc. Japan Acad. 49 (1973) 157-160.

[330] T. Kato, A second look at the essential self-adjointness of the Schrödinger operators, in Physical Reality and Mathematical Description, eds. C. Enz and J. Mehra (Reidel, Dordrecht, 1974), pp. 193-201.

[331] T. Kato, On the Trotter-Lie product formula, Proc. Japan Acad. 50 (1974) 694-698.

[332] T. Kato, Perturbation Theory for Linear Operators, Grundlehren der Mathematischen Wissenschaften, 2nd edn. Band 132 (Springer, Berlin-New York, 1976).

[333] T. Kato, Boundedness of some pseudo-differential operators, Osaka J. Math. 13 (1976) 1-9.

[334] T. Kato, Trotter's product formula for an arbitrary pair of self-adjoint contraction semigroups, in Topics in Functional Analysis, Essays Dedicated to M. G. Krein, Adv. Math. Suppl. Stud., eds. I. Gohnerg and M. Kac, Vol. 3 (1978), pp. 185-195.

[335] T. Kato, Remarks on Schrödinger operators with vector potentials, Integral Equations Operator Theory 1 (1978) 103-113.

[336] T. Kato, On some Schrödinger operators with a singular complex potential, Ann. Sc. Norm. Super. Pisa Cl. Sci. IV 5 (1978) 105-114.

[337] T. Kato, On the Cook-Kuroda criterion in scattering theory, Comm. Math. Phys. 67 (1979) 85-90.

[338] T. Kato, Remarks on the selfadjointness and related problems for differential operators, in Spectral Theory of Differential Operators (Proc. Conf., Birmingham, USA, 1981), eds. I. Knowles and R. Lewis (North-Holland, New York, 1981), pp. 253-266.

[339] T. Kato, A Short Introduction to Perturbation Theory for Linear Operators (Springer-Verlag, New York-Berlin, 1982).

[340] T. Kato, Holomorphic families of Dirac operators, Math. Z. 183 (1983) 399-406.

[341] T. Kato, Remarks on holomorphic families of Schrödinger and Dirac operators, in Proc. Conf., Birmingham/Ala. 1983, Differential Equations, eds. I. Knowles and R. Lewis (North-Holland, New York, 1984), pp. 341-352.

[342] T. Kato, Nonselfadjoint Schrödinger operators with singular first-order coefficients, Proc. Roy. Soc. Edinburgh Sect. A 96 (1984) 323-329.

[343] T. Kato, $L^{p}$-theory of Schrödinger operators with a singular potential, in Aspects of Positivity in Functional Analysis, eds. R. Nagel, U. Schlotterbeck and M. Wolff (North-Holland, New York, 1986), pp. 63-78.

[344] T. Kato and H. Fujita, On a theorem for estimating eigenvalues, J. Phys. Soc. Japan 13 (1958) 215-219.

[345] T. Kato, Manuscript (1945), Mathematical Theory of Quantum MechanicsPerturbation of Eigenvalues, Hamiltonians of Atomic and Other Systems (in Japanese), ed. S. T. Kuroda, to be published.

[346] T. Kato, H. Fujita, Y. Nakata and M. Newman, Estimation of the frequencies of thin elastic plates with free edges, J. Res. Natl. Bur. Stand. 59 (1958) 169-186. 
[347] T. Kato and K. Kodaira, On the admissible wave functions, Prog. Theore. Phys. 3 (1948) 439-440.

[348] T. Kato and S. T. Kuroda, A remark on the unitarity property of the scattering operator, Nuovo Cimento 14 (1959) 1102-1107.

[349] T. Kato and S. T. Kuroda, Theory of simple scattering and eigenfunction expansions, in Functional Analysis and Related Field, ed. F. Browder (Springer-Verlag, Berlin, 1970), pp. 99-131.

[350] T. Kato and S. T. Kuroda, The abstract theory of scattering, Rocky Mountain J. Math. 1 (1971) 127-171.

[351] T. Kato and K. Masuda, Trotter's product formula for nonlinear semigroups generated by the subdifferentials of convex functionals, J. Math. Soc. Japan 30 (1978) $169-178$.

[352] T. Kato and K. Yajima, Some examples of smooth operators and the associated smoothing effect, Rev. Math. Phys. 1 (1989) 481-496.

[353] C. E. Kenig, A. Ruiz and C. D. Sogge, Uniform Sobolev inequalities and unique continuation for second-order constant coefficient differential operators, Duke Math. J. 55 (1987) 329-347.

[354] G. B. Khosrovshahi, H. A. Levine and L. E. Payne, On the positive spectrum of Schrödinger operators with long range potentials, Trans. Amer. Math. Soc. 253 (1979) 211-228.

[355] R. Killip and B. Simon, Sum rules for Jacobi matrices and their applications to spectral theory, Ann. Math. 158 (2003) 253-321.

[356] R. Killip and B. Simon, Sum rules and spectral measures of Schrödinger operators with $L^{2}$ potentials, Ann. Math. 170 (2009) 739-782.

[357] C. W. Kilmister, George Frederick James Temple. 2 September 1901-30 January 1992, Biogr. Mem. Fellows. Royal Soc. 40 (1994) 384-400.

[358] T. Kinoshita, Ground state of the helium atom, I; II, Phys. Rev. 105 (1957) 1490$1502 ; 115$ (1959) 366-374.

[359] A. Kiselev, Absolutely continuous spectrum of one-dimensional Schrödinger operators and Jacobi matrices with slowly decreasing potentials, Comm. Math. Phys. 179 (1996) 377-400.

[360] M. Klaus, Dirac operators with several Coulomb singularities, Helv. Phys. Acta 53 (1980) 463-482.

[361] M. Klaus and B. Simon, Coupling constant thresholds in nonrelativistic quantum mechanics. I. Short-range two-body case, Ann. Phys. 130 (1980) 251-281.

[362] M. Klein and R. Seiler, Power-law corrections to the Kubo formula vanish in quantum hall systems, Comm. Math. Phys. 128 (1990) 141-160.

[363] A. Kneser, Untersuchung und asymptotische darstellung der integrale gewisser linearer differentialgleichungen bei grossen reellen werthen des arguments, J. Reine Angew. Math. 117 (1897) 72-103.

[364] K. Knopp, Theory of Functions. II. Applications and Continuation of the General Theory (Dover Publications, New York, 1947).

[365] I. Knowles, On essential self-adjointness for singular elliptic differential operators, Math. Ann. 227 (1977) 155-172.

[366] I. Knowles, On essential self-adjointness for Schrödinger operators with wildly oscillating potentials, J . Math. Anal. Appl. 66 (1978) 574-585.

[367] I. Knowles, On the existence of minimal operators for Schrödinger-type differential expressions, Math. Ann. 233 (1978) 221-227.

[368] A. Knyazev, New estimates for Ritz vectors, Math. Comp. 66 (1997) 985-995. 
[369] H. Koch and D. Tataru, Carleman estimates and unique continuation for secondorder elliptic equations with nonsmooth coefficients, Comm. Pure Appl. Math. 54 (2001) 339-360.

[370] H. Koch and D. Tataru, Carleman estimates and absence of embedded eigenvalues, Comm. Math. Phys. 267 (2006) 419-449.

[371] K. Kodaira, On ordinary differential equations of any even order and the corresponding eigenfunction expansions, Amer. J. Math. 72 (1950) 502-544.

[372] J. Konrady, Almost positive perturbations of positive selfadjoint operators, Comm. Math. Phys. 22 (1971) 295-299.

[373] S. Kotani and N. Ushiroya, One-dimensional Schrödinger operators with random decaying potentials, Comm. Math. Phys. 115 (1988) 247-266.

[374] V. Kramer, Asymptotic inverse series, Proc. Amer. Math. Soc. 7 (1956) 429-437.

[375] V. Kramer, Asymptotic perturbation series, Trans. Amer. Math. Soc. 85 (1957) 88-105.

[376] M. G. Krein, The theory of self-adjoint extensions of semi-bounded Hermitian transformations and its applications. I, Rec. Math. [Mat. Sbornik] N.S. 20 (1947) 431495.

[377] M. Krein, M. Krasnoselski and D. Milman, On the defect numbers of operators in Banach spaces and on some geometric questions, Trudy Inst. Mat. Akad. Nauk Ukrain. SSR 11 (1948) 97-112.

[378] J. Krieger, Asymptotic properties of perturbation theory, J. Math. Phys. 9 (1966) 432-435.

[379] S. T. Kuroda, An example of a scattering system in Jauch's sense, Prog. Theor. Phys. 24 (1960) 461-462.

[380] S. T. Kuroda, On a theorem of Weyl-von Neumann, Proc. Japan Acad. 34 (1958) $11-15$.

[381] S. T. Kuroda, On the existence and the unitarity property of the scattering operator, Nuovo Cimento 12 (1959) 431-454.

[382] S. T. Kuroda, Perturbation of continuous spectra by unbounded operators, I, J. Math. Soc. Japan 11 (1959) 246-262.

[383] S. T. Kuroda, Perturbation of continuous spectra by unbounded operators, II, J. Math. Soc. Japan 12 (1960) 243-257.

[384] S. T. Kuroda, Scattering theory for differential operators, I, operator theory, J. Math. Soc. Japan 25 (1973) 75-104.

[385] S. T. Kuroda, Scattering theory for differential operators, II, self-adjoint elliptic operators, J. Math. Soc. Japan 25 (1973) 222-234.

[386] S. T. Kuroda, Estimates of Kato-Temple type for $n$-dimensional spectral measures, Publ. Res. Inst. Math. Sci. 43 (2007) 505-520.

[387] H. Kurss, A limit-point criterion for nonoscillatory Sturm-Liouville differential operators, Proc. Amer. Math. Soc. 18 (1967) 445-449.

[388] T. Kurtz, Extensions of Trotter's operator semi-group approximation theorems, J. Funct. Anal. 3 (1969) 111-132.

[389] T. Kurtz, A general theorem on the convergence of operator semigroups, Trans. Amer. Math. Soc. 148 (1970) 23-32.

[390] C. Lanczos, Zur theorie des starkeffektes in hohen feldern, Z. Phys. 62 (1930) 518544; Zur verschiebung der wasserstoffterme in hohen elektrischen Feldern, Z. Phys. 65 (1930) 431-455; Zur intensitätsschwächung der spektrallinien in hohen Feldern, Z. Phys. 68 (1931) 204-232.

[391] L. Landau and E. Lifshitz, Quantum Mechanics: Non-Relativistic Theory (AddisonWesley, Reading, Massachusetts, 1958). 
[392] J. J. Landgren and P. A. Rejto (part II with M. Klaus), An application of the maximum principle to the study of essential self-adjointness of Dirac operators, I and II, J. Math. Phys. 20 (1979) 2204-2211; 21 (1980) 1210-1217.

[393] J. J. Landgren and P. A. Rejto, On a theorem of Jörgens and Chernoff concerning essential selfadjointness of Dirac operators, J. Reine Angew. Math. 322 (1981) 1-14.

[394] Y. Last and B. Simon, Eigenfunctions, transfer matrices, and absolutely continuous spectrum of one-dimensional Schrödinger operators, Invent. Math. 135 (1999) 329367.

[395] Y. Last and B. Simon, The essential spectrum of Schrödinger, Jacobi, and CMV operators, J. Anal. Math. 98 (2006) 183-220.

[396] R. Lavine, Absolute continuity of Hamiltonian operators with repulsive potentials, Proc. Amer. Math. Soc. 22 (1969) 55-60.

[397] R. Lavine, Commutators and scattering theory, I. Repulsive interactions, Comm. Math. Phys. 20 (1971) 301-323.

[398] R. Lavine, Commutators and scattering theory, II. A class of one.body problems, Indiana Univ. Math. J. 21 (1972) 643-656.

[399] R. Lavine, Completeness of the wave operators in the repulsive $N$-body problem, J. Math. Phys. 14 (1973) 376-379.

[400] R. Lavine, Absolute continuity of positive spectrum for Schrödinger operators with long range potentials, J. Funct. Anal. 12 (1973) 30-54.

[401] P. D. Lax, On Cauchy's problem for hyperbolic equations and the differentiability of solutions of elliptic equations, Comm. Pure Appl. Math. 8 (1955) 615633.

[402] P. Lax and A. Milgram, Parabolic equations, in Contributions to the Theory of Partial Differential Equations, Ann. Math. Study, Vol. 33 (Princeton, New Jersey, 1954).

[403] H. Leinfelder and C. Simader, Schrödinger operators with singular magnetic vector potentials, Math. Z. 176 (1981) 1-19.

[404] A. Lenard, The numerical range of a pair of projections, J. Funct. Anal. 10 (1972) $410-423$.

[405] N. Levinson, Criteria for the limit-point case for second order linear differential operators, Časopis Pěst. Mat. Fys. 74 (1949) 17-20.

[406] B. M. Levitan and I. S. Sargsjan, Introduction to Spectral Theory: Selfadjoint Ordinary Differential Operators, Translated from the Russian by Amiel Feinstein, Translations of Mathematical Monographs (American Mathematical Society, Providence, RI, 1975).

[407] M. Lewin, Mean-field limit of Bose systems: Rigorous results, arXiv:1510.04407.

[408] E. H. Lieb, Thomas-Fermi and related theories of atoms and molecules, Rev. Mod. Phys. 53 (1981) 603-641.

[409] E. H. Lieb, Sharp constants in the Hardy-Littlewood-Sobolev and related inequalities, Ann. Math. 118 (1983) 349-374.

[410] E. H. Lieb, Bound on the maximum negative ionization or atoms and molecules, Phys. Rev. A 29 (1984) 3018-3028.

[411] E. H. Lieb and R. Seiringer, The Stability of Matter in Quantum Mechanics (Cambridge University Press, Cambridge, 2010).

[412] E. H. Lieb, R. Seiringer, J. P. Solovej and J. Yngvason, The Mathematics of the Bose Gas and its Condensation, Oberwolfach Seminars, Vol. 34 (Birkhäuser Verlag, Basel, 2005).

[413] E. H. Lieb, I. M. Sigal, B. Simon and W. Thirring, Approximate neutrality of large- $Z$ ions, Comm. Math. Phys. 116 (1988) 635-644. 
[414] J. Lions, Équations Differentielles Operatianelles et Problèmes aux Limites (Springer-Verlag, Berlin, 1961).

[415] B. A. Lippmann and J. Schwinger, Variational principles for scattering processes, I, Phys. Rev. 79 (1950) 469-480.

[416] V. É. Ljance, Some properties of idempotent operators, Teor. Prikl. Mat. $1(1958 / 59)$ 16-22.

[417] J. J. Loeffel and A. Martin, Propriétés Analytiques des Niveaux de L'oscillateur Anharmonique et Convergence des Approximants de Padé. Cargèse Lectures in Physics, Vol. 5 (Gordon and Breach, New York, 1972), pp. 415-429.

[418] J. J. Loeffel, A. Martin, B. Simon and A. Wightman, Padé approximants and the anharmonic oscillator, Phys. Lett. B 30 (1969) 656-658.

[419] K. Löwner, Über monotone Matrixfunktionen, Math. Z. 38 (1934) 177-216.

[420] E. R. Lorch, The spectrum of linear transformations, Trans. Amer. Math. Soc. 52 (1942) 238-248.

[421] J. Magnen and R. Sénéor, Phase space cell expansion and Borel summability for the Euclidean $\varphi_{3}^{4}$ theory, Comm. Math. Phys. 56 (1977) 237-276.

[422] J. Magnen and R. Sénéor, Yukawa quantum field theory in three dimensions (Y3), in Third Int. Conf. Collective Phenomena, Moscow, 1978 (New York Academy of Sciences, New York, 1980), pp. 13-43.

[423] M. Măntoiu, $C^{*}$-algebras, dynamical systems at infinity and the essential spectrum of generalized Schrödinger operators, J. Reine Angew. Math. 550 (2002) 211-229.

[424] M. Martin and M. Putinar, Lectures on Hyponormal Operators (Birkhäuser Verlag, Basel, 1989).

[425] A. Martinez, An Introduction to Semiclassical and Microlocal Analysis (SpringerVerlag, New York, 2002).

[426] A. Martinez, T. Ramond and J. Sjöstrand, Resonances for nonanalytic potentials, Anal. PDE 2 (2009) 29-60.

[427] J. McLeod, Spectral concentration I, The one-dimensional Schrödinger operator, in Perturbation Theory and its Applications in Quantum Mechanics, ed. C. H. Wilcox (John Wiley \& Sons, New York, 1966), pp. 119-127.

[428] O. Milatovic, Self-adjointness of Schrödinger-type operators with singular potentials on manifolds of bounded geometry, Electron. J. Differential Equations 64 (2003) 8.

[429] C. Møller, General properties of the characteristic matrix in the theory of elementary particles, I, Dan. Vid. Selsk. Mat.-Fys. Medd. 23 (1945) 1-48.

[430] J. Morgan, Schrödinger operators whose potentials have separated singularities, J. Opeartor Theory 1 (1979) 109-115.

[431] E. Mourre, Absence of singular continuous spectrum for certain self-adjoint operators, Comm. Math. Phys. 78 (1981) 391-408.

[432] C. Müller, On the behavior of the solutions of the differential equation $\Delta U=$ $F(x, U)$ in the neighborhood of a point, Comm. Pure Appl. Math. 7 (1954) 505515 .

[433] M. Murata, Asymptotic expansions in time for solutions of Schrödinger-type equations, J. Funct. Anal. 49 (1982) 10-56.

[434] S. N. Naboko, On the dense point spectrum of Schrödinger and Dirac operators, Teor. Mat. Fiz. 68 (1986) 18-28 [Theor. Math. Phys. 68 (1986) 646653.

[435] M. Nagumo, Einige analytische Untersuchungen in linearen, metrischen Ringen, Japan J. Math. 13 (1936) 61-80.

[436] B. Sz.-Nagy, Hungarian version of 437, Mat. Természettudományi Értesitö 61 (1942) 755-774. 
[437] B. Sz.-Nagy, Perturbations des transformations autoadjointes dans l'espace de Hilbert, Comment. Math. Helv. 19 (1947) 347-366.

[438] B. Sz.-Nagy, Perturbations des transformations linéaires fermées, Acta Sci. Math. (Szeged) 14 (1951) 125-137.

[439] S. Nakamura, Shape resonances for distortion analytic Schrödinger operators, Comm. Partial Differential Equations 14 (1989) 1385-1419.

[440] S. Nakamura, Distortion analyticity for two-body Schrödinger operators, Ann. Inst. H. Poincaré Phys. Théor. 53 (1990) 149-157.

[441] E. Nelson, Analytic vectors, Ann. Math. 70 (1959) 572-615.

[442] E. Nelson, Interaction of nonrelativistic particles with a quantized scalar field, J. Math. Phys. 5 (1964) 1190-1197.

[443] E. Nelson, Feynman integrals and the Schrödinger equation, J. Math. Phys. 5 (1964) $332-343$.

[444] E. Nelson, A quartic interaction in two dimensions, in Mathematical Theory of Elementary Particles, eds. R. Goodman and I. Segal (M.I.T. Press, Cambridge, MA, 1966), pp. 69-73.

[445] E. Nelson, Time-ordered operator products of sharp-time quadratic forms, J. Funct. Anal. 11 (1972) 211-219.

[446] G. Nenciu, Distinguished self-adjoint extension for Dirac operator with potential dominated by multicenter Coulomb potentials, Helv. Phys. Acta. 50 (1977) $1-3$.

[447] G. Nenciu, Linear adiabatic theory. Exponential estimates, Comm. Math. Phys. 152 (1993) 479-496.

[448] G. Nenciu, Linear adiabatic theory: Exponential estimates and applications, in Algebraic and Geometric Methods in Mathematical Physics, eds. A. Boutet de Monvel and V. Marchenko (Kluwer Academic Publishers, Dordrecht, 1996).

[449] J. Neveu, Theorie des semi-groupes de Markov, Univ. Calif. Publ. Stat. 2 (1958) 319-394.

[450] N. Nilsson, Essential self-adjointness and the spectral resolution of Hamiltonian operators, Kungl. Fysiogr. Sällsk. i Lund Förh. 29 (1959) 1-19.

[451] F. Odeh, Note on differential operators with a purely continuous spectrum, Proc. Amer. Math. Soc. 16 (1965) 363-366.

[452] I. M. Olë̌nik, On a connection between classical and quantum-mechanical completeness of the potential at infinity on a complete Riemannian manifold, Mat. Zametki 55 (1994) 65-73 [Math. Notes 55 (1994) 380-386].

[453] J. R. Oppenheimer, Three notes on the quantum theory of aperiodic effects, Phys. Rev. 31 (1928) 66-81.

[454] J. Ouellette, Three's company, two's a crowd: Meet the Efimov effect, Scientific American Blogs (2014), https://blogs.scientificamerican.com/cocktail-partyphysics/three-8217-s-company-two-8217-s-a-crowd-meet-the-efimov-effect/.

[455] Yu. N. Ovchinnikov and I. M. Sigal, Number of bound states of three body systems and Efimov's effect, Ann. Phys. 123 (1979) 274-295.

[456] H. Padé, Sur la réprésentation approchée d'une fonction par des fractions rationelles, Ann. Sc. Ecole Norm. Sup. 9 (1892) 1-93.

[457] S. Pancharatnam, Generalized theory of interference, and its applications. Part I. Coherent pencils, Proc. Indian Acad. Sci. A 44 (1956) 247-262.

[458] A. Pazy, Semigroups of Linear Operators and Applications to Partial Differential Equations (Springer-Verlag, New York, 1983).

[459] D. B. Pearson, A generalization of Birman's trace theorem, J . Funct. Anal. 28 (1978) 182-186. 
[460] D. B. Pearson, Singular continuous measures in scattering theory, Comm. Math. Phys. 60 (1978) 13-36.

[461] C. L. Pekeris, Ground state of two-electron atoms, Phys. Rev. 112 (1958) 1649-1658.

[462] C. L. Pekeris, $1^{1} S$ and $2^{3} S$ states of helium, Phys. Rev. 115 (1959) 1216-1221.

[463] C. L. Pekeris, $1^{1} S, 2^{1} S$ and $2^{3} S$ states of $\mathrm{H}^{-}$and He, Phys. Rev. 126 (1962) 14701476 .

[464] P. Perry, Scattering Theory by the Enss Method (Harwood Academic, London, 1983).

[465] P. Perry, I. M. Sigal and B. Simon, Spectral analysis of N-body Schrödinger operators, Ann. Math. 114 (1981) 519-567.

[466] E. Picard, Sur la détermination des intégrales de certaines équations aux dérivées partielles du second ordre par leurs valeurs le long d'un contours fermé, J. Ec. Polytech. Math. 60 (1890) 89-105.

[467] A. Pietsch, Eigenvalues and s-Numbers (Cambridge University Press, Cambridge, 1987).

[468] A. Va. Povzner, On the expansion of arbitrary functions in terms of the eigenfunctions of the operator $-\Delta u+c u$, Mat. Sb. 32 (1953) 109-156 [Amer. Math. Soc. Trans. Ser. 260 (1967) 1-49].

[469] A. Va. Povzner, On expansions in functions which are solutions of a scattering problem, Dokl. Akad. Nauk SSSR 104 (1955) 360-363.

[470] C. R. Putnam, Commutation Properties of Hilbert Space Operators and Related Topics (Springer-Verlag, New York, 1967).

[471] V. S. Rabinovich, Essential spectrum of perturbed pseudodifferential operators. Applications to the Schrödinger, Klein-Gordon, and Dirac operators, Russian J. Math. Phys. 12 (2005) 62-80.

[472] J. Rauch, Local decay of scattering solutions to Schrödinger's equation, Comm. Math. Phys. 61 (1978) 149-168.

[473] J. Rauch, Perturbation theory for eigenvalues and resonances of Schrödinger Hamiltonians, J. Funct. Anal. 35 (1980) 304-315.

[474] J. Rauch and M. Reed, Two examples illustrating the differences between classical and quantum mechanics, Comm. Math. Phys. 29 (1973) 105-111.

[475] L. Rayleigh, The Theory of Sound, Vol. I (MacMillan, London, 1877); 2nd edn. (Dover Publications, New York, 1945).

[476] M. Reed and B. Simon, Methods of Modern Mathematical Physics, I: Functional Analysis (Academic Press, New York, 1972).

[477] M. Reed and B. Simon, Methods of Modern Mathematical Physics, II: Fourier Analysis, Self-Adjointness (Academic Press, New York, 1975).

[478] M. Reed and B. Simon, Methods of Modern Mathematical Physics, III: Scattering Theory (Academic Press, New York, 1979).

[479] M. Reed and B. Simon, Methods of Modern Mathematical Physics, IV: Analysis of Operators (Academic Press, New York, 1978).

[480] W. Reinhardt, Method of complex coordinates: Application to the stark effect in hydrogen, Int. J. Quantum Chem. 10 (1976) 359-367.

[481] P. Rejto, On partly gentle perturbations, I, III, J. Math. Anal. Appl. 17 (1967) 453-462; 27 (1969) 21-67.

[482] P. Rejto, Second order concentration near the binding energy of the helium Schrödinger operator, Israel J. Math. 6 (1969) 311-337.

[483] P. Rejto, Spectral concentration for the helium Schrödinger operator, Helv. Phys. Acta 43 (1970) 652-667.

[484] P. Rejto, Some potential perturbations of the Laplacian, Helv. Phys. Acta 44 (1971) 708-736. 
[485] F. Rellich, Störungstheorie der Spektralzerlegung. I-V, Math. Ann. 113 (1937) 600-619, 677-685; 116 (1939) 555-70; 117 (1940) 356-382; 118 (1942) 462-484.

[486] F. Rellich, Die zulässigen randbedingungen bei den singulären eigenwertproblemen der mathematischen physik. (Gewöhnliche differentialgleichungen zweiter ordnung), Math. Z. 49 (1944) 702-723.

[487] F. Rellich, Halbbeschränkte differentialoperatoren höherer ordnung, in Proc. Int. Congress of Mathematicians, 1954, Amsterdam, Vol. III (North-Holland Publishing Co., Amsterdam, 1956), pp. 243-250.

[488] F. Rellich, Perturbation Theory of Eigenvalue Problems (Gordon and Breach, New York, 1969).

[489] C. Remling, The absolutely continuous spectrum of one-dimensional Schrödinger operators with decaying potentials, Comm. Math. Phys. 193 (1998) 151-170.

[490] P. Renouard, Analyticité et sommabilité "de Borel" des fonctions de Schwinger du modèle de Yukawa en dimension $d=2$. I, II, Ann. Inst. H. Poincaré Sect. A 27 (1977) 237-277; A31 (1979) 235-318.

[491] R. Riddell, Spectral concentration for self-adjoint operators, Pacific J. Math. 23 (1967) 377-401.

[492] F. Riesz, Les Systèmes d'Équations à Une Infinité D'inconnues (Gauthier-Villars, Paris, 1913).

[493] F. Riesz, Über die linearen transformationen des komplexen Hilbertschen Raumes, Acta Sci. Math. Szeged 5 (1930) 23-54.

[494] D. W. Robinson, The Thermodynamic Pressure in Quantum Statistical Mechanics, Lecture Notes in Physics, Vol. 9 (Springer-Verlag, Berlin-New York, 1971) 42-74.

[495] D. W. Robinson, Hardy inequalities, Rellich inequalities and local Dirichlet forms, arXiv:1701.05629.

[496] C. A. Rogers, Two integral inequalities, J. London Math. Soc. 31 (1956) 235-238.

[497] C. A. Rogers, The number of lattice points in a set, Proc. London Math. Soc. 6 (1956) 305-320.

[498] C. A. Rogers, A single integral inequality, J. London Math. Soc. 32 (1957) 102-108.

[499] H.-W. Rohde, Über die Symmetrie elliptischer differentialoperatoren, Math. Z. 86 (1964) 21-33.

[500] H.-W. Rohde, Kriterien zur selbstadjungiertheit elliptischer differentialoperatoren I, II, Arch. Ration. Mech. Anal. 34 (1969) 188-201, 202-217.

[501] H. Rollnik, Streumaxima und gebundene Zustände, Z. Phys. 145 (1956) 639-653.

[502] L. Rosen, A $\lambda \varphi^{2 n}$ field theory without cutoffs, Comm. Math. Phys. 16 (1970) 157183.

[503] M. Rosenblum, Perturbation of the continuous spectrum and unitary equivalence, Pacific J. Math. 7 (1957) 997-1010.

[504] N. Rougerie, De Finetti theorems, mean-field limits and Bose-Einstein condensation, arXiv:1506.05263.

[505] S. N. Roze, The spectrum of a second order elliptic operator, Mat. Sb. 80 (1969) 195-209.

[506] M. B. Ruskai, Absence of discrete spectrum in highly negative ions, I, II, Comm. Math. Phys. 82 (1982) 457-469; 85 (1982) 325-327.

[507] J. Sahbani, The conjugate operator method for locally regular Hamiltonians, J. Operator Theory 38 (1997) 297-322.

[508] Y. Saito, The principle of limiting absorption for second-order differential equations with operator-valued coefficients, Pub. Res. Inst. Math. Sci. 7 (1972) 581-619.

[509] M. Schechter, Spectra of Partial Differential Operators (North Holland, Amsterdam, 1971). 
[510] M. Schechter, Scattering theory for elliptic operators of arbitrary order, Comment. Math. Helv. 49 (1974) 84-113.

[511] M. Schechter, A new criterion for scattering theory, Duke Math. J. 44 (1977) 863872 .

[512] G. Schmidt, Spectral and scattering theory for Maxwell's equations in an exterior domain, Arch. Ration. Mech. Anal. 28 (1967/1968) 284-322.

[513] U.-W. Schmincke, Essential selfadjointness of a Schrödinger operator with strongly singular potential, Math. Z. 124 (1972) 47-50.

[514] U.-W. Schmincke, Essential selfadjointness of Dirac operators with a strongly singular potential, Math. Z. 126 (1972) 71-81.

[515] U.-W. Schmincke, Distinguished selfadjoint extensions of Dirac operators, Math. Z. 129 (1972) 335-349.

[516] T. Schonbek, Decay of solutions of Schroedinger equations, Duke Math. J. 46 (1979) 203-213.

[517] T. Schonbek and Z. Zhou, Decay for solutions to the Schrödinger equations, Comm. Partial Differential Equations 22 (1997) 723-747.

[518] E. Schrödinger, Quantisierung als eigenwertproblem, IV, störungstheorie mit anwendung auf den starkeffekt der balmerlinien, Ann. Phys. 80 (1926) 437490.

[519] I. Schur, Über Potenzreihen, die im innern des einheitskreises beschränkt sind, I, II, J. Reine Angew. Math. 147 (1917) 205-232; 148 (1918) 122-145 [I. Schur methods in operator theory and signal processing, in Operator Theory: Advances and Applications, ed. I. Gohberg, Vol. 18 (Birkhäuser, Basel, 1986), pp. 31-59, 66-88].

[520] K. Schwarzschild, Zur quantenhypothese, Sitzungsber. Kön. Preuss. Akad. Wiss. 25 (1916) 548-568.

[521] D. Sears, On the solutions of a linear second order differential equation which are of integrable square, J. London Math. Soc. 24 (1949) 207-215.

[522] I. E. Segal, Construction of nonlinear local quantum processes, I, II, Ann. Math. 91 (1970) 462-481; Invent. Math. 14 (1971) 211-241.

[523] E. Seiler and B. Simon, Bounds in the Yukawa quantum field theory: Upper bound on the pressure, Hamiltonian bound and linear lower bound, Comm. Math. Phys. 45 (1975) 99-114.

[524] R. Seiringer, Hot topics in cold gases - A mathematical physics perspective, Japan J. Math. 8 (2013) 185-232.

[525] N. A. Shenk, Eigenfunction expansions and scattering theory for the wave equation in an exterior region, Arch. Ration. Mech. Anal. 21 (1966) 120-150.

[526] M. Shubin, Essential self-adjointness for semi-bounded magnetic Schrödinger operators on non-compact manifolds, J. Funct. Anal. 186 (2001) 92-116.

[527] I. M. Sigal, Geometric methods in the quantum many-body problem: Nonexistence of very negative ions, Comm. Math. Phys. 85 (1982) 309-324.

[528] I. M. Sigal, Mathematical theory of single channel systems. Analyticity of scattering matrix, Trans. Amer. Math. Soc. 270 (1982) 409-437.

[529] I. M. Sigal, How many electrons can a nucleus bind? Ann. Phys. 157 (1984) 307-320.

[530] I. M. Sigal, Complex transformation method and resonances in one-body quantum systems, Ann. Inst. H. Poincaré Phys. Théor. 41 (1984) 103-114.

[531] I. M. Sigal, Analytic properties of the scattering matrix of many particle systems, Integral Equations Operator Theory 9 (1986) 134-153.

[532] I. M. Sigal, Sharp exponential bounds on resonances states and width of resonances, Adv. Appl. Math. 9 (1988) 127-166. 
[533] I. M. Sigal, Geometric theory of Stark resonances in multielectron systems, Comm. Math. Phys. 119 (1988) 287-314.

[534] I. M. Sigal, Life-Time of Stark Resonances, in Mathematical Quantum Field Theory and Related Topics (Montreal, PQ, 1987), pp. 233-246; CMS Conf. Proc., Vol. 9 (American Mathematical Society, Providence, RI, 1988).

[535] I. M. Sigal, Stark effect in multielectron systems: Nonexistence of bound states, Comm. Math. Phys. 122 (1989) 1-22.

[536] I. M. Sigal, Renormalization group and problem of radiation, in Quantum Theory from Small to Large Scales: Lecture Notes of the Les Houches Summer School: Vol. 95, August 2010, eds. J. Fröhlich et al. (Oxford University Press, Oxford, 2012).

[537] I. M. Sigal and A. Soffer, The $N$-particle scattering problem: Asymptotic completeness for short-range systems, Ann. Math. 126 (1987) 35-108.

[538] I. M. Sigal and A. Soffer, Asymptotic completeness of $N$-particle long-range scattering, J. Amer. Math. Soc. 7 (1994) 307-334.

[539] A. G. Sigalov and I. M. Sigal, Description of the spectrum of the energy operator of quantum mechanical systems that is invariant with respect to permutations of identical particles, Theor. Math. Phys. 5 (1970) 990-1005.

[540] B. Simon, On positive eigenvalues of one-body Schrödinger operators, Comm. Pure Appl. Math. 22 (1969) 531-538.

[541] B. Simon, Coupling constant analyticity for the anharmonic oscillator, Ann. Phys. 58 (1970) $76-136$.

[542] B. Simon, On the infinitude or finiteness of the number of bound states of an $N$-body quantum system, I, Helv. Phys. Acta 43 (1970) 607-630.

[543] B. Simon, Quantum Mechanics for Hamiltonians Defined by Quadratic Forms, Princeton Series in Physics (Princeton University Press, Princeton, 1971).

[544] B. Simon, Determination of eigenvalues by divergent perturbation series, Adv. Math. 7 (1971) 240-253.

[545] B. Simon, Summability methods, the strong asymptotic condition, and unitarity in quantum field theory, Phys. Rev. Lett. 28 (1972) 1145-1146.

[546] B. Simon, Quadratic form techniques and the Balslev-Combes theorem, Comm. Math. Phys. 27 (1972) 1-9.

[547] B. Simon, Resonances in $N$-body quantum systems with dilation analytic potentials and the foundations of time-dependent perturbation theory, Ann. Math. 97 (1973) $247-274$.

[548] B. Simon, Essential self-adjointness of Schrödinger operators with positive potentials, Math. Ann. 201 (1973) 211-220.

[549] B. Simon, Essential self-adjointness of Schrödinger operators with singular potentials, Arch. Ration. Mech. Anal. 52 (1973) 44-48.

[550] B. Simon, Schrödinger operators with singular magnetic vector potentials, Math. Z. 131 (1973) 361-370.

[551] B. Simon, The $P(\Phi)_{2}$ Euclidean (Quantum) Field Theory, Princeton Series in Physics (Princeton University Press, Princeton, 1974).

[552] B. Simon, Quantum dynamics: From automorphism to Hamiltonian, in Studies in Mathematical Physics, Essays in Honor of Valentine Bargmann, eds. E. H. Lieb, B. Simon and A. S. Wightman (Princeton University Press, Princeton, 1976), pp. $327-349$.

[553] B. Simon, Universal diamagnetism of spinless Bose systems, Phys. Rev. Lett. 36 (1976) 1083-1084.

[554] B. Simon, The bound state of weakly coupled Schrödinger operators in one and two dimensions, Ann. Phys. 97 (1976) 279-288. 
[555] B. Simon, An abstract Kato's inequality for generators of positivity preserving semigroups, Indiana Univ. Math. J. 26 (1977) 1067-1073.

[556] B. Simon, Geometric methods in multiparticle quantum systems, Comm. Math. Phys. 55 (1977) 259-274.

[557] B. Simon, Scattering theory and quadratic forms: On a theorem of Schechter, Comm. Math. Phys. 53 (1977) 151-153.

[558] B. Simon, On the absorption of eigenvalues by continuous spectrum in regular perturbation problems, J. Funct. Anal. 25 (1977) 338-344.

[559] B. Simon, A canonical decomposition for quadratic forms with applications to monotone convergence theorems, J. Funct. Anal. 28 (1978) 377-385.

[560] B. Simon, Lower semicontinuity of positive quadratic forms, Proc. Roy. Soc. Edinburgh Sect. A 79 (1977/78) 267-273.

[561] B. Simon, Resonances and complex scaling: A rigorous overview, Int. J. Quantum Chem. 14 (1978) 529-542.

[562] B. Simon, Phase space analysis of simple scattering systems: Extensions of some work of Enss, Duke Math. J. 46 (1979) 119-168.

[563] B. Simon, Functional Integration and Quantum Physics, 2nd edn. (AMS Chelsea Publishing, Providence, RI, 2005); 1st edn. (Academic Press, New York, 1979).

[564] B. Simon, Trace Ideals and Their Applications, 2nd edn. (American Mathematical Society, Providence, RI, 2005); 1st edn. (Cambridge University Press, Cambridge, 1979).

[565] B. Simon, The definition of molecular resonance curves by the method of exterior complex scaling, Phys. Lett. A 71(1979) 211-214.

[566] B. Simon, Maximal and minimal Schrödinger forms, J. Operator Theory 1 (1979) $37-47$.

[567] B. Simon, Kato's inequality and the comparison of semigroups, J. Funct. Anal. 32 (1979) 97-101.

[568] B. Simon, Brownian motion, $L^{p}$ properties of Schrödinger operators and the localization of binding, J. Funct. Anal. 35 (1980) 215-229.

[569] B. Simon, Large time behavior of the $L^{p}$ norm of Schrödinger semigroups, J. Funct. Anal. 40 (1981) 66-83.

[570] B. Simon, Some Jacobi matrices with decaying potential and dense point spectrum, Comm. Math. Phys. 87 (1982) 253-258.

[571] B. Simon, Schrödinger semigroups, Bull. Amer. Math. Soc. 7 (1982) 447-526.

[572] B. Simon, Semiclassical analysis of low lying eigenvalues. I. Nondegenerate minima: Asymptotic expansions, Ann. Inst. H. Poincaré A 38 (1983) 295-308, Errata 40 (1984) 224.

[573] B. Simon, Holonomy, the quantum adiabatic theorem, and Berry's phase, Phys. Rev. Lett. 51 (1983) 2167-2170.

[574] B. Simon, Semiclassical analysis of low lying eigenvalues. II. Tunneling, Ann. Math. 120 (1984) 89-118.

[575] B. Simon, Best constants to some operator smoothness estimates, J. Funct. Anal. 107 (1992) 66-71.

[576] B. Simon, Operators with singular continuous spectrum: I. General operators, Ann. Math. 141 (1995) 131-145.

[577] B. Simon, Operators with singular continuous spectrum, VI. Graph Laplacians and Laplace-Beltrami operators, Proc. Amer. Math. Soc. 124 (1996) 1177-1182.

[578] B. Simon, Operators with singular continuous spectrum, VII. Examples with borderline time decay, Comm. Math. Phys. 176 (1996) 713-722. 
[579] B. Simon, Some Schrödinger operators with dense point spectrum, Proc. Amer. Math. Soc. 125 (1997) 203-208.

[580] B. Simon, Schrödinger operators in the twenty-first century, in Mathematical Physics 2000, eds. A. Fokas, A. Grigoryan, T. Kibble and B. Zegarlinski (Imperial College Press, London, 2001), pp. 283-288.

[581] B. Simon, Szegö's Theorem and its Descendants: Spectral Theory for $L^{2}$ Perturbations of Orthogonal Polynomials (Princeton University Press, Princeton, NJ, 2011).

[582] B. Simon, Convexity: An Analytical Viewpoint (Cambridge University Press, Cambridge, 2011).

[583] B. Simon, A Comprehensive Course in Analysis, Part 1: Real Analysis (American Mathematical Society, Providence, RI, 2015).

[584] B. Simon, A Comprehensive Course in Analysis, Part 2A: Basic Complex Analysis (American Mathematical Society, Providence, RI, 2015).

[585] B. Simon, A Comprehensive Course in Analysis, Part 2B: Advanced Complex Analysis (American Mathematical Society, Providence, RI, 2015).

[586] B. Simon, A Comprehensive Course in Analysis, Part 3: Harmonic Analysis (American Mathematical Society, Providence, RI, 2015).

[587] B. Simon, A Comprehensive Course in Analysis, Part 4: Operator Theory (American Mathematical Society, Providence, RI, 2015).

[588] B. Simon, Unitaries permuting two orthogonal projections, to appear in Linear Algebra Appl.

[589] B. Simon and R. Høegh-Krohn, Hypercontractive semi-groups and two dimensional self-coupled Bose fields, J. Funct. Anal. 9 (1972) 121-180.

[590] J. Sjöstrand, Lectures on Resonances, Unpublished, http://sjostrand.perso.math. cnrs.fr/Coursgbg.pdf.

[591] E. Skibsted and X. P. Wang, 2-body threshold spectral analysis, the critical case, J. Func. Anal. 260 (2011) 1766-1794.

[592] A. V. Sobolev, The Efimov effect discrete spectrum asymptotics, Comm. Math. Phys. 156 (1993) 101-126.

[593] M. Sova, Problème de Cauchy pour équations hyperboliques opérationelles a coefficients constants non-bornés, Ann. Sc. Norm. Sup. Pisa 22 (1968) 67-100.

[594] G. Stampacchia, Le problème de Dirichlet pour les équations elliptiques du second ordre à coefficients discontinus, Ann. Inst. Fourier 15 (1965) 189-258.

[595] E. M. Stein and G. Weiss, Fractional integrals in $n$-dimensional Euclidean space, J. Math. Mech. 7 (1958) 503-514.

[596] H. Stetkaer-Hansen, A generalization of a theorem of Wienholtz concerning essential selfadjointness of singular elliptic operators, Math. Scand. 19 (1966) 108-112.

[597] T. Stieltjes, Recherches sur les fractions continues, Ann. Fac. Sci. Toulouse Math. (6) 8 (1894) J76-J122; 9 (1895) A5-A47.

[598] F. H. Stillinger, Ground-state energy of two-electron atoms, J. Chem. Phys. 45 (1966) 3623-3631.

[599] G. Stolz and B. Simon, Operators with singular continuous spectrum, V. Sparse potentials, Proc. Amer. Math. Soc. 124 (1996) 2073-2080.

[600] M. Stone, Linear Transformations in Hilbert Space, Reprint of the 1932 original, Vol. 15 (American Mathematical Society Colloquium Publications, American Mathematical Society, Providence, RI, 1990).

[601] F. Stummel, Singuläre elliptische Differential-operatoren in Hilbertschen Räumen, Math. Ann. 132 (1956) 150-176.

[602] D. Szyld, The many proofs of an identity on the norm of oblique projections, Numer. Algorithms 42 (2006) 309-323. 
[603] M. Takesaki, Theory of Operator Algebras I (Springer-Verlag, New York, 1979).

[604] H. Tamura, The Efimov effect of three-body Schrödinger operators, J. Funct. Anal. 95 (1991) 433-459.

[605] H. Tamura, The Efimov effect of three-body Schrödinger operators: Asymptotics for the number of negative eigenvalues, Nagoya Math. J. 130 (1993) 55-83.

[606] A. E. Taylor, Analysis in complex Banach spaces, Bull. Amer. Math. Soc. 49 (1943) 652-669.

[607] G. Temple, The theory of Rayleigh's principle as applied to continuous systems, Proc. Roy. Soc. A 119 (1928) 276-293.

[608] G. Temple, The computation of characteristic numbers and characteristic functions, Proc. Lond. Math. Soc. (2) 29 (1928) 257-280.

[609] G. Temple, The accuracy of Rayleigh's method of calculating the natural frequencies of vibrating systems, Proc. Roy. Soc. London. Ser. A. 211 (1952) 204-224.

[610] G. Temple, An elementary proof of Kato's lemma, Mathematika 2 (1955) 39-41.

[611] S. Teufel, A note on the adiabatic theorem without gap condition, Lett. Math. Phys. 58 (2001) 261-266.

[612] S. Teufel, Adiabatic Perturbation Theory in Quantum Dynamics, Lecture Notes in Mathematics, Vol. 1821 (Springer-Verlag, Berlin, 2003).

[613] W. Thirring, Quantum Mathematical Physics. Atoms, Molecules and Large Systems, 2nd edn. Translated from the 1979 and 1980 German originals by eds. M. Evans, Harrell II (Springer-Verlag, Berlin, 2002).

[614] D. W. Thoe, Spectral theory for the wave equation with a potential term, Arch. Ration. Mech. Anal. 22 (1966) 364-406.

[615] D. W. Thoe, Eigenfunction expansions associated with Schroedinger operators in $R_{n}, n 4$, Arch. Ration. Mech. Anal. 26 (1967) 335-356.

[616] D. J. Thouless, M. Kohmoto, M. P. Nightingale and M. den Nijs, Quantized hall conductance in a two-dimensional periodic potential, Phys. Rev. Lett. 49 (1982) 405-408.

[617] E. Titchmarsh, Some theorems on perturbation theory, I-V, Proc. Roy. Soc. A200 (1949) 34-46; A201 (1950) 473-479; A207 (1951) 321-328; A210 (1951) 30-47; J. Anal. Math. 4 (1954-1956) 187-208.

[618] E. Titchmarsh, Eigenfunction expansions associated with partial differential equations, V, Proc. London Math Soc. 5 (1955) 1-21.

[619] E. Titchmarsh, Eigenfunction Expansions Associated with Second Order Differential Equations, Part I, 2nd edn. (Oxford University Press, Oxford, 1962).

[620] E. Titchmarsh, Eigenfunction Expansions Associated with Second Order Differential Equations, Part II (Oxford University Press, Oxford, 1958).

[621] H. Trotter, Approximation of semi-groups of operators, Pacific J. Math. 8 (1958) 887-919.

[622] H. F. Trotter, On the product of semi-groups of operators, Proc. Amer. Math. Soc. 10 (1959) 545-551.

[623] N. Trudinger, Linear elliptic operators with measurable coefficients, Ann. Sc. Norm. Sup. Pisa Sci. Fis. Mat. 27 (1973) 255-308.

[624] R. Turner, A note on eigenvalues of normal transformations, Proc. Amer. Math. Soc. 20 (1969) 30-34.

[625] J. Uchiyama, Finiteness of the number of discrete eigenvalues of the Schrödinger operator for a three particle system, Publ. Res. Inst. Math. Sci. 5 (1969) 51-63.

[626] A. F. Vakulenko, A variant of commutator estimates in spectral theory, Kraev. Zadachi Mat. Fiz. i Smezhn. Vopr. Teor. Funktsii 19 (1987) 29-36 [J. Soviet Math. 49 (1990) 1136-1139]. 
[627] K. Veselić, On spectral concentration for some classes of selfadjoint operators, Glasnik Mat. Ser. III 4 (1969) 213-229.

[628] C. van Winter, Theory of finite systems of particles. I. The Green function, Mat.Fys. Skr. Dan. Vid. Selsk. 2(8) (1964), 1-60.

[629] J. von Neumann, Allgemeine eigenwerttheorie hermitescher funktionaloperatoren, Math. Ann. 102 (1930) 49-131.

[630] J. von Neumann, Mathematical Foundations of Quantum Mechanics (Princeton University Press, Princeton, 1996) [German original: Mathematische Grundlagen der Quantenmechanik (1932); first English translation (1955)].

[631] J. von Neumann, Charakterisierung des Spektrums eines Integraloperators, Actualités Sci. Ind. 229 (1935) 3-20.

[632] J. von Neumann and E. Wigner, Über merkwürdige diskrete Eigenwerte, Phys. Z. 30 (1929) 465-467.

[633] S. A. Vugal'ter and G. M. Zhislin, Finiteness of a discrete spectrum of manyparticle Hamiltonians in symmetry spaces (coordinate and momentum representations), Teor. Mat. Fiz. 32 (1977) 70-87; [Theor. Math. Phys. 32 (1977) 602-614].

[634] S. Wallach, On the location of spectra of differential equations, Amer. J. Math. 70 (1948) 833-841.

[635] J. Walter, Symmetrie elliptischer differentialoperatoren I, II, Math. Z. 98 (1967) 401-406; 106 (1968) 149-152.

[636] X. P. Wang, On the existence of the N-body Efimov effect, J. Funct. Anal. 209 (2004) 137-161.

[637] X. P. Wang and Y. Wang, Existence of two-cluster threshold resonances and the N-body Efimov effect, J. Math. Phys. 46 (2005) 112106.

[638] G. Watson, A theory of asymptotic series, Philos. Trans. Roy. Soc. London Ser. A 211 (1912) 279-313.

[639] J. Weidmann, The virial theorem and its application to the spectral theory of Schrödinger operators, Bull. Amer. Math. Soc. 73 (1967) 452-456.

[640] J. Weidmann, Oszillationsmethoden für systeme gewöhnlicher differentialgleichungen, Math. Z. 119 (1971) 349-373.

[641] V. Weisskopf and E. P. Wigner, Berechnung der natürlichen Linienbreite auf Grund der Diracschen Lichttheorie, Z. Phys. 63 (1930) 54-73.

[642] H. Weyl, Über gewöhnliche lineare differentialgleichungen mit singulären Stellen und ihre Eigenfunktionen I, II, Gött. Nachr. 1909 (1909) 37-63; 1910 (1910) 442-467.

[643] H. Weyl, Über beschränkte quadratische Formen, deren Differenz vollstetig ist, Palermo Rend. 27 (1909) 373-392, 402.

[644] H. Weyl, Über gewöhnliche differentialgleichungen mit singularitäten und die zugehörigen entwicklungen willkürlicher funktionen, Math. Ann. 68 (1910) 220269.

[645] H. Weyl, The Theory of Groups and Quantum Mechanics (Dover Publications, Inc., New York, 1950) [German original: Gruppentheorie und Quantenmechanik (1928)].

[646] J. A. Wheeler, On the mathematical description of light nuclei by the method of resonating group structure, Phys. Rev. 52 (1937) 1107-1122.

[647] E. Wienholtz, Halbbeschränkte partielle differentialoperatoren zweiter ordnung vom elliptischen typus, Math. Ann. 135 (1958) 50-80.

[648] E. Wienholtz, Bemerkungen über elliptische differentialoperatoren, Arch. Math. 10 (1959) 126-133.

[649] C. H. Wilcox, Wave operators and asymptotic solutions of wave propagation problems of classical physics, Arch. Ration. Mech. Anal. 22 (1966) 37-78. 
[650] A. Wintner, On the normalization of characteristic differentials in continuous spectra, Phys. Rev. 72 (1947) 516-517.

[651] E. Witten, Supersymmetry and Morse theory, J. Differential Geom. 17 (1982) 661692 .

[652] N. Wolchover, Physicists prove surprising rule of threes, Quanta Mag. (2014), https://www.quantamagazine.org/in-efimov-state-physicists-find-a- surprising-ruleof-threes-20140527.

[653] F. Wolf, Analytic perturbation of operators in Banach spaces, Math. Ann. 124 (1952) 317-333.

[654] R. Wüst, Generalizations of Rellich's theorem on perturbations of (essentially) selfadjoint operators, Math. Z. 119 (1971) 276-280.

[655] R. Wüst, A convergence theorem for self-adjoint operators applicable to Dirac operators with cut-off potentials, Math. Z. 131 (1973) 339-349.

[656] R. Wüst, Distinguished self-adjoint extensions of Dirac operators constructed by means of cut-off potentials, Math. Z. 141 (1975) 93-98.

[657] R. Wüst, Dirac operators with strongly singular potentials, Math. Z. 152 (1977) 259-271.

[658] Y. Wang, H. Du and Y. Dou, On the index of Fredholm pairs of idempotents, Acta Math. Sin. (Engl. Ser.) 25 (2009) 679-686.

[659] D. R. Yafaev, On the theory of the discrete spectrum of the three-particle Schrödinger operator, Mat. Sb. 94 (1974) 567-593 [Math. USSR-Sb. 23 (1974) 535559].

[660] D. R. Yafaev, The discrete spectrum of the three-particle Schrödinger operator, Dokl. Akad. Nauk SSSR 206 (1972) 68-70.

[661] D. R. Yafaev, The point spectrum in the quantum mechanical problem of many particles, Izv. Akad. Nauk SSSR Ser. Mat. 40 (1976) 908-948 [Math. USSR-Izv. 10 (1976) 861-896].

[662] D. R. Yafaev, The virtual level of the Schrödinger equation, Mathematical questions in the theory of wave propagation, Zap. Naučn. Sem. Leningrad. Otdel. Mat. Inst. Steklov. (LOMI) 51 (1975) 203-216, 220 [J. Sov. Math. 11 (1979) 501-510].

[663] D. R. Yafaev, Mathematical Scattering Theory, General Theory (American Mathematical Society, Providence, RI, 1992).

[664] D. R. Yafaev, Radiation conditions and scattering theory for $N$-particle Hamiltonians, Comm. Math. Phys. 154 (1993) 523-554.

[665] D. Yafaev, The scattering amplitude for the Schrödinger equation with a long-range potential, Comm. Math. Phys. 191 (1998) 183-218.

[666] D. Yafaev, Sharp constants in the Hardy-Rellich inequalities, J. Funct. Anal. 168 (1999) 121-144.

[667] D. Yafaev, Scattering Theory: Some Old and New Problems, Lecture Notes in Mathematics, Vol. 1735 (Springer-Verlag, Berlin, 2000).

[668] D. R. Yafaev, Mathematical Scattering Theory, Analytic Theory (American Mathematical Society, Providence, RI, 2010).

[669] K. Yajima, The $W^{k, p}$-continuity of wave operators for Schrödinger operators, II. Positive potentials in even dimensions $m \geq 4$, in Spectral and Scattering Theory (Sanda, 1992), Lecture Notes in Pure and Applied Mathematics, ed. M. Ikawa, Vol. 161 (Dekker, New York, 1994), pp. 287-300.

[670] K. Yajima, The $W^{k, p}$-continuity of wave operators for Schrödinger operators, J. Math. Soc. Japan 47 (1995) 551-581.

[671] K. Yajima, The $W^{k, p}$-continuity of wave operators for Schrödinger operators, III. Even-dimensional cases $m \geq 4$, J. Math. Sci. Univ. Tokyo 2 (1995) 311-346. 
[672] K. Yajima, $L^{p}$-boundedness of wave operators for two-dimensional Schrödinger operators, Comm. Math. Phys. 208 (1999) 125-152.

[673] K. Yajima, Remarks on $L^{p}$-boundedness of wave operators for Schrödinger operators with threshold singularities, Doc. Math. 21 (2016) 391-443.

[674] S. Zelditch, Park City Lectures on Eigenfuntions: Geometric Analysis, in Expanded Lecture Notes from the Graduate Summer School held at the Park City Mathematical Institute (PCMI), Park City, UT, July 2013, eds. H. L. Bray et al. (American Mathematical Society, Providence, RI, 2016), pp. 11-113.

[675] Q. Zheng and X. Yao, Higher-order Kato class potentials for Schrödinger operators, Bull. Lond. Math. Soc. 41 (2009) 293-301.

[676] G. M. Zhislin, A study of the spectrum of the Schrödinger operator for a system of several particles, Tr. Moskov. Mat. Ob̌̌č. 9 (1960) 81-120.

[677] G. M. Zhislin, On the finiteness of the discrete spectrum of the energy operator of negative atomic and molecular ions, Theor. Math. Phys. 7 (1971) 571-578.

[678] M. Zworski, Distribution of poles for scattering on the real line, J. Funct. Anal. 73 (1987) 277-296.

[679] M. Zworski, Mathematical study of scattering resonances, Bull. Math. Sci. 7 (2017) $1-85$. 